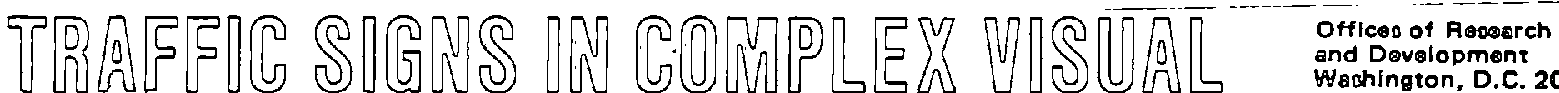
ENMVDOMNEENTS

ROPOO No.

FMUA/RO-82/102

Finol siopors

Hoy

\title{
U.S.Department
}

of Transporiation

\section{Fooleral Mighevoy}

QQobuinisirarion

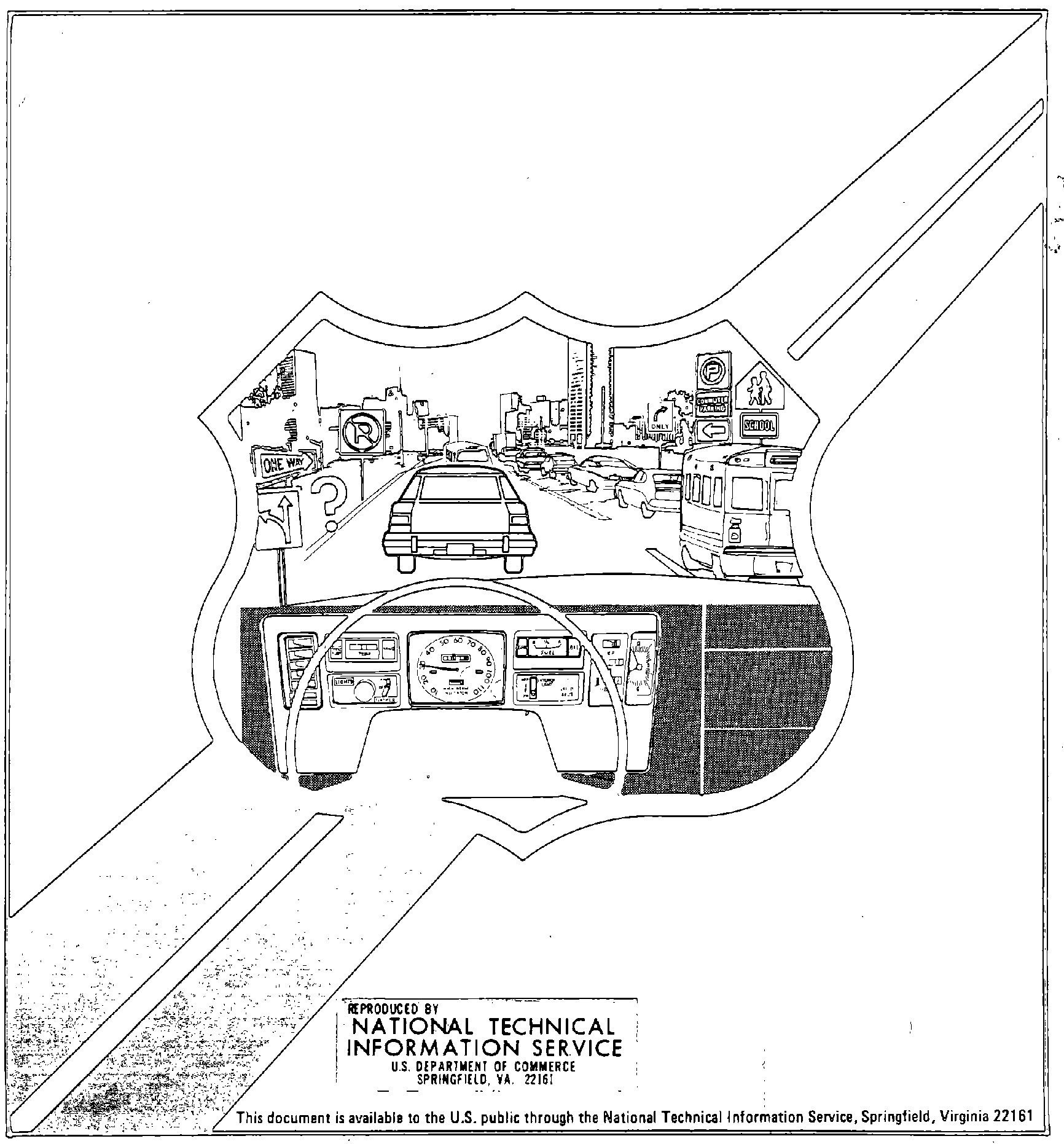




\section{FOREWORD}

This research report will be of interest to researchers and traffic engineers who are concerned with developing a performance specification for traffic signs. This study was initiated at the request of the Federal Highway Administration's Office of Traffic Operations and the National Committee on Uniform Traffic Control Devices. The report presents the results of a study titled "Performance Standards for Determining Luminous Requirements for Traffic Control Devices" undertaken by the Institute for Research. The study was conducted for the Federal Highway Administration, Offices of Research, Development, and Technology, Washington, D.C. under Contract DOT-FH-11-9646.

This report' describes a series of research studies aimed at determining the level of sign luminance required to overcome the effect of scene background complexity on detection and recognition of traffic signs at night.

Sufficient copies of the report are being distributed to provide single copies to each FHWA regional and division office and each State highway agency. Coples are also being distributed to the National Committee on Uniform Traffic Control Devices, Technical Committee on Signs, and the Transportation Research Board's Committees on Visibility and Traffic Control Devices.

;

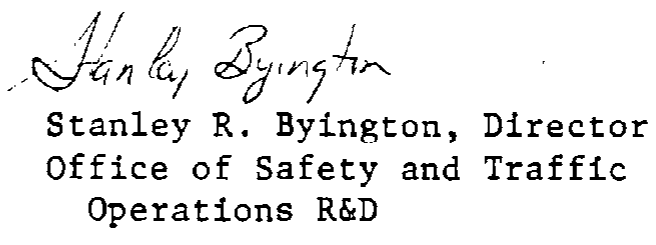

NOTICE

This document is disseminated under the sponsorship of the Department of Transportation in the interest of information exchange. The United States Government assumes no liability for its contents or use thereof.

The contents of this report reflect the views of the contractor, who 1s responsible for the accuracy of the data presented herein. The contents do not necessarily reflect the official policy of the Department of Transportation.

This report does not constitute a standard, specification, or regulation.

The United States Government does not endorse products or manufacturers. Trade or manufacturers' names appear herein only because they are considered essentlal to the object of this document. 
Techoical Repert Documentetion Page

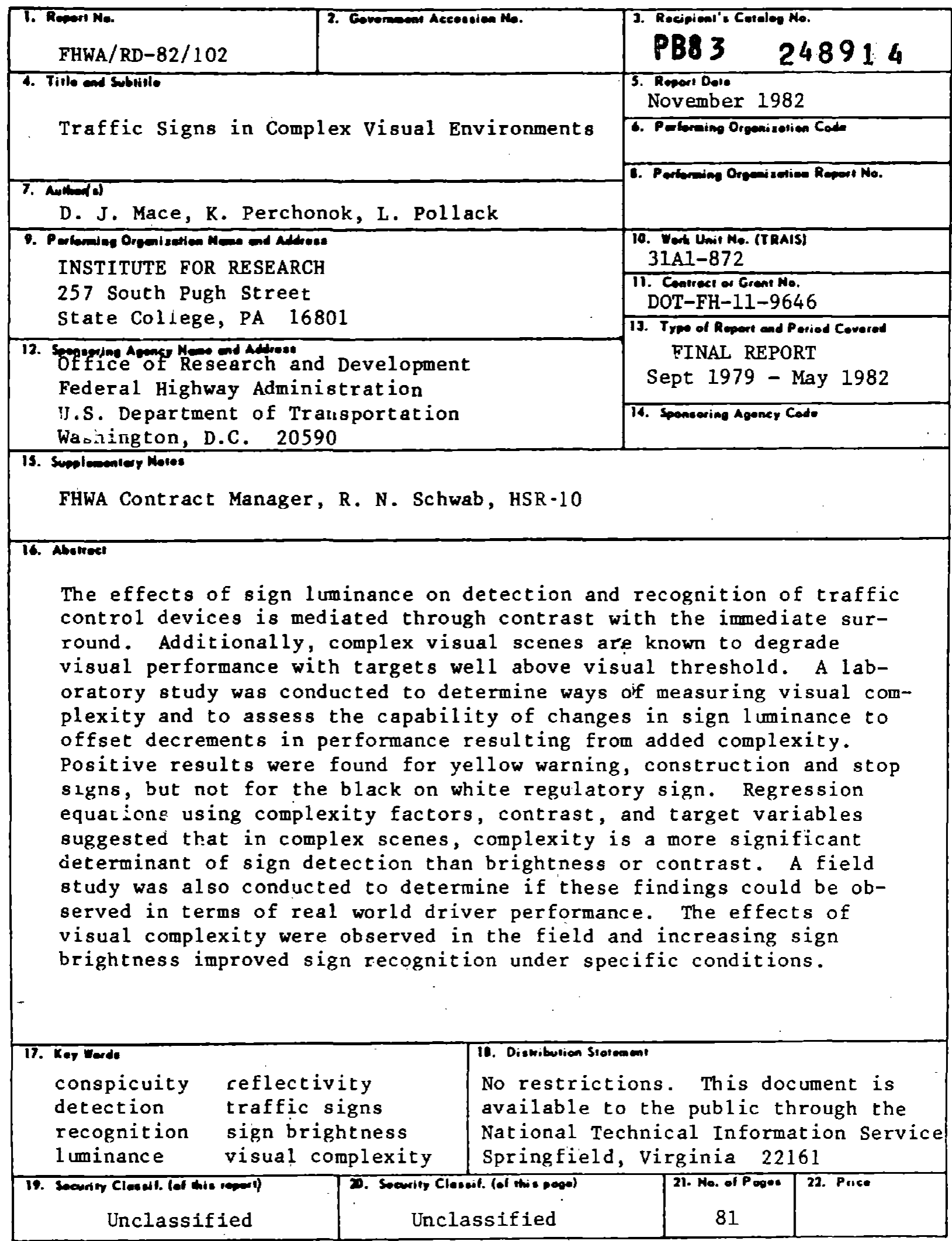

For DOT F 1700.7 (2-72) Reproduction of completod pege euthorized 


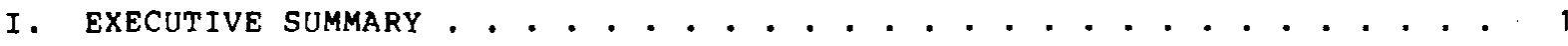

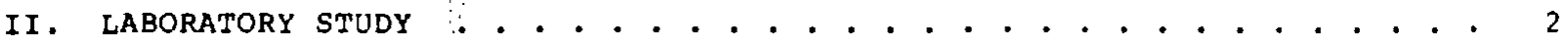

A. PURPOSE . . . . . . . . . . . . . . . . . . . . . . . . . . . . . . 2

B. EXPERIMENTAL METHOD . . . . . . . . . . . . . . . . . . . . . . . 3

1. Methodology . . . . . . . . . . . . . . . . . . . . . . 3

2. Independent Variables and the Determinants of Detection. . . 5

3. Experimental Design . . . . . . . . . . . . . . . . . . . . 7

4. A Criterion for Conspicuity . . . . . . . . . . . . . . . . . . 9

5. Procedure . . . . . . . . . . . . . . . . . . . . . . . . . . 9

6. Apparatus and stimuli . . . . . . . . . . . . . . . . . . . . 10

7. Subjects ............................ . . . 11

C. DETERMINANTS OF SIGN DETECTION . . . . . . . . . . . . . . . . . . . . 11

D. WHERE TO PUT THE SIGN? . . . . . . . . . . . . . . . . . . . . 16

1. Zero-order correlations . . . . . . . . . . . . . . 16

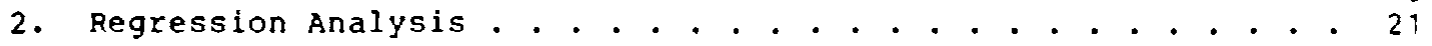

E. DISCUSSION . . . . . . . . . . . . . . . . . . . . . . . . . . . . 24

III . FIELD STUDY . . . . . . . . . . . . . . . . . . . . . . . . . 24

A. PURPOSE . . . . . . . . . . . . . . . . . . . . . . . . 24

B. Experimental method . . . . . . . . . . . . . . . . . . . . . . . 25

1. Methodology . . . . . . . . . . . . . . . . . . 25

2. Site Selection . . . . . . . . . . . . . . . . . . 25

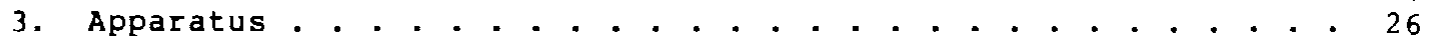

4. Data Collection procedure . . . . . . . . . . . . . . . . . . . 31

5. Subjects . . . . . . . . . . . . . . . . . . . . . . . 32

6. Independent Variables . . . . . . . . . . . . . . . . . . . . 32

7. Dependent Variables . . . . . . . . . . . . . . . . . . . . . 34

8. Experimental Design . . . . . . . . . . . . . . . . . . . 36

C. FINDINGS . . . . . . . . . . . . . . . . . . . . . . . . . . . . 36

1. Incidence of speed Reductions . . . . . . . . . . . . . . 36

2. Location of speed Reductions . . . . . . . . . . . . . . . . . 40

D. COnClusions . . . . . . . . . . . . . . . . . . . . . . . . 42

IV. SUMMARY AND RECOMMENDATIONS . . . . . . . . . . . . . . . . . . . . . . . 42

REFERENCES . . . . . . . . . . . . . . . . . . . . . . . . . . . . . . 44

APPENDIX A - Visual Dimensions of Complexity and Contrast . . . . . . . . . 46

APPENDIX B - Photometric Dimensions of Complexity, Contrast, and Sign . . . 55

APPENDIX C - SOS Experimental Instructions . . . . . . . . . . . . . . . . . 61

APPENDIX D - Means, Standard Deviations, and zero-order Correlations with Probability of Detection for all visual photometrics variables . 65

APPENDIX E - Regression Equations . . . . . . . . . . . . . . . . . 69

APPENDIX $F$ - Coded values for Subjective Dimensions of Complexity for Field Data Collection Sites . . . . . . . . . . . . . . . 77 
1. Overview of Measurements Analyzed . . . . . . . . . . . . . . . . . . 6

2. List of verbal labels for Tarqets, Chunked into categories . . . . 10

3. Distribution of Subjects by Age and Sex . . . . . . . . . . . . . . . 11

4. Multiple Correlations of Visually and photometrically netermined scene, surround, contrast, and Target Brightness Measures with Recognition Criterion... . . . . . . . . . . . . . . . . 13

5. $R^{2}$ for fach of Four Target Types Using 20 Variables from Five Groups of Measurement Categories.'. . . . . . . . . . . . . . . . 14

6. $R^{2}$ for figutions of Visual Complexity vs. Brightness (Photometric) and Contrast (Visually Measured) at Two Distances for All Signs . 15

7. Siqnificant 7ero-order Correlations of Complexity variables with probability of Recognition for DFTOUR Sign.............. . 17

8. Significant zero-order Correlations of Complexity variables with prohability of Recounition for Pedestrian Crossing siqn . . . . 18

9. Significant zero-nrder Correlations of Complexity variables with probability of Recoqnition for STOP Sign............... . 19

10. Significant zero-nrder Correlations of Complexity variables with Probability of Recoqnition for SPEF, ZONF. AHFAD Siqn. . . . . . . 19

11. Zero-order correlations of Complexity factors with Probability of netection That Are Siqnificant Across Three or Four Signs . . . . 20

12. Multiple $R^{\prime}$ 's Based upon long and short Equations with and without Contrast for four Target siqns..... . . . . . . . . . . . 22

13. F-Ratios for Main and Interactive fiffects of Distance, Complexity, and Brightness for Fach Experimental sign . . . . . . . . . . . . 24

14. Characteristics of Sites Selected for Field Data Collection . . . . 27

15. Specific Luminances and Predicted leqibility Distances of Target signs......................... 34

16. Regression fquation for visual Complexity . . . . . . . . . . . . . 35

17. Fxperimental Design for Field Data Collection: Sign luminance Conditions Balanced Across Nights and Times . . . . . . . . . . 37

18. Overall sign fffects . . . . . . . . . . . . . . . . . . 38

19. Sign fffects within Complexity levels . . . . . . . . . . . . . . . 39

20. Complexity fffects on Adjusted speed Reductions . . . . . . . . . . 39

21. Brightness Effects within Complexity Levels - Lane 1 . . . . . . . 41

22. Brightness fiffects within Complexity levels - Lane 2 . . . . . . . 4 1

23. Summary statistics for Location of Speed Reduction - lane 1. . . . 42

24. Scene variables Characteristic of the Entire Roadway Scene . . . . . 47

25. Criteria for "Point," "Medium," and "Large" Light Sources... . . 49 
27. Scene Variables within the Cone of possible Target Location . . . . 51

28. Surround variables within $1^{\circ}$ and $2^{\circ}$ Radius of Target Center..... . 52

29. Surround variables Relevant to the Target's nutside Perimeter... . 53

30. Contrast variables . . . . . . . . . . . . . . . . . . . . 54

31. Surround variables . . . . . . . . . . . . . . . . . . . . . . 55

32. Target Variables . . . . . . . . . . . . . . . . . . . 56

33. Physical Target Characteristic Measured for Target Legend varlable . 59

32. External Contrast........................ . . . . . 0

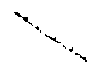

LIST OF PIGURES

1. Factorial Arrangement of Distance by Brightness by Device...... 8

2. nbservations for Each Cell of Factorial Shown in figure 1..... . 8

3. Assiqnment of Scene sets One through four to 32 Cells of

factorial Design... . . . . . . . . . . . . . . . . . . B

4. Proportion of Correct Responses for Four Levels of Distance and Two Levels of Sign Brightness . . . . . . . . . . . . . . . . . . . 12

5. Proportion of Correct Responses for Four Distances and Four Signs. . 12

6. Interaction of visual complexity and Brightness for Four Traffic signs.......................... 23

7. Target sign and Dimensions . . . . . . . . . . . . . . . . . 30

8. Photograph of shoulder-parked Car at pittsburgh (Medium Complexity) Site, Taken 100 feet upstream of the Rear of the vehicle.... . 31

9. Nighttime Photograph of the Target sign Partially Covered with Hardware Cloth......................... . 33

10. Theoretical Relationship Suggested by the Field study . . . . . . . 4

11. Four spatial Areas or zones of a Roadway scene . . . . . . . . . . . 49

12. Scaled Illustration of Aperture Location for Target Integrated Readings of pedestrian Crossing at 400 feet............ 57

13. Scaled Illustration of Aperure Locations for Target legend and Target Background Readings for Pedestrian Crossing at $400 \mathrm{feet} . .58$

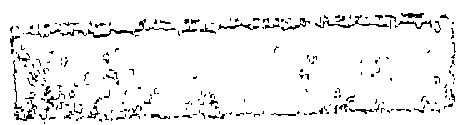




\section{EXECUTIVE SUMMARY}

The role of sign brightness and the visual complexity of the highway environment in the detection and recognition of highway traffic signs at night was studied in both laboratory and field situations. The laboratory study permitted control over a large number of highway-scenes that varied in level of visual clutter. The field study was undertaken to assess the effect of sign brightness and visual

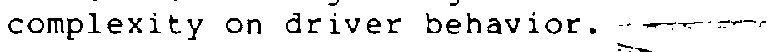

In the laboratory study, sign brightness, distance, and color-shape configuration were systematically varied within each of 80 highway scenes selected to represent the upper end of the range of visual complexity. Using $3 \times 4$ inch transparencies, "life size" images were projected for three second intervals filing a $30^{\circ}$ $x 24^{\circ}$ field of view. Subjects were required to search each stimulus and to identify the presence of other vehicles, lane lines, a curve in the road ahead, and signs of interest. Four signs were studied: Stop, Pedestrian Crossing, Detour, and speed zone Ahead. This task was intended to induce a reasonable scan pattern and task loading. The dependent variable (i.e., proportion of correct sign identifications adjusted for scene induced biases) insured a minimum level of sign recognition without requiring legibility. Visual and photometric measurements were made of the general scenes, sign surrounds, contrast, and sign brightness.

As expected, brighter signs were easier to recognize, as were closer signs. The brightness by distance interaction showed that brightness had a greater benefit at far distances than near. Not all signs were equally recognizable. The diamondshaped yellow pedestrian Crossing sign was easiest to recognize at all distances, suggesting the importance of shape discrimination in complex scenes. The black on white speed zone sign was most difficult to recognize, which might have been due to confusion with the large number of similar objects which appear in nighttime roadway scenes.

Comparisons of multiple regression equations relating stimulus characteristics to visual performance revealed that the visual measurements can be made by an observer without specialized equipment. These measurements did a better job of predicting performance than photometric measurements of the sign surround. Visual measurements were moderately superior to photometric measurements with regard to contrast. Photometric measurements, however, were superior to visual measurements of target brightness. These dif- ferences were thought to reflect substantive differences in the predictive validity of the measures and not differences in reliability of measurement. Nevertheless, the results suggest that traffic engineers should be able to evaluate complexity without specialized equipment.

of the four groups of measures -- scene, surround, contrast, and target -- scene variables predicted sign recognition performance best with surround and target brightness being less valid groups of predictors. Contrast variables were generally the poorest group although they did about as well as brightness with the SPEED ZONE sign.

Regression equations using seven measures of visual complexity (i.e. visual-measurements of scene and surround) predicted sign recognition better than sign brightness and contrast measures for all but the SPEED ZONE sign. Brightness and contrast also added little to the predictive validity of visual complexity when all measures were combined. Taken together these analyses suggest that scene complexity is somewhat more influential than sign brightness and contrast in cluttered scenes.

Correlations were examined to identify variables useful in generating hypotheses about visual complexity and as simple indicators of what traffic engineers should avoid when placing signs.

The five highest and five lowest complexity scenes within distance were identified using predicted scores based upon the regression equations for visual complexity. Analyses of the main and interactive effects of complexity, distance and brightness suggested that visual complexity produced significant differences but that the decrement in performance which occurred in high complexity scenes could (for all but the black on white sign) be offset by increasing sign brightness.

A field study was undertaken to seek confirmation of these findings in the actual performance of drivers. Three matched sites representing different levels of visual complexity were investigated. A yellow diamond warning sign with the message SPEED TRAP was deployed with three levels of luminance at each site. An unmarked car was parked upstream of the sign since a pilot test suggested this gave credibility to the sign and resulted in a greater number of observable decelerations.

The inclusion of only three sites places serious reservations on the generalizability of the findings. Nevertheless, 
the results suggest that visual complexity may degrade the recognition of traffic signs and that sign brightness may be useful in offsetting the effects of visual complexity in some situations. Sign brightness had no effect when visual complexity was either low or high. Sign brightness did however aid performance at the site with medium complexity.

Further work is needed in applying the laboratory results to the scaling of visual complexity in the real world and in determining the range of complexity which can be ameliorated by sign brightness.

\section{EABORATORY STUDY}

\section{A. PURPOSE}

A laboratory study was conducted to investigate the nighttime conspicuity of retroreflective traffic control devices in roadway environments that vary in visual complexity. Conspicuity is a perceptual construct that reflects the attention-getting value of a device within its visual field. In general, it relates measures of perceptual performance with measures of a target, the visual field, and observer uncertainty. Its effect is to promote earlier recognition of signs by offsetting decrements in probability of detection which result from driver uncertainty, poor contrast, restricted observation time, and detrimental scene or background conditions. Cole and Jenkins (1980) define a conspicuous object as one, "... that will for a given background, be seen with certainty within a short observation time, regardless of the location of the object in relation to the line of fixation." They also suggest that, "An object may have physical characteristics that render it conspicuous yet it may still be overlooked because it has no relevance to the observer. It may be noticed but immediately be shed because of its lack of relevance." This type of distinction suggested the use of sign recognition as the most appropriate criterion of whether a sign is seen and therefore conspicuous. pure detection as measured by psychophysical methods was thought to lack relevance to the driving task and to be less sensitive to variations in visual complexity.

The more practical long range goal of this research is the determination of luminance levels of retroreflective devices which will provide sufficient sign conspicuity in visual fields of different complexity. Before luminance requirements can be quantified however, it is first necessary to scale the visual complexity associated with sign placement and to delineate the interactive role of luminance for different levels of visual complexity.

The primary objective of the laboratory study was the development of a metric for visual complexity based upon those target-independent characteristics of the visual field that interfere with the detection and recognition of retroreflective traffic control devices. The detection of visual targets such as traffic signs is known to depend on characteristics of both the target and the surround. In a dynamic driving situation, detection will also depend on the time sharing effects of task loading. The visual model explored in this study attempted to use specific characteristics of the visual field and to determine their relative importance to sign detection and recognition when task loading and target variables were held constant. Unfortunately, there is very little information from previous research that specifies which characteristics are most influential in degrading perceptual performance. A few studies using traffic control devices as targets and several basic studies involving the detection of various targets within abstract visual displays have identified some potentially relevant factors.

In general, the literature suggests that detection can be considered as a function of the visual characteristics of the target and its surround. The relevant target variables include target size, shape, color, and brightness. Several studies suggest that the effect of target variables is primarily mediated through contrast with the same dimensions of the surround. For example, the attention getting value of a sign increases as the sign's size increases relative to other stimuli in the visual field (Steedman and Baker, 1960; Forbes et al., 1968a), as the sign's shape contrasts with the shape of other items surrounding the sign (Dornic and Borg, 1971), as the sign's hue contrasts with other parts of the surround (Forbes, 1968b; Saenz and Riche, 1974), and as the sign's brightness contrasts with the brightness of the immediate surround (Hanson and Dickson, 1963; Forbes et al., 1967, 1968a, 1968b; Lovie and Lovie, 1970). Of all target variables, brightness contrast has been shown to be the most important factor in determining attention getting value of signs (Hanson and Dickson, 1963; Forbes et al., 1967; Pain, 1969).

The perceptual attention-getting value of a target is not only affected by characteristics of the target but also by the characteristics of the surround within which the target is located. The 
surround variables that are most relevant include the number of noise elements, density of noise, proximity of noise to the target, and heterogeneity of the surround. In regard to the number of noise elements, a target's attentiongetting value decreases as the number of noise elements in the field increases (Baker et al., 1960; Crawford, 1962; McIntyre et al., 1970; Banks et al.. 1974; Eriksen and Eriksen, 1974; Brown and Monk, 1975; Cahill and Carter, 1976). In regard to density of noise, a target's attention-getting value: (1) decreases as the total density of noise items in the visual field increases (Green and Anderson, 1956; Smith, 1962; Smith and Thomas, 1964); and (2) decreases as the density of noise items immediately adjacent to the target increases (Monk and Brown, 1975\%. In regard to proximity of noise, a target's attention-getting

value: (1) decreases as the distance between the target and noise decreases (Banks et al., 1974; Eriksen and Eriksen, 1974; Brown and Monk, 1975; Banks et al., 1977); and (2) decreases more when noise is located peripheral to the target rather than central to the target (Mackworth, 1965; Shaw, 1969; Estes and Wolford, 1971; Wolford and Hollingsworth, 1974; Banks et al., 1977). That is, the target is less detectable when the noise is farther than the target from the center of visual field than when the noise is closer to the center of the field than the target.

One study (Jenkins, 1981) which used photographs of real, cluttered, daytime highway environments to measure the effects of background complexity, brightness, size, and contrast on detection found that contrast and size were equally important determinants of detection and that target luminance, with contrast held constant, was not significant. Background complexity was shown to have a significant and substantial effect on detection. A rank order correlation of 12 scenes ordered by subjective judgments of complexity and detection performance suggested that the subjective assessment of complexity was not adequate as a single determinant of detection. The authors conclude that while no convenient measure of complexity is available, a valid one must account for both clutter and distraction elements.
Although the literature does suggest a number of variables which might be appropriate to a measure of visual complexity, there is sufficient ambiguity in these studies to demand the empirical approach employed in the present study. First. the literature does not address the relative importance of surround characteristics and target variables, although it is generally established that brightness contrast is the most important target variable in determining attention-getting value.* Further, the literature is inconclusive regarding the size of the immediate surround and its impact versus the broader visual field. For these reasons this study considered measures of the target, target/surround, surround, and scene, although only measures of the surround and scene were considered measures of visual complexity. Further, because the effect of target variables are often mediated through relative comparisons with the surround (e.g. brightness contrast), the components of visual complexity were considered separately for different traffic signs. Finally, while the laboratory methods precluded the determination of real world sign luminance requirements, two levels of sign luminance were employed to determine whether visual complexity is susceptible to remedial treatment via improved sign brightness.

\section{B. EXPERIMENTAL METHOD}

\section{Methodology}

The selection of a methodology to accomplish the goals of this study was guided by consideration of the role of conspicuity in the detection of retroreflective traffic control devices by real-world drivers. Conspicuity, like visibility and legibility, is not an observable characteristic of a sign but a construct which relates measures of perceptual performance with measures of background, motivation, and driver uncertainty.

Given little or no uncertainty about when or where a target will appear, the perceptual performance of a motivated driver is independent of scene variables with the exception of the immediate surround. Increasing the level of scene complexity does little to degrade visual performance when the subject knows when and where the target will appear. Perceptual performance is degraded, however, when the

* This brightness contrast (which is the measure of contrast most often studied) reflects the external contrast of a sign and therefore is not considered a target variable. It is referred to in the present research as a target/surround variable to distinguish it from internal contrast (i.e. the ratio of legend to sign background) and other target variables which are more likely to be under the control of highway personnel. 
subject does not know when and/or where a visible target will appear. The amount of degradation expected increases with both the amount of uncertainty and the $v$ isual complexity of the scene. Conspicuity refers to the changes in target or surround or scene (e.g. luminance of target) which will offset the performance decrements associated with both uncertainty and complexity. Because of the asymptotic character of visibility these changes do little to affect detection frequency under conditions of low uncertainty and visual complexity.

Just as uncertainty is a prerequisite to the measurement of conspicuity, so is motivation. The effects of visual complexity cannot be measured in the complete absence of motivation any more than they can be measured in absence of uncertainty. Some level of motivation is necessary before detection can take place and uncertainty and visual complexity become relevant. The issue of conspicuity and visual complexity is just as irrelevant to the driver who is virtually asleep as it is to the driver who knows ahead of time what the sign is and where it is located. Therefore, uncertainty and motivation are not only desirable, but essential to the study of conspicuity and visual complexity.

The development of an experimental method to satisfy the research requirements attended to the manipulation or control of uncertainty, motivation, and visual complexity. Variation in visual complexity was essential to the primary goal of the experiment. To achieve the objective of scaling visual complexity, the stimuli used in this experiment had to represent a sufficient number and variety of roadway scenes to allow the identification of factors critical to influencing target recognition. This requirement mandated the use of photographic methods for the production of stimuli.

While visual complexity had to be varied, it was not necessary or even desirable to vary the attention of subjects within this experiment. The problems of visual complexity exist for the highly motivated as well as the inattentive driver and therefore lower levels of motivation are not necessary to the scaling of complexity. In fact, there are levels or types of motivation (e.g. lack of vigilance from fatigue or alcohol) in which conspicuity and/or sign brightness might not have any impact. In critical situations something extraordinary (e.g. auditory signals, advance signing, rumble strips or flashing beacons) may be necessary to gain the attention of the driver before conspicuity can have an effect. Also, methods which might be conducive to mea- suring the effects of motivation with laboratory subjects are extremely time consuming and wasteful of the experimental effort. Since subjects are naturally attentive it makes more sense to control their motivation in a way which minimizes experimental error and provides the greatest sensitivity with respect to visual complexity and the treatment effects of interest.

In the real world the attentive driver is motivated to recognize those elements in the roadway scene that are relevant at a given point in time but which vary with the specific situation and with the driver's needs. As a result, the driver is motivated to recognize, froin the universe of elements potentially relevant to driving, only a limited sample of stimuli.in order to meet his immediate information needs. This reduction in the driver's information needs is critical if the driver is not to be overwhelmed by the demands of the recognition task. Similarly, if the experimental procedure had required the subject to recognize all of the elements in each projected highway scene that are relevant to driving, the demands on the subjects would have been unrealistic and unmanageable. A more practical approach to controlling the degree of subject motivation to recognize traffic control devices in the laboratory study is one that approximates the realworld driver's level of motivation by requiring the subject to gather information about the roadway that is critical to successful driving. Toward this end, the procedure selected for the laboratory study required the subject to attend to the nature of the road, the location of other vehicles in the scene, and to a limited number of traffic control devices. Also, as is the case in the real world, a moderate degree of uncertainty about the occurrence of a particular device was maintained.

Uncertainty about sign occurrence is mediated by unfamiliarity and/ or violations of driver expectancy: The experimental stimuli made use of a large sample of photographs which were repeated a minimal number of times to insure unfamiliarity with the scenes. Within each scene the position of signs was varied among different distances and heights along the right edge of the road. Expectancies were violated to the extent that signs appeared in unconventional places (e.g. stop signs or speed control signs at signalized intersections) and tempora: uncertainty was created by random variation in the order of occurrence of the devices of interest. This methodology maintained uncertainty which is essential to measuring differential effects of visual complexity and yet controlled 
uncertainty within-definite boundaries. Devices were not located of the roadway, on the left side of the roadway, or in the roadway.

There is little doubt that an experimental method which produced less attentive drivers would result in more errors and/ or longer response latencies; however, it is by no means certain that the dimensions of scene complexity would change. similarly, a method which provided greater uncertainty by placing signs in locations with lower expectancy (e.g. in windows, cars, trees, etc.) would also produce degraded responses; however, the dimensions of visual complexity might change significantly. If the scaling of visual complexity is to be applicable to the task of detecting devices under conventional signing practices, then the dynamics of uncertainty within the experiment must direct subjects to approximate the uncertainty of real world drivers and the scanning patterns which it produces.

Since there is no practical way to control and therefore remove the effects of uncertainty, variation in uncertainty and scanning patterns becomes a major source of experimental error. This error, however, represents variation in performance which exists in the real world. Variation in uncertainty can be limited by standardized practices, but can never be entirely removed. To the extent that the variation in uncertainty induced by an experimental method approximates the variation encountered in the real world, the results with respect to such things as familiarity, expectancy, and eye scan patterns, should be generalizable.

\section{Independent Variables and the Deter-} minants of Detection

Since the role of visual complexity in conspicuity and detection is not well understood, other variables that might affect detection were incorporated into the experiment. In this way the overall importance of complexity could be assessed and the interactions with other variables documented. To this end measurements were made on visual stimuli in four categories: scene, surround, contrast, and target. Measurements in all categories were made both visually and photometrically to provide a comprehensive assessment and flexibility in the development of a complexity scale: * Alternative measures of similar concepts provide some redundancy, but also provide choices with regard to reliability, validity, and ease of measurement. The pool of variables available for analysis can be subdivided as shown in Table 1. The definition and selection of scene and surround* variables followed an iterative process which allowed for the inclusion of variables suggested by the literature as relevant to sign detection and for new variables which appeared to discriminate the stimulus scenes. By including both types of variables it was hoped that the present study would build upon previous research and extend our knowledge of the role of visual complexity in sign conspicuity and detection.

Examples of scene variables included:

- Number of Vehicles Traveling in Opposite Direction

- Visual Distractions

- Wet or Dry Pavement

- Land Use

- Number of Point Sources of Light

- Number of Traffic Signs

Examples of surround variables included:

- Number of Bright Point sources

- Number of Large Light Sources

- Uniformity of Surround

- Brightness of Surround

- Number of Different Surfaces Touching Target sign

The three independent variables manipulated, i.e. type of traffic sign, distance to sign, and brightness are discussed below. Complete definitions, and procedures for measurement of ali other variables are provided in Appendices $A$ and $B$.

In order to obtain a measure of reliability among the visual dimensions of complexity, a disagreement score was obtained for each of 15 variables which were coded independently by two staff members. $\star \star \star$ The disagreement score was given by the sum over 80 scenes of the differences in assigned values by the two raters with the result divided by the sum

* Procedures for making these measurements are documented in Appendices $A$ and $B$.

* * The surround was defined as the area outside the sign and was measured twice; once within a $1^{\circ}$ and once within a $2^{\circ}$ radius of the sign's center.

* * Upon completion of these fifteen variables (which included those listed in Table 24 of Appendix $A$ and intersection type and road type), it was decided to reduce time requirements by rating the remainder of the variables by consensus. Hence, only the fifteen variables had data available for reliability analysis. 
Table 1. Overview of Variables Analyzed

\section{Visual}

22 variables which measure uniformity and brightness of scene; number, size, and location of light sources; presence of recognizable and distracting things; general descriptions.

13 variables which measure uniformity and brightness of surround; presence of other signs; and number and size of light sources.

Proportion of perimeter darker than sign

CONTRAST Proportion of perimeter lighter than sign Proportion of perimeter equal to sign brightness.

Independent variables of SIGN distance, brightness, and sign type.

\section{Photometric}

Scene Illuminances Measured at Observer's Eye

Minimum Surround Luminance Maximum Surround Luminance Average Surround Luminance

Minimum External Contrast Maximum External Contrast Average External Contrast

Luminance of Sign Legend Luminance of Sign Background Integrated Target Luminance Internal contrast of sign of the maximum possible differences. Thus, the ratio measures amount of disagreement divided by the potential for disagreement. The maximum value observed was eight percent, for Other Than Road Detail followed by six percent for Road Surface Detail; all the other values were less than five percent with three variables showing no disagreement at all (Parked Vehicles - Right, wet/Dry Road, and Road Type). The mean of all fifteen values was 2.3 percent.

This simple analysis suggested that a high degree of agreement is quite possible among scene raters. It should be noted, however, that these raters were intimately familiar with the definitions of the variables, and that highway personnel could therefore be expected to produce somewhat less reliable ratings. None the less, serious problems would not be anticipated.

\section{Traffic Control Devices}

Only four traffic control devices were included in this study. The inclusion of so $f e w$ devices was necessitated by the need to test each device in a large number of scenes. With so few devices, the dimensions of sign variation (e.g. color, shape, and message format) could not be systematically tested. Since these dimensions varied concomitantly their effects were confounded. The limitations which this confounding could have placed on the study seemed relatively inconsequential since these device variables are restricted by the standards contained in the MUTCD. It was not the intent of this research to design devices for the purposes of optimizing nighttime conspicuity.

The four devices included in this study were:

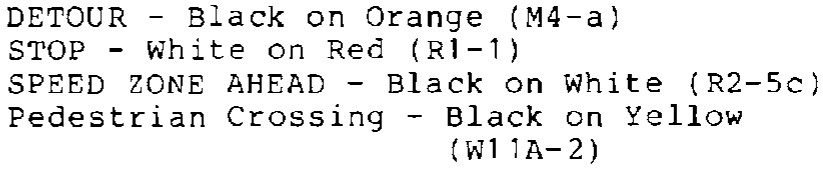

These devices (abbreviated as DTOR, STOP, SPED, and CROS throughout this report) were chosen because they represent the colors and shapes deployed most frequently in the most critical situations. The color of the orange detour sign permits generalization to a large number of construction/warning signs. The shape of the pedestrian crossing sign permits generalization to a large number of warning signs. Likewise, the shape of the black on white speed zone sign permits generalization to other regulatory signs of similar size and shape. The stop sign was chosen only for its criticality. 


\section{Device Size and Distance}

Since stimuli were two dimensional projected images, the apparent distance of each device was manipulated by the location of the device along the right edge of the road and the size of the visual angle subtended. The further away the sign was located, the smaller the image size. This variable was included to: create uncertainty necessary for the study of conspicuity and, 2) assess the effects of distance and size. Because of the importance of uncertainty in the measurement of conspicuity it was believed that four levels of this variable were essential. The choice of 250,400 , 600 , and 800 foot $(76,122,183,244 \mathrm{~m})$ distances was made to encompass the range of most recognition requirements.

\section{Device Brightness}

Since the photographic reproduction process introduces distortions in the contrast of all photometric entities, the brightness of devices was not manipulated for the purpose of estimating actual luminance requirements of signs. This objective was addressed by a later field study. Two levels of device brightness were used for the sole purpose of determining the levels of visual complexity where device brightness has the potential of improving recognition. Because of error in the photo reproduction process, variation existed within a brightness level for each sign type and distance. A reasonable effort was made to minimize the variance in brightness within each treatment cell; however because some variance existed one would expect the photometric measures of brightness (which were obtained for each stimulus) to correlate more highly with recognition than the high/low classification. The brightness of signs was held constant across distances primarily to simplify analysis and interpretation of results. The visual effect was not totally unreal. The data reported by Elstad et al. (1962) indicated that there is less than a 50 percent change in the luminance of typical traffic signs between 400 and 800 feet $(122$ and $244 \mathrm{~m})$.

\section{Experimental Design}

The design of this study was complicated by the need to assess both the effects of the independent variables which were controlled and the variables which describe visual complexity which were largeiy uncontrolled. The first of these objectives was amenable to analysis of variance while the second was suited primarily to methods of correlation and regression.
Figure 1 shows the factorial arrangement of the three independent variables. The contents of each of these cells were observations (see Figure 2) on either scenes or subjects, depending on the analysis. When the unit of observation was subjects, scores were the proportion of correct responses over 20 scenes. When the units of observations were scenes, scores were the proportion of correct responses over 40 subjects.

The complete factorial of Brightness (2) by Devices (4) by Distance (4) resulted in 32 cells. If each of these cells were crossed with each scene, either the total number of stimuli would be enormous or the number of scenes included in the study would be severely restricted. The design shown in Figure 3 represents a compromise which accomplished all of the major objectives, with 640 stimuli. The efficiency was accomplished by grouping scenes into four sets of 20 each and crossing each set of scenes with only eight of the 32 cells. As photographs of scenes were obtained it became apparent that many were suitable only for near detection and others were best used only for far detection. Therefore "Near scenes" were randomly assigned to sets 1 and 2. Likewise "Far Scenes" were randomly assigned to sets 3 . and 4 . While this randomization removed scene effects between cells involving near distances and celis involving far distances, the "self selection" of scenes for assignment to near and far distances remains a source of confounding between them.

\section{Subjects as Units of Observation}

A complete within-subjects design was employed; i.e. each $\underline{S}$ was tested on each scene within each ceIl in Figure 3. All Ss therefore were tested on each of 640 stimuli. An additional 80 stimuli were included to measure the effect of each scene without any of the four devices. These stimuli permitted the assessment and removal of scene biases from the data. The order of presentation across all 720 trials was randomly varied for each $S$ to control for possible learning and sequence effects. For purposes of analysis of variance, each cell in the three factor design contains 40 observations. Each observation is a derivation of correct responses to 20 of 80 scenes. This fractional replication of scenes between cells results in the main and interactive effects of distance being confounded with scene effects; a small price to pay for the efficiency obtained since we have little if any interest in the effects of distance. Distance was included as a variable primarily to create uncertainty concerning where a device would appear. 


\begin{tabular}{|c|c|c|c|c|c|c|c|c|}
\cline { 2 - 8 } \multicolumn{1}{c|}{} & \multicolumn{6}{c|}{ Brightness } \\
\cline { 2 - 8 } \multicolumn{1}{c|}{} & \multicolumn{4}{c|}{ Dim } & \multicolumn{5}{c|}{ Bright } \\
\hline Distance & STOP & SPED & CROS & DTOR & STOP & SPED & CROS & DTOR \\
\hline 250 & & & & & & & & \\
\hline 400 & & & & & & & & \\
\hline 600 & & & & & & & & \\
\hline 800 & & & & & & & & \\
\hline
\end{tabular}

Figure 1. Factorial Arrangement of Distance by Brightness by Device

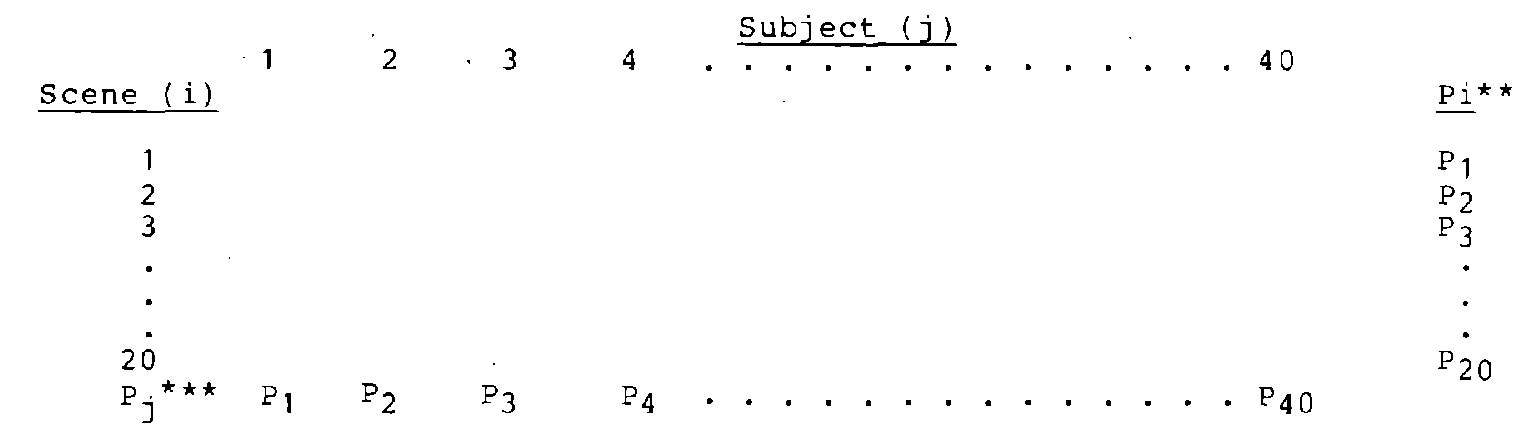

Figure 2. Observations for Each Cell of Factorial shown in Figure 1

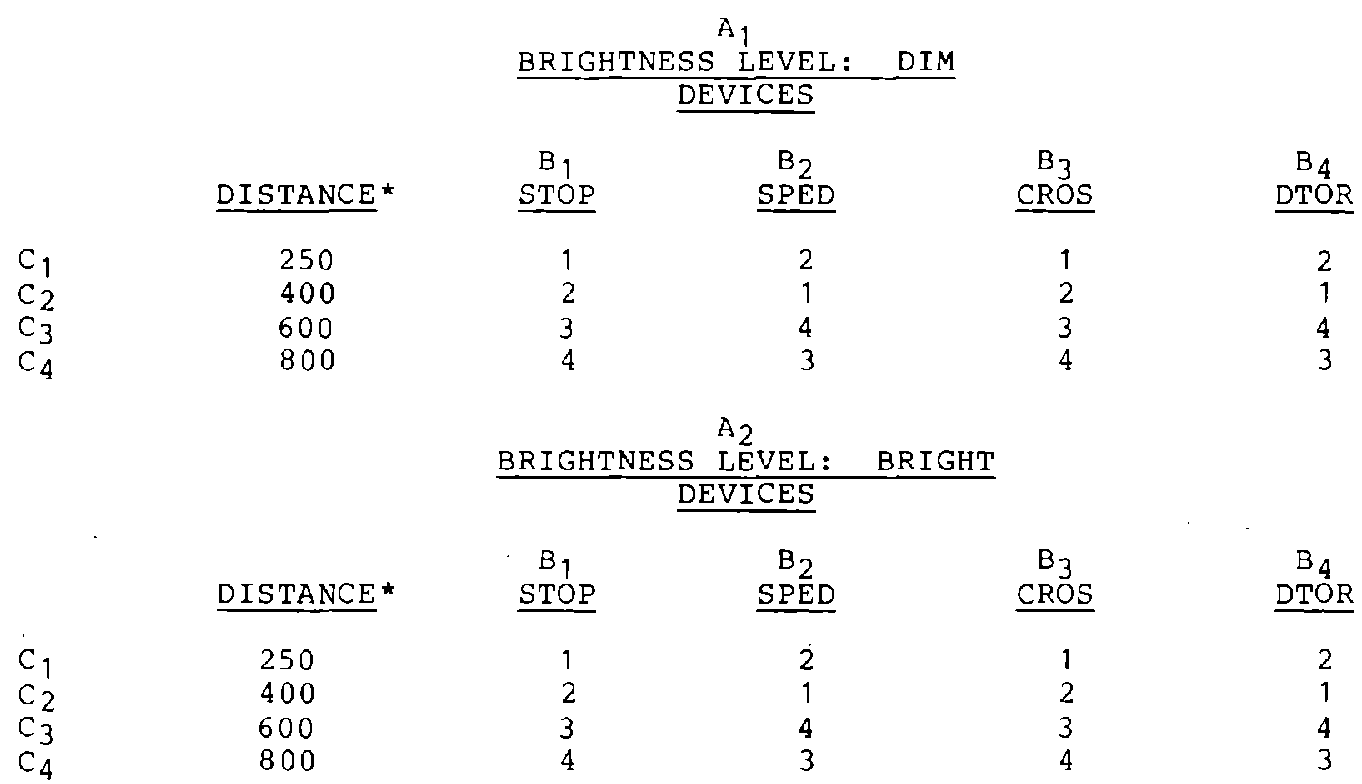

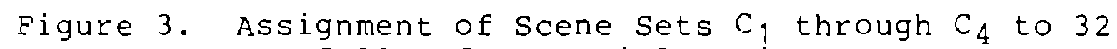
Cells of Factorial Design

* 1 foot $=.305$ meters.

* Proportions $\left(P_{j}\right)$ are based on replications over subjects to compute scene scores (rows) for regression analysis.

*** Proportions $\left(P_{j}\right)$ are based on replications over scenes to compute subject scores (columns) for AOV. 
our major interest in analyzing subjects as the units of observation was to provide an expeditious way of evaluating the main and simple interaction effects of brightness. That is, does brightness have an effect and if so do some devices benefit more than others? Also we wanted to determine whether brightness has a greater effect at some distances than at others. The lack of balance in the design with respect to scenes does not introduce any distortions into these major questions of interest.

\section{Scenes as Units of Observations}

Examination of Figure 3 indicates that each scene was tested with all devices at both levels of brightness. Also each scene in sets 1 and 2 was tested with devices at both 250 and 400 feet 176 and $122 \mathrm{~m})$ and each scene in sets 3 and 4 was tested with devices at 600 and 800 feet (183 and $244 \mathrm{~m}$ ). The total data set therefore includes 8 observations on each scene; 2 devices at 2 distances, 2 devices at another 2 distances, and all 4 of these combinations replicated at 2 brightnesses.

\section{A Criterion for Conspicuity}

The dependent variable for this study was the recognition* of signs superimposed in the stimulus scenes; the more frequently the signs were correctly identified, the greater their indicated conspicuity. Frequency of recognition appeared in the analysis in the form of proportions: for the regression analyses, the proportions were the number of correct identifications divided by the number of subjects observing each sign/scene combination; for the ANOVA, the proportions were the number of correct identifications divided by 20 , the number of scenes containing the sign for a given brightness and distance. (For the ANOVA, the arcsin transformation was applied to improve homogeneity of variances.)**

These response proportions were not thought of as estimates of real world probabilities, but only as indices by which to compare the experimental treatments.

There was a possible problem of sceneinduced biases in which scene characteristics influenced subject responses. For example, construction in a scene might be conducive to a "detour" response irrespective of the actual sign in the scene: or any naturally appearing black on white sign might increase the likelihood of a "speed zone" response. The data from the 80 scenes with no superimposed sign (the "no target" scenes) were used to correct for such biases. Specifically, for each combination of sign brightness and distance, regression analyses were used to predict scene scores (proportions) for a particular sign from the proportion of times that the sign was indicated by the subjects in the comparable no target scenes. Then the actual sign response proportion was adjusted using the predicted sign response proportion so as to yield scores which were corrected for scene-induced biases.

\section{Procedure}

The experimental procedure restricted data collection to one subject. (S) at a time, each $\underline{S}$ attending three experimental sessions scheduled on different days. During each session, which lasted about 90 minutes with two 5 -minute rest periods, an $\mathrm{S}$ responded to 240 projected stimuli so that data were collected for all 720 different stimuli after 3 sessions. The height of the $\underline{S}^{\prime} s$ chair was adjusted so that a constant eye height of 44 inches $(111.76 \mathrm{~cm})$ was maintained across S. The first session was preceded by both an initial training period and practice with the actual task. Subsequent sessions were preceded by abbreviated training and similar practice. A detailed discussion of the protocol for training, practice and the experimental trials is given in Appendix $C$. The experimental task required the subject to view nighttime road scenes and to report, using specific labels, the occurrence of any of the nine targets summarized in Table 2. Ss were instructed that each scene had one and only one of the four signs, but that any number of the other targets might appear.

During practice and the experiment Ss were shown stimuli for 3 second durations with 15 second interstimulus intervals. During the interstimulus interval, a blank image was projected to maintain a fairly constant level of dark adaptation throughout the experiment. A quiet buzzer alerted the $\mathrm{S}$ to the beginning of the next trial. The $\mathrm{s}$ was given two 5 minute breaks during each session.

* Strictly speaking, the task was one of detection followed by recognition, rather than recognition alone. This derived from the need to detect a sign and then distinguish it from both other scene elements and the other three experimentally controlled signs. Thus, only a relatively low level of recognition was required.

* The procedure suggested by winer (1978) was used to correct for extreme values of p. 
Table 2. List of Verbal Labels for Targets, chunked into Categories

\begin{tabular}{lll} 
CATEGORY & \multicolumn{1}{c}{ TARGET } & LABELS \\
Road & $\begin{array}{l}\text { road curvature } \\
\text { solid center and/or edgeline } \\
\text { dashed lane line }\end{array}$ & $\begin{array}{l}\text { curve } \\
\text { solid } \\
\text { dashed }\end{array}$ \\
Traffic & $\begin{array}{ll}\text { traffic moving in the same direction } \\
\text { traffic moving in opposite direction }\end{array}$ & same \\
Signs & $\begin{array}{l}\text { stop sign } \\
\text { pedestrian crossing } \\
\text { detour } \\
\text { speed zone ahead }\end{array}$ & stop \\
& & cross \\
& & setour \\
& speed zone
\end{tabular}

\section{Apparatus and stimuli}

The discussion of apparatus and stimuli is divided into two sections. The first section discusses the method of preparing stimuli with one of the signs embedded in each scene. The second section discusses the stimulus presentation system.

\section{Stimulus preparation}

The stimuli were composite color transparencies made from two original component transparencies: the scene and the sign. The scene transparencies were obtained in the following way.

A Ford Van was fitted with a dash level camera mount just to the right of the steering wheel. Since the van sits higher than a normal vehicle, the dashmounted camera is close to average driver eye height. After some experimentation, $35 \mathrm{~mm}$ transparencies were rejected due to poor resolution, contrast, and color rendition. The medium format Mamiya RB67 camera was selected for photographing scenes. The original scene transparencies from this camera were $6 \mathrm{~cm} \times 7 \mathrm{~cm}$ on 120 size Ektachrome Tungsten film.

After a promising scene was located, a primary marker (signs, vehicles, poles, etc.) was selected on the basis of the complexity of its background. A photograph was made at a distance from this primary marker which was determined by the speed limit at the location. Locations with posted speeds of $40 \mathrm{mph}(64.36$ $\mathrm{km} / \mathrm{hr}$ ) or more were photographed with markers 600 or 800 feet $(183$ and $244 \mathrm{~m})$ away. Locations with speeds under $40 \mathrm{mph}$ $(64.36 \mathrm{~km} / \mathrm{hr})$ were photographed with markers at 250 or 400 feet 176 and $122 \mathrm{~m})$. Looking at the scene from the camera location, a secondary marker was selected at whichever distance was not used for the primary marker.

Exact distances to the marker locations were checked using a DMI (Digital Measur- ing Instrument), an electronic "fifth wheel" device for precisely measuring the distance traveled by a wheeled vehicle. The marker descriptions recorded when the photographs were taken were used during the image combination process to achieve accurate location of the four experimental signs.

Originals of the four signs were also made with the RB 67 camera by photographing the four target devices at 250, 400, 600 , and 800 feet $(76,122,183$, and $244 \mathrm{~m}$ ) under headlight illumination on 120 Ektachrome Tungsten film.

The sign and scene components were then combined into final stimuli. Any of the signs could be inserted into any scene. Photographically prepared opaque adhesive "dots" the correct size and shape for each sign were applied to the scene original where the experimental sign was to be located. This created a "black hole" into which the sign was projected.

Using one Beseler 45 and one Beseler 23 enlarger (with colorheads), the scene and sign images were printed through a half silvered mirror and combined at the film plane of a $4 \times 5$ inch $(10.2 \mathrm{~cm} \times 12.7 \mathrm{~cm})$ Graflex film back. This arrangement provided groundglass viewing to correctly place the sign in its "black hole" in the scene. When this was achieved, a film holder containing $4 \times 5(10.2 \mathrm{~cm} \times 12.7 \mathrm{~cm})$ Ektachrome duplicating film was inserted in the Graflex film back, and the composite exposure was made. Various sign intensities could be obtained by varying the relative exposure durations of the two component orginals. The resulting composite stimuli were $31 / 4 \times 4$ inch $(8.26 \mathrm{~cm} \times 10.16 \mathrm{~cm})$ transparencies mounted in glass.

\section{Stimulus presentation}

A seven foot by ten foot glass beaded screen covering one wall of the projection room was used for viewing the 
stimulus transparencies. The projection equipment was isolated to limit sound and light contamination of the experimental situation. A Beseler Slide King Projector with a $61 / 2$ inch $(16.51 \mathrm{~cm})$ lens was situated 14 feet $(4.27 \mathrm{~m})$ from the screen, mounted behind an electronically controlled shutter. The actual size of a roadway scene on the projection screen measured 5.0 feet $(1.525 \mathrm{~m})$ vertically and 6.7 feet $(2.043 \mathrm{~m})$ horizontally. With the subject seated 11.9 feet $(3.629$ $\mathrm{m})$ from the screen $(2.1$ feet $[0.640 \mathrm{~m}]$ in front of the projector), the projected image constituted a visual field that measured $30^{\circ}$ horizontally and $24^{\circ}$ vertically.

\section{Subjects}

A total of 40 volunteer subjects participated in the study and were reimbursed for completion of all 3 sessions. They were solicited from a larger sample of subjects who had been vision tested within the previous year. The few who had not been tested were given color vision tests prior to the first session. All subjects were required to have a driver's 1 icense and to wear corrective lenses if their license required it. The sample (see Table 3 ) was evenly divided as to sex but stratified on age based upon the nighttime driving patterns reported by Compton (1980).

Inspection of the results revealed that recognition was best with the pedestrian crossing sign $(P=.89)$ and worst with the SPEED ZONE sign $(P=.57)$. The proportions for the STOP and DETOUR signs were .75 and .72 respectively. As would be expected, performance decreased from a proportion of .91 at the closest distance to .44 at the furthest distance. Bright signs resulted in .79 and dim signs resulted in .69 correct recognitions.

The distance by brightness interaction (see Figure 4 ) shows that brightness had a minimal effect at the closest distance (.90 vs. .92), a sizeable effect at 400 feet [122 m] (.80 vs. .89), and the greatest effect at 600 to 800 feet [183 and $244 \mathrm{~m}](.61$ vs. .75 and .37 vs. .52$)$. The sign type by distance interaction (see Figure 5) shows that the pedestrian crossing sign was affected least by distance and the DETOUR sign was affected the most. These results and the main effects for sign type should, however, be qualified by consideration of the fact that size is confounded with distance (as discussed above) and the response bias of subjects for each sign type.

\section{DETERMINANTS OF SIGN RECOGNITION}

Before analyzing the relative effects of scene and surround variables as determinants of sign recognition, the data were analyzed to determine the main and interactive effects of the independent variables: sign type, sign brightness, and distance. An analysis of variance of these variables revealed that all main effects and interactions (except for target $x$ brightness) were significant. For purposes of this analysis, the within-cell data were collapsed across scenes to compute a proportion of correct responses for each $\mathrm{s}$. The means reported are based on these proportions although an arcsin transformation was used to perform the statistical tests.

The responses of subjects to stimuli without signs produced the following data:

$\begin{array}{lcc}\text { Sign Type } & \begin{array}{c}\text { Number of } \\ \text { Guesses }\end{array} & \begin{array}{c}\text { Proportion of } \\ \text { Guesses }\end{array} \\ \text { STOP } & 401 & .28 \\ \text { SPEED zONE } & 911 & .65 \\ \text { DETOUR } & 169 & .12 \\ \text { Pedestrian } & 304 & .22 \\ \quad \text { Crossing } & & \end{array}$

A Chi square test indicated that there were significant differences in the response bias to the four signs ( $p<.01)$. The SPEED ZONE sign was guessed a good deal more than chance and the DETOUR sign less. of course, these proportions are overall estimates and should be applied carefully. For example, they would be more applicable to distant signs due to

Table 3. Distribution of Subjects by Age and Sex

\begin{tabular}{|c|c|c|c|c|c|}
\hline \multirow[b]{2}{*}{ Age } & \multirow[b]{2}{*}{ Group } & \multirow{2}{*}{$\begin{array}{c}\$ \text { of } \\
\text { Nighttime Driver } \\
\text { Population } \\
\end{array}$} & \multicolumn{3}{|c|}{ Sample Distribution } \\
\hline & & & Male & Female & Total \\
\hline 16 & -24 & 34 & 9 & 4 & 13 \\
\hline 25 & -34 & 26 & 5 & 6 & 11 \\
\hline 35 & -44 & 16 & 3 & 5 & 8 \\
\hline 45 & -55 & 12 & 1 & 3 & 4 \\
\hline$>$ & 55 & 12 & 2 & 2 & 4 \\
\hline
\end{tabular}




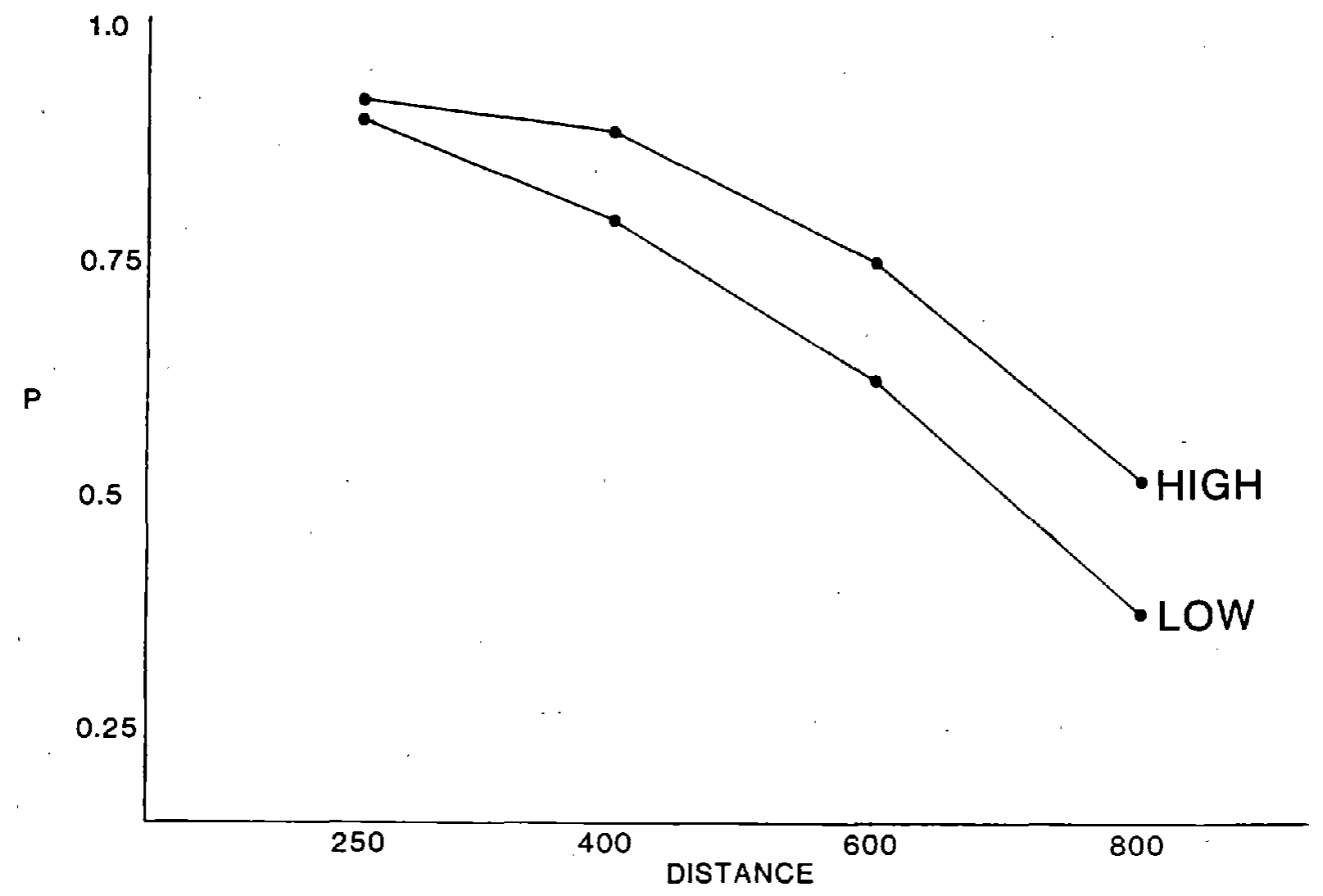

Figure 4. Proportion of Correct Responses (p) for 4 Levels of nistance and 2 Levels of Sign Brightness

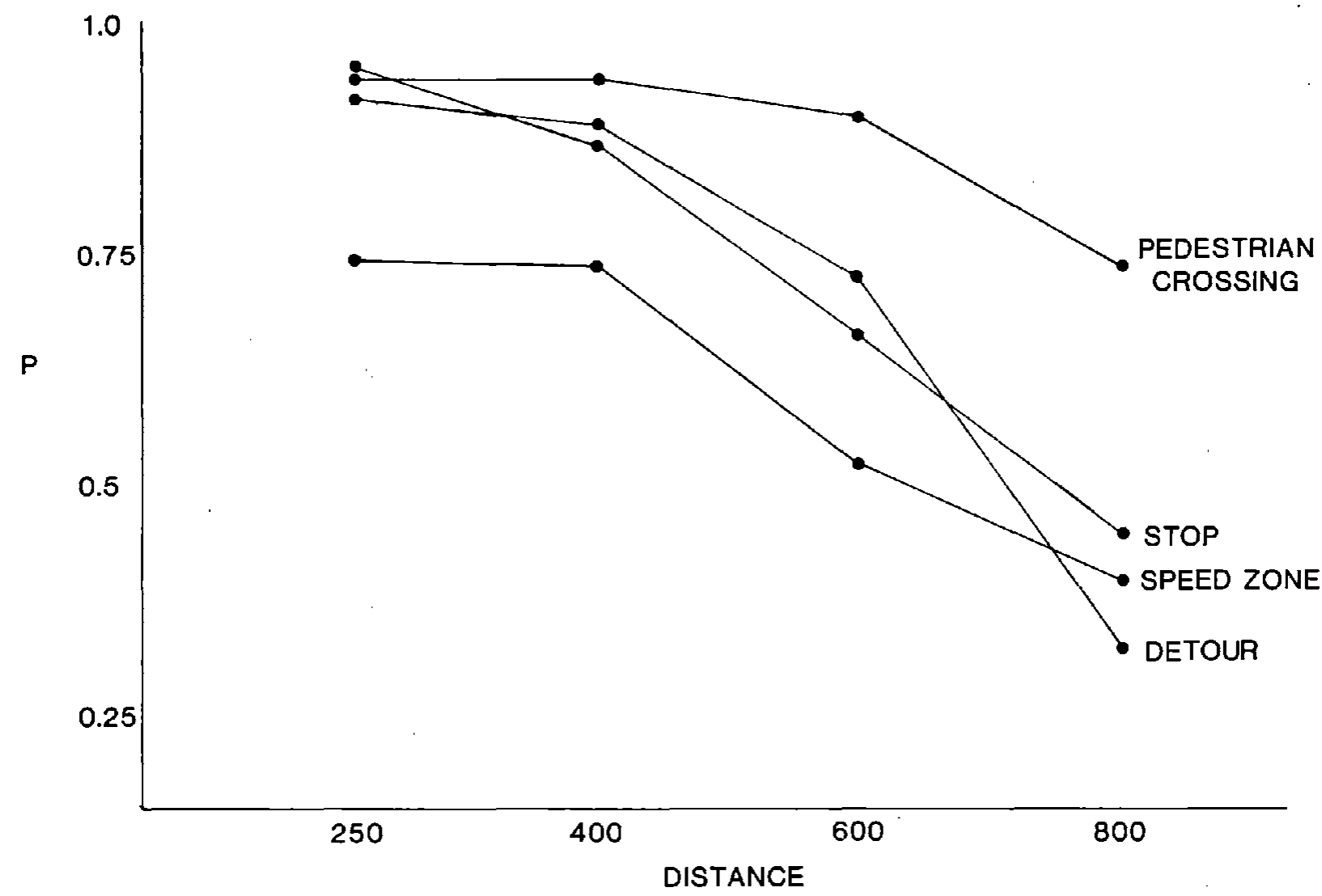

Figure 5. proportion of Correct Responses (p) for 4 Distances and 4 Signs 
the greater ambiguity of that situation. If the response bias were eliminated, one would expect the performance with the SPEED ZONE sign to be even worse than its already poor 57 percent recognition rate. Likewise, performance with the DETOUR sign should have been better than the observed 72 percent, and the negative effect of the 800 foot $(244 \mathrm{~m})$ distance on the DETOUR sign might have been reduced. *

These response biases are interestingly similar to what one might expect based upon the frequency of use of each type of sign in the real world. Black on white vertical rectangle signs are encountered most often and black on orange construction signs the least often. The biases for the SPEED ZONE sign might also result from its confusion with point sources of light. Both of these inducements for biases appear to be real and not an artifact of the experimental situation.

To evaluate the relative effectiveness of different determinants of sign recognition, regression equations were computed for each of four groups of variables obtained visually and photometrically. The multiple $\mathrm{R}^{\prime} \mathrm{s}^{\star}$ * obtained for scene, surround, contrast, and target brightness and the zero order $R^{\prime} S$ for distance and photometrically determined scene illuminance are shown in Table 4. It should be remembered that the visual and photomet$r$ ic measurements within a category were not designed to measure the same thing, only the same concept or domain. Where one correlation is significantly higher than the other it does not necessarily imply that one method of measurement is more reliable. The differences are just as likely to be attributable to differences in validity since the underlying variables were generally different in substance as well as different in the method of measurement. For example, where visual variables resulted in a higher correlation than photometric measurements, the difference is probably attributable to the fact that visual variables capitalized on the ingenuity and flexibility of the subjective process involved. Obviously the 22 different variables which measure scenes are accounting for more variance in the scene than a single measure of scene illuminance.

Inspection of Table 4 suggests that visually determined measures of the scene and surround were better predictors of recognition than were photometrically determined measures. There was not much difference in visual and photometric measurements of contrast, while photometrically determined measures of target brightness were superior to visually determined brightness.

Table 4. Multiple Correlations of Visually and Photometrically Determined Scene, surround, Contrast, and Target Brightness Measures with Recognition Criterion

\begin{tabular}{|c|c|c|c|c|c|c|c|c|}
\hline & \multicolumn{2}{|c|}{ STOP } & \multicolumn{2}{|c|}{ DTOR } & \multicolumn{2}{|c|}{ CROS } & \multicolumn{2}{|c|}{ SPED } \\
\hline & Visual & Photo & Visual & Photo & Visual & Photo & Visual & Photo \\
\hline Scene & .70 & $.06 * \star \star$ & .53 & $-.32 * \star \star$ & .66 & $-.31 * k \star$ & .72 & 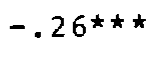 \\
\hline surround & .48 & .12 & .60 & .26 & .44 & .10 & .61 & .19 \\
\hline Contrast & .28 & .24 & .38 & .39 & .27 & .12 & .52 & .42 \\
\hline Target Brightness & .27 & .59 & .26 & .52 & .15 & .49 & .31 & .54 \\
\hline Distance $e^{\star \star \star \star}$ & -.61 & - & -.54 & - & -.52 & - & -.45 & - \\
\hline
\end{tabular}

* In order to correct for these biases, it would have been necessary to adjust subject scores. This was not possible because not all subjects gave their best guesses as directed; rather they gave no response at all thereby precluding useful estimates from the no target data.

* * Multiple R's were computed using a forward stepwise inclusion procedure. Variables were added to the equations until either all variables in a class had been used or the number of variables reached 20 . With 20 variables the predictive equation had essentially reached an asymptotic level.

$\star \star \star$ zero Order Correlation of Scene Illumination with criterion

$\star \star \star *$ Zero order correlation 
In general, the determinants of recognition in order of importance were scene, surround, target brightness, and external contrast. Although surround factors were more important than target brightness (measured photometrically) for DETOUR and SPEED ZONE signs, the reverse was true for the STOP and Pedestrian crossing signs. In any case, however, these differences were not large. Scene effects were most important for all but the DETOUR sign where the difference between the effects of scene and surround factors was small $(R=.53$ vs. .60$)$.

The zero order correlation of illuminance with the criterion revealed that brighter scenes resulted in poorer performance for all but the STOP sign where the correlation was not significant. This may be attributable to the fact that the stop sign was the darkest target used and therefore the direction of contrast in bright scenes was not unfavorable to its recognition.

The zero order correlations with distance are in Table 4 for reference. In general this variable accounted for less variance than scene variables but more variance than the surround. In absolute terms, distance/size accounted for between 20 percent (SPEED ZONE) and 36 percent (STOP) of the variance in recognition. (The variance accounted for is given by the correlation or multiple $R$ squared.)

since the determinants of recognition, i.e. scene, surround, contrast, and brightness, are not independent of each other, multiple regressions were computed to evaluate the increments in predicted variance of using surround, contrast, and target variables in addition to scene variables. The $\mathrm{R}^{2}$ for these equations is show in Table 5 .
The predicted variance for scene variables ranged from 28 percent (DETOUR) to 52 percent (SPEED 2 ONE). About half of the difference in the predicted variance between these two signs was eliminated by the inclusion of surround variables in the regression equation. It is the $\mathrm{R}^{2}$ for scene and surround which tells us the proportion of variance in recognition probability associated with scene complexity. For all signs, scene complexity (scene + surround variables) accounted for more than 50 percent of the variance in the recognition criterion.

The inclusion of contrast variables added little to the overall predictive validity; the greatest effect being a 4 percent increase from 53 to 57 percent for the DETOUR sign. The effect of adding target brightness was greater, ranging from a 4 percent increment for the pedestrian crossing sign to 10 percent for the STOP and SPEED ZONE signs. Brightness probably would have had even more importance if it had been put into the equation before contrast. The inclusion of distance had a modest effect of 7 percent and 6 percent for the DETOUR and $S T O P$ signs but no effect for the SPEED ZONE sign.

While these data provide strong support for the relative importance of scene complexity as a determinant of detection, one might still question whether or not the combined predictive validity of target size, target brightness, and external contrast might not be as great. This comparison was not possible using the data from this study because distance as manipulated confounded sign size with scenes. While the experimental design included a perfect correlation between distance and sign size, the scene selection process and the subsequent

Table 5. $\mathrm{R}^{2}$ for Each of Four Target Types using 20 variables from Five Groups of Measurement Categories

$\begin{array}{lcccr}\text { Measurement Category } & \text { DTOR } & \text { CROS } & \text { STOP } & \text { SPED } \\ \text { Scene } & .28 & .44 & .49 & .52 \\ \text { Scene + Surround } & .53 & .61 & .57 & .66 \\ \text { Scene + Surround + Contrast } & .57 & .62 & .60 & .66 \\ \begin{array}{l}\text { Scene + Surround + Contrast } \\ + \text { Brightness }\end{array} & .63 & .66 & .70 & .76 \\ \begin{array}{l}\text { Scene + Surround + Contrast } \\ + \text { Brightness* + Distance }\end{array} & .70 & .72 & .76 & .75\end{array}$

* Brightness was represented by the four photometric measurements of target brightness. 
assignment to treatment cells resulted in 62 percent of the variance in distance being predictable from the complexity measures. Therefore, if distance were used as a predictor of recognition more than half of its validity might result from its variance common to visual complexity.

An alternative analysis was to keep distance constant and compare the predictive validity of visual complexity with that of sign brightness and external contrast alone. Table 6 presents these comparisons at 400 and 600 feet $(122$ and $183 \mathrm{~m})$. Photometric measures were used for brightness and visual measures for contrast since (as indicated in Table 4) these had the highest validities.

The predictive validity $\left(R^{2}\right)$ of visual complexity (scene and surround) appears to be consistently higher at the furthest distance. These validities also appear stable across sign types, ranging from .52 to .58 at 400 feet $(122 \mathrm{~m})$ and .62 to .72 at 600 feet $(1 \mathrm{~B} 3 \mathrm{~m})$. The validities for brightness and contrast showed greater variability between signs. In only one instance (SPEED ZONE, 40 J FEET [122 m]) did brightness and contrast have a greater predictive validity than visual complexity. In all other instances, visual complexity had a greater validity. In general, the differences in validities were greatest at 600 feet $(183 \mathrm{~m})$ since (with the exception of the DETOUR sign) the validity of visual complexity increased with distance while the validity of brightness and contrast decreased or remained the same.

While the magnitude of these R's would appear to suggest that visual complexity is of overwhelming importance for most signs, the issues of reliability and generalizability must be considered. reliability of a multiple regression coefficient is affected by the size of $\mathrm{N}$ (i.e. number of observations or scenes), the number of predictor variables, and the number of variables that the predictor variables were selected from. Reliability can only be estimated when one has an a priori hypothesis to test and the variables in the regression list are preselected. Because we used an empirical approach and did not have a priori hypotheses the reliability of regression equations reported in this and the next chapter is difficult to estimate. Most of the equations for brightness and contrast used three variables and, with distance held constant, the number of observations was equal to 40 . With three variables and an $R$ of . 5 , we would be 95 percent certain the true $R$ was greater than .18 if we had preselected the variables in the equations. The equations for visual complexity are sometimes based upon seven variables and sometimes on asymptotic values using up to 20 variables. With seven variables an $R$ of .61 would be required to yield the same certainty that the true $R$ was greater than .18. While this large an $R$ was obtained, the variables were empirically determined and therefore the equations have less, but an unknown, reliability.

The reliability of other $R^{\prime} s$ reported in this and the next chapter is similarly in question. The situation is somewhat improved because the other R's in this chapter are based upon 120 observations and those in the next chapter $160 .{ }^{*}$ with the larger $N$, the visual complexity formulas would require an $R$ of about .5 using seven a priori selected variables to achieve the same reliability as the .61 Rith only 40 observations. The short formulas reported in the next chapter used six or seven variables and achieved $R^{\prime} s$ as high as .7. However, since they were not determined a priori their reliability is also in fact unknown.

Table 6. $\mathrm{R}^{2}$ for Equations of Visual Complexity vs. Brightness (Photometric) and Contrast (Visually Measured) at Two Distances for All signs

\begin{tabular}{|l|c|c|c|c|c|c|c|c|}
\hline TYPE OF SIGN & SPED & SPED & STOP & STOP & CROS & CROS & DTOR & DROR \\
\hline DISTANCE & 400 & 600 & 400 & 600 & 400 & 600 & 400 & 600 \\
\hline BRIGHT + CONTRAST & .76 & .69 & .43 & .33 & .22 & .23 & .43 & .58 \\
\hline $\begin{array}{l}\text { VISUAL COMPLEXITY } \\
\text { (7 Variables) }\end{array}$ & .68 & .72 & .52 & .62 & .58 & .69 & .63 & .64 \\
\hline
\end{tabular}

* The difference being that the observations using 800 foot (244 m) distances were omitted in this chapter because of incomplete photometric measurements. 
Obviously, additional work is needed if the data from this study are to eventually yield useful and practical results. With the zero order correlations and regression equations reported in the appendix, one should set out to define hypotheses to test about visual complexity. This may require some additional measurements of the same scenes. This analytic effort should be supplemented with cluster and factor analysis as useful techniques to define the relevant and independent dimensions of complexity while at the same time reducing the colineakity among the predictor measures. The reliability of the equations using these a priori hypotheses could then be estimated.

The analyses reported in this section provide convincing evidence for a number of things.

- Visual measurement of scene, surround, and contrast variables appears to be practical and reliable.

- with the exception of sign brightness, visual measurement resulted in more valid indicators of recognition than photometric measurements.

- Of the four categories of predictor variables studied, scene variables had the highest validity; of the combined categories studied, scene and surround (visual complexity) had the highest validity.

- The success of visual complexity as a measure of recognition must be tempered by the fact that it requires more variables to measure than the categories of contrast and brightness, and therefore may be more difficult to use.

- Highway signs are not equaliy recognizable: distance, size, and visual complexity have differential effects on each.

\section{WHERE TO PUT THE SIGN}

In the previous section, results were presented within the theoretical context of the determinants of recognition. We learned that more than half of the variance in the probability of recognition was predicted from measures of visual complexity alone. Measures of the sign, including brightness, and measures of contrast were able to add only 13 percent to the predictive validity for the STOP sign and 10,5 , and 10 percent to the predictive validity for the DETOUR, Pedestrian Crossing, and SPEED ZONE signs respectively. With the knowledge that scene and surround variables were important determinants of recognition, this chapter attempts to set forth guidelines and formulas for the optimal placement of each of the signs tested.

One perspective for providing guidelines is the identification of the most salient measures of scene and surround variables which can improve or impede recognition. This was done by examination of the significant zero-order correlations between all of variables measured and the recognition criterion. The second perspective is the quantification of the visual complexity of a specific sign placement. This was done by computing regression equations which represent the best linear combination of the predictor variables which maximize the validity of a predicted recognition score.

\section{Zero-order Correlations}

The purpose of this section is to discuss the zero order correlations of individual complexity variables with the probability of recognition for each of the four tar-. get signs. A comprehensive listing of these correlations is provided in

Appendix D. The discussion here will be limited to those variables that correlated significantly with visual performance. The level at which these relationships were considered to be significant was $p=0.05$ for a two-tailed test. That is, the correlation was judged to be significant when the magnitude of the correlation coefficient was sufficiently large that it could be expected to occur as a result of chance only one in 20 times. Given the fact that 252 coefficients were computed (i.e. 63 variables $x 4$ targets), approximately 13 correlations that were significant according to the criterion could be attributable to chance.* Because the samples were not large enough to cross validate the obtained relationships, no attempt was made to identify which correlations were chance occurrences. For this reason, caution should be exercised in drawing conclusions on the basis of any single relationship. Of course, the larger the magnitude of the coefficient, the more confidence can be placed in the reliability of the relationship. Likewise, the occurrence of a significant correlation of a given variable with performance across more than one device could be interpreted as providing some indication of a de facto relationship. In

* This estimate is a rough one in that it assumes independence among the correlation coefficients. 
discussing the simple correlations, those variables that were significantly related to the probability of recognizing a given device will be considered first, and then attention will be focused on those variables that correlated with performance across devices.

Trends among Variables within Target Type

Tables $7,8,9$, and 10 list the variables that significantly correlated with the probability of recognizing the DETOUR, Pedestrian Crossing, STOP, and SPEED ZONE signs, respectively. Each of these tables presents correlation coefficients based on the 160 stimuli containing the specified sign; as such, the data considered include all four target distances and both sign brightness conditions. These data have their greatest utility for guiding future research in visual complexity. They identify the variables which should be explored further in controlled studies. One potential practical application is their utilization as a means of quickly determining which of a series of roadway locations is likely to have the lowest complexity. While the magnitude of these correlations is not sufficient (compared to the multiple $R^{\prime} s$ ) to allow much precision in the measurement of complexity, these zero-order correlations might be used as a general guide in selecting placements that are likely to be optimal. These applications of the data are appropriate when the device in question has a color-shape configuration that matches one of the four sign targets used in the laboratory study. For example, if the device of interest were any yellow diamond-shaped warning sign, then the correlation coefficients for the Pedestrian Crossing sign should be used.

\section{a. DETOUR Sign}

Table 7 lists the variables and corresponding correlation coefficients of the factors that were significantly related to recognition of the DETOUR sign. In examining the table, it appears that roadway characteristics that were spatially near to the target were more closely associated with performance than those that were more distant. Evidence of this proximity effect can be inferred from the fact that the majority of the variables related to performance were located in the area adjacent to the sign (i.e. 11 of 17 significant variables describe the surround, and contrast). similarly, the variables with the great- est magnitudes in this table (i.e. $r \geq$ l. 300 i) measure either surround or contrast characteristics.

Table 7. Significant Zero-order Correlations of Complexity variables with Probability of Recognition for DETOUR Sign

$(\mathrm{N}=160, \rho=.155, \mathrm{p}<.05)$

VARIABLES

SCENE - TOTAL

Parked Vehicles: Right

.237

Vehicles: Opposite

$-.194$

Area Type

$-.192$

Il luminance (Photo)

$-.239$

SCENE - LEFT OF CONE

N Units Area: Bright Large Sources

SCENE - IN CONE

N Traffic Signs

SURROUND $-2^{\circ}$

N Bright point Sources

SURROUND $-1^{\circ}$

$N$ Bright Point Sources

$\mathrm{N}$ Bright Medium Sources

Surround Brightness

$-.253$

Surround Average* (Photo) - 250

SURROUND - PERIMETER

N Different surfaces .321 Touching Target

Proportion of Perimeter -.160 Absolute Light

Surround Maximum* (Photo) -.194

\section{CONTRAST}

Proportion of Perimeter

Equal Brightness

Proportion of perimeter

Darker

External Contrast Minimum* .358 (Photo)

\section{b. Pedestrian Crossing Sign}

Table 8 ists the complexity variables that correlated significantly with recognition of the Pedestrian Crossing sign. Here, the dominance of proximal factors noted for the DETOUR sign was not

* $N=120, \rho=.178$ (photometric luminance variables were not measured for targets at 800 feet $[244 \quad n])$. 
present: rather, proximal and distal factors (e.g., scene variables not in the visual cone) were represented equally often. Additionally, perimeter and contrast variables appear less often in Table 8 than in the other three tables. Another trend in this group of variables was that both medium and large sources of bright light - regardless of location within the scene - were associated with the higher rates of recognition; similarly, increases in the amount of other Than Road Dëtail also correlated positively with performance. Another characteristic of the variables related to recognition of the Pedestrian Crossing sign was the failure of any single variable to correlate very strongly with performance; in no instance is the magnitude of the correlation greater than or equal to $1.300 \mathrm{l}$.

Table 8. Significant zero-order Correlations of Complexity variables with Probability of Recognition for Pedestrian Crossing Sign $(\mathrm{N}=160, \rho=.155, p<.05)$

\section{VARIABLES}

SCENE - TOTAL

Other than Road Detail Land Use

Parked Vehicles: Right

Area Type

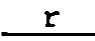

\section{SCENE - LEFT OF CONE}

N Bright Large Sources

N. Units Area: Bright

Large Sources

$\mathrm{N}$ Bright Medium Sources

SCENE - ON ROAD

N Bright Large Sources

SCENE - IN CONE

N Bright Point Sources

SURROOND $-2^{\bullet}$

$N$ Bright Point Sources

$N$ Bright Large Sources

SURROUND $-1^{\bullet}$

N Bright Point Sources

\section{CONTRAST}

Proportion of Perimeter Equal Brightness

Proportion of Perimeter Darker

\section{c. STOP Sign}

Table 9 presents the variables and corresponding correlation coefficients that were significantly associated with subject performance on the STOP sign. One of the most prominent aspects of these data is that more variables correlated with recognition of the STOP sign than with any of the other three targets. of these variables, the majority (14 of 24) were characteristics of the roadway scene that are not immediately adjacent to location of the target. Note also the comparatively large magnitudes of two of these variables (i.e. $r=0.324$ for Other Than Road Detail and $r=-0.318$ for Dry/ Wet Road). At the same time, however, variables describing features near to the sign cannot be discounted by virtue of significant correlations between performance and three perimeter and contrast variables. In fact, considering all of the variables listed in Table 9, performance was most highly associated with Proportion of Perimeter Equal Brightness ( $r=-0.345)$. As was the case with the Pedestrian Crossing sign, both medium and bright sources of light appear to facilitate target recognition. In all, there are eight significant relationships between performance and variables that reflect medium and bright light sources.

\section{d. SPEED ZONE AHEAD Sign}

Table 10 lists the variables that significantly correlated with recognition of the SPEED ZONE AHEAD sign. More than any other device, recognition of this sign appears to have been influenced by variables that measure roadway characteristics adjacent to the target. That is, 14 of the 19 variables correlated with performance reflect features near to the sign (i.e. cone, surround, and contrast variables). Moreover, contrast variables were particularly relevant to recognizing the SPEED ZONE AHEAD sign as shown by both the frequency and magnitude of the correlations. It should be recalled from the previous chapter that the SPEED ZONE sign was the only sign for which brightness and contrast could approach the predictive validity of visual complexity; hence, these contrast variables are useful as single predictors. Finally, as opposed to trends identified for the pedestrian Crossing and STOP signs, medium and bright sources of light did not consistently facilitate target recognition. Only three of these variables were associated with performance, and two of these -- N'Bright Large Sources in the Cone, and $N$ Units Area: Bright Large Sources in the Cone -- were negatively related to recognition. 
Table 9. Significant zero-order Correlations of Complexity variables with Probability of Recognition for STOP Sign

$(\mathrm{N}=160, \quad \rho=.155, p<.05)$

VARIABLES

SCENE - TOTAL

Other Than Road Detail

Dry/Wet Road

Area Type

Road Surface Detail

Luminaires

Road orientation

Land Use

Parked Vehicles: Right

$-.298$

.247

.218

.213

$-.206$

.191

\section{SCENE - LEFT OF CONE}

N Bright Large sources

$N$ Units Area: Bright

Large Sources

$N$ Bright Medium Sources

SCENE - RIGHT OF CONE

$N$ Units Area: Bright Large Sources

N Bright Large Sources

$N$ Bright Medium Sources

SCENE - IN CONE

Cone Brightness

$N$ Traffic Signs

$\mathrm{N}$ Bright Large Sources

.201

$-.192$

$-.171$

SURROUND $-2^{\circ}$

N Bright Point Sources

$N$ Bright Large Sources

SURROUND $-1^{\circ}$

Surround Uniformity

$N$ Traffic Signs

$-.177$

$-.161$

SURROUND - PERIMETER

N Different Surfaces

Touching Target

\section{CONTRAST}

Proportion of Perimeter

Equal Brightness

Proportion of Perimeter Darker
Table 10. Significant zero-order Correlations of Complexity variables with Probability of Recognition for SPEED ZONE AHEAD Sign

$(\mathrm{N}=160, \quad \mathrm{p}=.155, \mathrm{p}<.05)$

\section{VARIABLES}

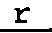

SCENE - TOTAL

Parked Vehicles: Right $\quad .304$

Area Type

$-.284$

Il luminance (Photo)

SCENE - ON ROAD

N Bright Medium Sources

\section{SCENE - IN CONE}

N Bright Large Sources

$-.225$

$N$ Traffic Signs

N Units Area: Bright Large Sources

N Traffic Signs: White

$-.213$

vertical Rectangle

SURROUND $-2^{\circ}$

N Bright Point Sources

Surround Average* (Photo)

SURROUND $-1^{\circ}$

N Bright Point Sources

Surround Brightness

$-.254$

$-.198$

SURROUND - PERIMETER

Proportions of Perimeter

Absolute Dark

Perimeter Brightness

$N$ Different surfaces

Touching Target

\section{CONTRAST}

Proportion of Perimeter Darker

Proportion of Perimeter Equal Brightness

Proportion of Perimeter Lighter

External Contrast Minimum* 
Trends among Variables across Target Type

The simple correlations of individual complexity variables with performance are potentially useful as a means of roughly assessing the relative contribution of complexity variables at a particular roadway location when the target of interest is a traffic sign with a color-shape that is different from those of the four targets used in the laboratory study. By focusing on those variables that correlate significantly with performance across more than one sign some generalizable relationships can be identified. Table 11 ists the variables that were significantly associated with target recognition across three or more signs. In order for a variable to appear in this table, it not only had to be statistically significant for at least three devices, but the direction of the relationship also had to be the same across signs.

A consideration of the implied relationships reveals some commonalities across variables that are consistent with the established principles of target detec tion. On the other hand, some of the relationships observed in the data wer unexpected. The intent of the followi discussion is to offer an interpretatit based on known principles of target detection, of the expected relationshif and draw attention to those associatior that were not expected. This discussic is admittedly speculative and it is als acknowledged that the data are certainl open to alternative explanations. Neve theless, the hypotheses offered represe an attempt to identify some of the processes that underlie the recognition of traffic signs embedded in realistic roadway situations.

An examination of the variables that significantly correlated with performanc across devices suggests that one of the strongest factors that affects the recog nition of a sign target in a roadway scene is the brightness contrast between the target and scene elements that are contiguous to it. In this regard, the

Table 11. Zero-order Correlations of Complexity Factors with Probability of Recognition That Are Significant Across Three or Four Signs

$(\mathrm{N}=160, \rho=.155, \mathrm{p}<.05)$

\begin{tabular}{|c|c|c|c|c|}
\hline \multicolumn{5}{|l|}{ VISUAL } \\
\hline VARIABLES & DTOR & CROS & STOP & SPED \\
\hline SCENE - TOTAL & & & & \\
\hline $\begin{array}{l}\text { Parked Vehicles: Right } \\
\text { Area Type }\end{array}$ & $\begin{array}{r}.237 \\
-.192\end{array}$ & $\begin{array}{r}.257 \\
-.252\end{array}$ & $\begin{array}{r}.191 \\
-.298\end{array}$ & $\begin{array}{r}.304 \\
-.284\end{array}$ \\
\hline SCENE - LEFT OF CONE & & & & \\
\hline N Units Area: Bright Large Sources & .167 & .202 & .248 & \\
\hline SCENE - IN CONE & & & & \\
\hline N Traffic Signs & -.204 & & -.192 & -.225 \\
\hline SURROUND - $1^{\circ}$ & & & & \\
\hline N Bright Point Sources & -.336 & -.262 & & -.254 \\
\hline SURROUND $-2^{\circ}$ & & & & \\
\hline N Bright Point Sources & -.303 & -.353 & -.270 & -.219 \\
\hline SURROUND - PERIMETER & & & & \\
\hline N Different surfaces Touching Target & .321 & & .251 & .169 \\
\hline CONTRAST & & & & \\
\hline $\begin{array}{l}\text { Proportion of Perimeter Darker } \\
\text { Proportion of Perimeter Equal Brightness }\end{array}$ & $\begin{array}{r}.230 \\
-.332\end{array}$ & $\begin{array}{r}.211 \\
-.221\end{array}$ & $\begin{array}{r}.207 \\
-.345\end{array}$ & $\begin{array}{r}.445 \\
-.403\end{array}$ \\
\hline
\end{tabular}


data suggest that recognition is optimal when the target is a bright stimulus within a dark surround. Specifically, as the proportion of Perimeter Darker than the target increased, the probability of recognizing any of the four targets also increased. As the Proportion of Perimeter of Equal Brightness increased, there was a progressive decrement in performance for all four sign targets. These data suggest that brightness contrast is critical to target recognition in heterogeneous environments and that contrast, measured with regard to the percentage of the target perimeter, that is equal or darker, was more closely associated with performance than the photometric measures which integrated the amount of contrast over the entire perimeter.

Just as local brightness contrast affected target recognition, so too did the luminous characteristics of that part of a roadway scene that surrounded a target. The number of bright point sources of light seemed to be particularly influential in this regard. The data indicate that increasing the number of Bright point Sources within a $2^{\circ}$ radius of the target's center significantly interferes with the recognition of all four devices. Likewise, increasing the number of Bright point sources within a $1^{\circ}$ radius also interferes with the recognition of all of the targets except the STOP sign.

While increasing the number of light sources in the area surrounding a target was detrimental to recognition, the dynamics that underlie this phenomenon are largely a matter of speculation. One attractive explanation is that as the observer scans a bright variegated surround, a reduction in the level of contrast sensitivity is induced by transient adaptation. An equally plausible alternative hypothesis is that sources of light adjacent to the sign function as glare sources that operationally cast a veil of light over the target and its immediate surround, thereby decreasing the effective contrast between the target and its surround. A third interpretation is that as the number of point sources in the surround increases, there is a corresponding increase in the amount of discernible detail in the area surrounding the target; the effect of the increased detail could be to camouflage the sign, thereby making it more difficult for an observer to discriminate it as a discrete figure in the middle of a heterogeneous field. Of course, the decrement in performance due to an increased number of light sources in a target's surround could be due to some combination of these three effects.
Another relationship indicated by the lab study data that is consistent with previous research is the deleterious effect of confusing stimuli on performance. That is, as the number of scene elements that resemble the target of interest increase, there is a progressive decrease in probability of recognition. This trend is suggested by significant negative correlations between the Number of Traffic Signs in the cone and performance on all of the targets except the Pedestrian Crossing sign. (Similarly, increasing the Number of white Vertical Rectangles in the cone also interferes with the recognition of the SPEED ZONE AHEAD sign.)

Another variable that was significantly associated with performance across all four sign targets was the presence of Parked Vehicles on the Right Side of the Road. A seemingly legitimate interpretation of this finding is that the parked cars provide a very reliable cue that guides the observer's eye scan through the area of possible target location. other words, parked cars provide road delineation which in turn guides search patterns for traffic signs.

The direction of some of the correlations among the lab data between complexity variables and performance is different from that which was expected on the basis of previous knowledge. Three such variables appear in Table 11. One of the persistent commonalities was the facilitative effect of large bright sources of light on recognition. This effect was noted when the light sources appeared to the left of the cone of possible sign location. Possibly, these light sources acted to aid road delineation and, hence, to improve sign search patterns. Another noticeable trend is that as Area Type becomes progressively more rural, the rate of target recognition decreases. This may simply reflect increased recognition problems at the greater rural distances or the reduction in cues which increases uncertainty or, again, improved road delineation in the more urban settings. The final relationship, improved recognition with an increase in the Number of surfaces Touching the Target, may also be an artifact of distance; near signs having more discriminable surfaces surrounding them.

\section{Regression Analysis}

Multiple linear regression techniques were used to define predictive equations for score complexity. The form of the derived equation is as follows:

$$
y^{\prime}=a+b_{1} x_{1}+b_{2} x_{2}+b_{3} x_{3}+\ldots b_{n} x_{n}
$$


Where $y^{\prime}$ is a predicted sign recognition score, a is a constant, and b's are the weights to apply to the observed value of the corresponding variables ( $\left.X^{\prime} s\right)$.

The predicted $y^{\prime}$ is the estimate or probability of recognition based upon measurement of the variables in the equation. The higher the $R$, the closer $y^{\prime}$ will be to the true or observed probability of recognition. While $R$ increases as the number of predictor variables increases, the $\mathrm{R}$ gradually reaches an asymptotic level so that adding variables beyond a given point may not be worth the trouble of measuring them.

In addition to the decision of how many variables to include, a related problem was the question of whether visual contrast measures should be potential candidates for inclusion in the equation. While not measures of visual complexity, visual contrast might be worth including if it improves the predictive validity and when backgrounds are relatively homogeneous, it may be measured without placement of the actual sign. To evaluate the usefulness of including visual contrast measures in the predictive equations, comparisons were made of R's with and without contrast for both the long or asymptotic equation and equations based upon only the first seven variables. The $R^{\prime} ' s$ are given in Table 12 . The equations (b weights + constant) for each of these regressions is given in Appendix $E$.

The predictive validities of all equations with contrast were higher than those without contrast; however it is questionable whether or not the added validity is worth the difficulty of measuring contrast. The differences observed for Crossing and Detour signs were virtually nil.

To evaluate the effectiveness of these equations to discriminate among good and bad scenes, the equations were used to identify scenes with the best and the worst predicted recognition scores. The five highest complexity (low recognition scores) and five lowest complexity (high recognition scores) scenes were selected at each distance for each target type using the short formula. An analysis of variance was computed for each-target type to determine the main and interactive effects of distance, brightness, and complexity. The level of significance for all $F$ ratios computed in these analyses is reported in Table 13 .

In general, all $F$ ratios for main effects were significant while those for interactions were inconsistent. It is significant to note that the $F$ ratio for the main effect of complexity was only significant at the .05 level for the pedestrian crossing sign, but at the .001 level for the other signs. This is consistent with the finding that performance was significantly better with the Pedestrian Crossing sign. While the dimensions of signs were confounded within the four signs studied, it seems reasonable to hypothesize that the shape of the Pedestrian Crossing sign was instrumental in its achieving the highest recognition rate and that this shape also served to offset the detrimental effects of highway complexity.

The interactions of brightness by complexity are shown in Figure 6. Although only one of the $F$ ratios for this interaction was significant, the figure is included because it graphically reveals confirmation of our central hypothesis about sign conspicuity. Conspicuity is change in the sign or its surround necessary to offset decrements in visual performance resulting from scene complexity. The line representing the low brightness level signs in Figure 6 shows the drop in performance due to complexity. The graphs for all but the SPEED ZONE sign reveal that performance with high brightness - high complexity is back to where it was with the low brightness sign at low complexity. For the SPEED ZONE sign, brightness fails to make up the performance decrement occurring from complexity. This may well be because the brighter this sign becomes the more it looks like a point source and recognition as a traffic sign does not occur.

Table 12. Multiple R's Based upon Long (L) and Short (S) Equations with and without Contrast for Four Target Types

\begin{tabular}{|c|c|c|c|c|c|c|c|c|}
\hline & \multicolumn{2}{|c|}{ STOP } & \multicolumn{2}{|c|}{ DTOR } & \multicolumn{2}{|c|}{ SPED } & \multicolumn{2}{|c|}{ CROS } \\
\hline & L & $s$ & $\mathrm{~L}$ & $\mathrm{~s}$ & $\mathrm{~L}$ & $\mathrm{~s}$ & $\mathrm{~L}$ & $s$ \\
\hline without Contrast & .65 & .55 & .79 & .47 & .75 & .62 & .64 & .55 \\
\hline with contrast & .74 & .66 & .80 & .49 & .80 & .72 & .66 & .58 \\
\hline
\end{tabular}



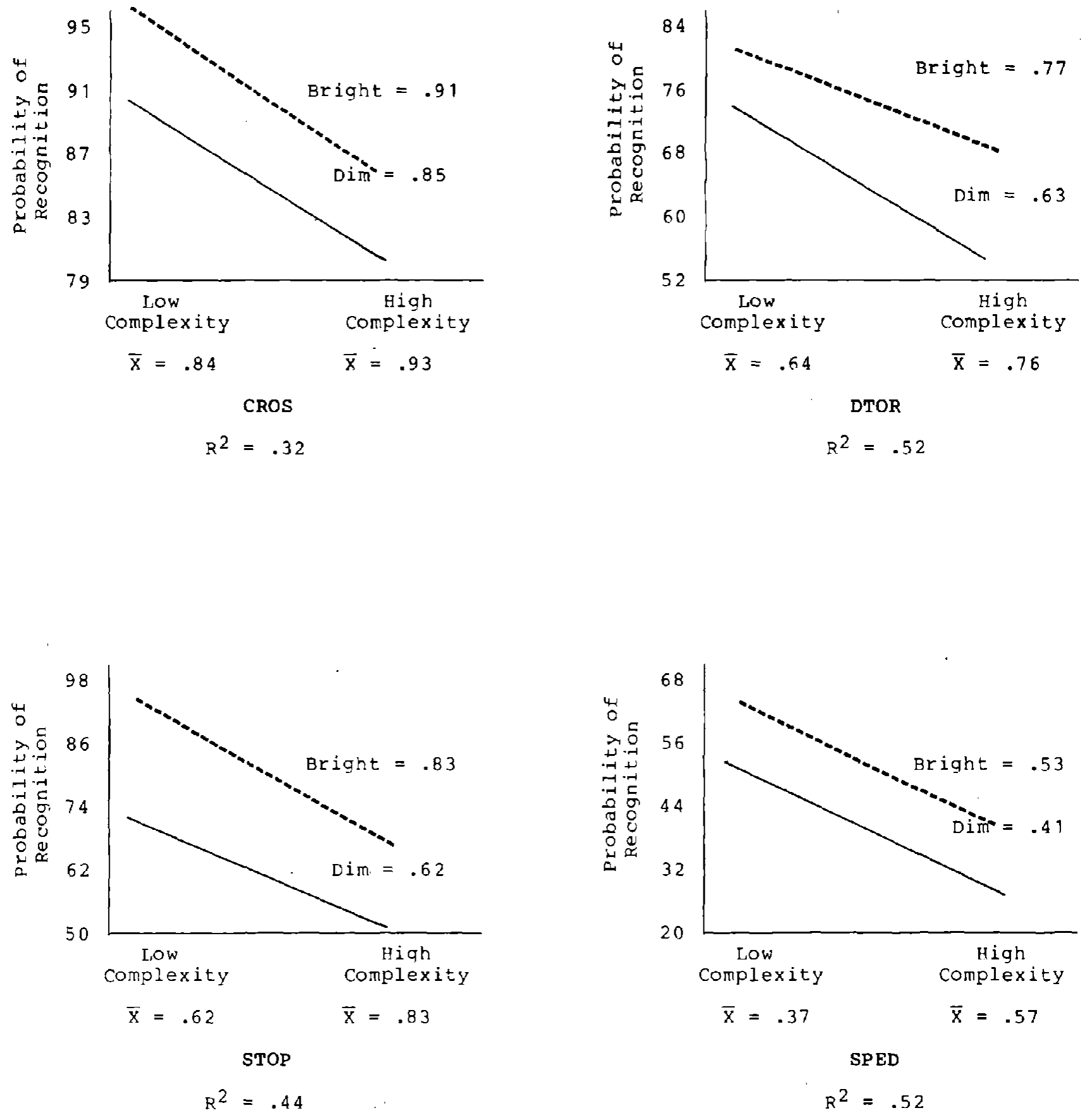

Figure 6. Interaction of Visual complexity and Brightness for Four Traffic signs. 
Table 13. F-Ratios for Main and Interactive Effects of Distance, Complexity, and Brightness for Each Experimental Sign

\begin{tabular}{|c|c|c|c|c|c|}
\hline Short Formula & $\underline{\mathrm{DF}}$ & STOP & DTOR & CROS & SPED \\
\hline Distance (A) & 3 & $21.14 \star \star \star \star$ & $51.84 * \star \star$ & $21.03 \star \star \star$ & $23.67 \star \star \star$ \\
\hline Complexity (B) & 1 & $13 \cdot 70 \star \star \star$ & $12.39 * \star \star$ & $7.06 *$ & $26.76 \star \star \star$ \\
\hline Brightness (C) & 1 & $126.49 * \star \star$ & $38.00 * * \star$ & $25.81 \star \star \star$ & 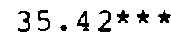 \\
\hline$A \times B$ & 3 & 2.29 & $5.13^{\star \star}$ & $3.78^{\star}$ & $4.95 * \star$ \\
\hline$A \times C$ & 3 & $10.45 * * *$ & 2.58 & $3.27^{\star}$ & 1.27 \\
\hline $\mathrm{B} \times \mathrm{C}$ & 1 & .05 & $4.18 *$ & .01 & .64 \\
\hline$A \times B \times C$ & 3 & 1.52 & .66 & 2.76 & $2: 44$ \\
\hline
\end{tabular}

\section{E. DISCUSSION}

The laboratory results build a strong case for the usefulness of visual complexity as a predictor of detection and recognition. The results indicate that at locations within complex visual fields, visual measures of the scene and the sign's surround predict visual performance better than either visual or photometric measures of sign contrast and brightness. Also encouraging was the finding that increasing sign brightness can offset the degrading effects of higher visual complexity.

The measures of visual complexity, sign brightness and contrast should provide a useful contribution to future research. Methods for measuring contrast, surround, and other variables, had not previously been defined for complex stimuli. The photometric methods for measuring internal contrast should also be of benefit. The fact that visual measures can have significant validity and be reliably coded suggests the potential for a practical method whereby field personnel can judge the.visual complexity of a location and its effect on driver recognition of traffic signs.

As mentioned elsewhere, the absence of specific a priori hypotheses precluded a theoretical approach and made the reliability of the regression equations essentially indeterminate. Nevertheless, the consistency and pattern of results suggests that the measures are reasonably reliable. Additional work is now needed to refine the visual measures which we

have presented and to formulate and test hypotheses about their interrelationships.

\section{FIELD STUDY}

\section{A. PURPOSE}

Both the laboratory and field research efforts of this project were designed to. assess traffic sign recognition as a function of both device luminance and visual complexity. The laboratory study examined the recognition of four traffic control devices that varied in shape, color, and legend. The result of this study indicated that sign recognition is degraded by visual complexity and that increasing sign luminance offsets this decrement in performance. The intent of the field study was to determine whether: these relationships also exist in the real world. The field study represented an attempt to measure the effect of luminance on sign recognition in visually different roadway settings under realworld conditions. Two specific hypotheses were tested by the field study. The first of these was that the probability of a driver recognizing a traffic sign increases as the luminance of the device increases. The second hypothesis tested was that the probability of a driver recognizing a traffic sign increases as the visual complexity of the roadway environment decreases. Two similar hypotheses assessed by the study stated that the distance at which a driver recognizes a traffic sign increases as sign luminance increases and as visual complexity decreases.

$$
\begin{aligned}
\star \star \star p & <.001 \\
\star \star p & <.01 \\
\star p & <.05
\end{aligned}
$$




\section{B. EXPERIMENTAL METHOD}

\section{Methodológy}

The selection of a methodology to test these hypotheses was guided by consideration of the role of conspicuity in nighttime sign detection by real-world drivers. In order to draw conclusions about luminance effects on sign recognition that are valid in the real world, an attempt was made to collect the field data under conditions that were as naturalistic as possible. Toward this end, a free-field observational procedure was emloyed as the methodological approach of choice. The primary thrust of this approach was to use inconspicuous techniques to measure traffic performance in response to a controlled treatment condition. Specifically, the field data collection procedure involved the unobtrusive measurement of changes in the speed of subject vehicles in response to a diamond-shaped, yellow warning sign with the legend SPEED TRAP. To the extent that this procedure minimized experiment-induced sensitivity to the target sign among the subject drivers, the field data should be representative of the behavior of real-world drivers under natural conditions.

Although this approach may have maximized the external validity of the field study, overt vehicle behavior (i.e., vehicle speed profiles) had to be used as a surrogate measure of sign recognition. In general, vehicle behavior can be used as än index of recognition only if the recognition of a particular sign consistently stimulates an uninterrupted sequence of sign detection, recognition, decision, and reaction. The SPEED TRAP sign was chosen for use in the field study on the assumption that speeding drivers are sufficiently motivated to detect and recognize such messages and will then promptly decrease vehicle speed.

This assumption appears to have face validity. That is, the experience of paying a fine for speeding would seem to be sufficiently aversive that drivers generally would be motivated to avoid that situation when given a cue that apprehension by enforcement authorities is likely. In spite of this face validity, it was considered necessary to actually demonstrate that speeding drivers do, in fact, slow down as soon as possible after recognizing the SPEED TRAP sign. For this reason, a pilot study was conducted at two highway sites to determine whether the sign elicits an observ- able response. The results of the pilot provided three observations. First, speeding drivers did decelerate when the SPEED TRAP sign was deployed. Second, the frequency of decelerations was greater when an array of tape switches (used to record vehicle speeds) were deployed together with an unmarked, shoulder-parked passenger car; and, third, vehicles did not respond to the tape switches and car when the sign was not present. These findings were interpreted as indicating that while the sign has an impact, the presence of the car lends credibility to the message of the sign, thereby sufficiently motivating drivers to respond to the device. On the basis of this pilot study, it was concluded that the SPEED TRAP sign could justifiably be used in a methodology that purports to measure vehicle speed profiles as an indication of target sign recognition. However, in a methodology concerned with location of sign recognition, recognition of the car must precede recognition of the sign's message to ensure that response initiation is elicited by the sign as opposed to the car.

\section{Site Selection}

The major objective of the field study was to explore the effect of different levels of sign luminance across a range of levels of visual complexity. As a result, controlled variation in visual complexity was a desired feature of the experimental methodology. However, the only practical method available to vary complexity was by site selection. An attempt was made to select three different highway sites that were as closely matched as possible in terms of both roadway geometrics and the operational traffic situation but systematically different in terms of level of complexity. The effort to match the sites, however, was limited to some extent by requirements imposed by both the instrumentation used for data collection and the experimental methodology itself.

The Traffic Evaluator System (TES) was required to record vehicle speeds at each site. The TES is a hard-wire system that records momentary closures in electronic circuits that are actuated by wheel hits on a series of tape switches deployed on the surface of the road throughout the site.* An integral component. of this system are several main data cables that transmit impulses from all of the tape switches to an on-site electronics unit. These linkage cables run longitudinally along the road throughout the site, and

\footnotetext{
* A complete description of the system is available in the Traffic Evaluator System Manual. Seguin, et al. (1982).
} 
their physical characteristics are such that they cannot be run over by any kind of vehicle. Because of this, the sites selected for data collection had to have either a shoulder or a median that was uninterrupted by driveways. This requirement proved to be a significant restriction to the universe of deployable sites, especially since visual complexity tends to be positively related to the number of access points to a highway. This restriction on sites, in turn, necessitated compromises in the degree to which the sites could be matched.

Similar restrictions on the number of sites amenable to data collection were imposed by roadway cross-section and longitudinal requirements dictated by the experimental methodology. With regard to cross-section features, the desirability of generalizing the findings to the most typical problem situations mandated that the sites be located on a four-lane highway, with two lanes of traffic moving in each direction of travel. The second cross-section restriction concerned the shoulder of the road. The need to position the unmarked passenger car on the shoulder of the road just upstream of the target sign made it essential that the roadway have a shoulder at least 10 feet ( $3.05 \mathrm{~m})$ wide to ensure that the parked car did not generate changes in either the lateral placement or speed profiles of stream vehicles.

The experimental methodology also dictated specifications pertinent to the longitudinal characteristics of the roadway on either side of the target sign. First, the desire to observe a large number of speeding vehicles led to the requirement that the target sign be at least $1500 \mathrm{ft}$. $(457.5 \mathrm{~m})$ beyond the nearest signalized intersection and at least $2000 \mathrm{ft}$. $(610 \mathrm{~m})$ prior to the next signalized intersection. A related requirement specified that traffic signals downstream from the site must not be visible to drivers passing through the site. Second, it was considered necessary that the initial sign visibility distance either be matched across sites or else be greater than 1000 feet $(305 \mathrm{~m}$ ) across sites. The rationale for this requirement was that the duration of time that a traffic sign is available for detection by a driver is likely to affect detection rates significantly and thus confound the influence of sign luminance and/or level of complexity. Moreover, it was felt that beyond 1000 feet $(305 \mathrm{~m})$, additional increases in initial sign visibility distance are negligible since it is very unlikely that a driver's preview for traffic signs is greater than 1000 feet $(305 \mathrm{~m})$ - or 12.3 seconds at a speed of $55 \mathrm{mph}(88.495 \mathrm{~km} / \mathrm{hr})$. Einally, it was considered desirable that there be no changes in either the horizontal or vertical alignment of the road for the 1000 -foot $(305 \mathrm{~m})$ approach to. the target sign. This requirement was necessary because one of the independent variables of the study was sign Iuminance. In a study of sign luminance, roadway approach geometrics are relevant because the luminance of a retroreflective device varies as a function of its position within the iso-candela beam pattern of the headlamps of approaching vehicles. Consequently, from any given approach distance, level of sign luminance is equivalent across sites only if the approach to the sign is without horizontal and vertical curves.

The limitations imposed by the experimental methodology and the requirements of TES deployment reduced the number of candidate sites so that visual complexity played a lesser role in the selection of sites. The overall strategy employed in the site selection process was to weigh individual site characteristics in terms of apparent relevance to the hypotheses being tested and then to select, from those sites that were TES deployable, the three sites that were best-matched on the relevant independent variables. All of the variables considered in this process, together with the values associated with each data collection site, are listed in Table 14. An examination of this table suggests that for those variables that could not be perfectly matched, the discrepancies across sites do not appear to be particularly salient. It should be noted, however, that since the level of visual complexity is a between-site variable, the potential effect of differences in site characteristics might have confounded the influence of complexity level.

\section{Apparatus}

The apparatus used in the field study consisted of the SPEED TRAP sign, the TES used to record vehicle trajectory data, and the shoulder-parked passenger car which was deployed to lend credibility to the message of the target sign. The target sign was designed to resemble a standard warning sign in order to maximize generalizability of the influence of this class of device. The target appeared as a 36 inch $(91.44 \mathrm{~cm})$ yellowdiamond with a black legend. The 36 inch ( $91.44 \mathrm{~cm})$ size was chosen because Standard Highway Signs (FHWA, 1979) indicates that this is the "standard" size for similar literal warning signs (e.g. STOP AHEAD - w3-1). An illustration of the target sign and its dimensions is provided in Figure 7. The sign was displayed on a portable sign mount that was designed to be sturdy, inconspicuous, and 
Table 14. Characteristics of Sites selected for Field Data Collection

\begin{tabular}{|c|c|c|c|}
\hline \multirow[b]{2}{*}{$\begin{array}{c}\text { ROADWAY } \\
\text { CHARACTERISTICS }\end{array}$} & \multicolumn{3}{|c|}{ DATA COLLECTION SITES } \\
\hline & $\begin{array}{l}\text { Dillworthtown } \\
\text { US } 202 / 322 \mathrm{~S} \\
\quad \pm \pm \\
\text { low complexity }\end{array}$ & $\begin{array}{c}\text { Pittsburgh } \\
\text { PA } 65 \mathrm{~S} \\
\star \pm \star \\
\text { medium complexity }\end{array}$ & $\begin{array}{c}\text { Greensburg } \\
\text { US } 30 W \\
\star \star \star \\
\text { high complexity }\end{array}$ \\
\hline $\begin{array}{l}\text { CROSS SECTION } \\
\text { CHARACTERISTICS }\end{array}$ & & & \\
\hline $\begin{array}{l}\text { number lanes } \\
\text { each direction }\end{array}$ & 2 & 2 & 2 \\
\hline lane width & $10^{\prime} 9^{n *}$ & $9^{\prime} 10^{\prime \prime}$ & $11^{\prime}$ \\
\hline pavement type & blacktop & blacktop & concrete \\
\hline $\begin{array}{l}\text { pavement condition } \\
\text { shoulder width }\end{array}$ & $\begin{array}{c}\text { few cracks, no holes } \\
8^{\prime} 9^{\prime \prime} \text { to } 13^{\prime} 3^{\prime \prime}\end{array}$ & $\left\{\begin{array}{c}\text { few cracks, no holes } \\
0 " \text { to } 14^{\prime} 3^{\prime \prime}\end{array}\right.$ & $\begin{array}{c}\text { few cracks, no holes } \\
13^{\prime} 8^{\prime \prime}\end{array}$ \\
\hline median type & $\begin{array}{l}\text { aluminum guardrail } \\
\text { on a concrete base }\end{array}$ & double yellow line & $\begin{array}{l}\text { aluminum guardrail } \\
\text { on gravel and/or } \\
\text { concrete base }\end{array}$ \\
\hline median height & $2^{\prime} 6 "$ & 0 & $2^{\prime}$ \\
\hline $\begin{array}{c}\text { median width } \\
\text { LONGITUDINAL } \\
\text { CHARACTERISTICS }\end{array}$ & $3^{\prime}$ & $I^{\prime} 3 "$ & $8^{\prime}$ to $19^{\prime}$ \\
\hline $\begin{array}{c}\text { initial sign } \\
\text { visibility distance }\end{array}$ & $2010^{\prime}$ & $1100^{\prime}$ & $1450^{\circ}$ \\
\hline $\begin{array}{c}\text { distance from prior } \\
\text { traffic signal to } \\
\text { target sign }\end{array}$ & $2100^{\prime}$ & $2100^{\prime}$ & $2430^{\prime}$ \\
\hline $\begin{array}{l}\text { distance from } \\
\text { target sign to next } \\
\text { traffic signal }\end{array}$ & $4450^{\prime}$ & $>1 \mathrm{mile}$ & $>1 \mathrm{mile}$ \\
\hline $\begin{array}{l}\text { horizontal } \\
\text { alignment: } \\
1000^{\prime} \text { approach to } \\
\text { sign }\end{array}$ & tangent & $\begin{array}{l}\text { point of tangency } \\
\text { of flat curve } \\
\text { right } 650^{\prime} \\
\text { upstream of target }\end{array}$ & tangent \\
\hline $\begin{array}{c}\text { horizontal } \\
\text { alignment: } \\
1000^{\prime} \text { approach to } \\
\text { sign }\end{array}$ & tangent & tangent & $\begin{array}{l}\text { point of curvature } \\
\text { of flat curve left } \\
500 \text { ' downstream of } \\
\text { target }\end{array}$ \\
\hline $\begin{array}{c}\text { vertical alignment: } \\
1000^{\prime} \text { downstream } \\
\text { of sign }\end{array}$ & level & level & $\begin{array}{c}\text { constant slight } \\
\text { upgrade }\end{array}$ \\
\hline $\begin{array}{c}\text { vertical alignment: } \\
1000^{\prime} \text { downstream of } \\
\text { sign }\end{array}$ & $\begin{array}{c}\text { PC of slight upgrade } \\
\text { at } 700 \text { ' downstream } \\
\text { of target }\end{array}$ & $\begin{array}{c}\text { PC of slight } \\
\text { downgrade at } 700^{\prime} \\
\text { downstream of target }\end{array}$ & $\left\{\begin{array}{c}\text { start of level } \\
\text { section at } 200^{\prime} \\
\text { downstream of target }\end{array}\right.$ \\
\hline
\end{tabular}

* One foot $=.305^{\circ}$ meters.

One inch $=2.540$ centimeters.

one mile $=1.690 \mathrm{kilometers.}$ 
Table 14. Characteristics of Sites Selected for Field Data Collection (continued)

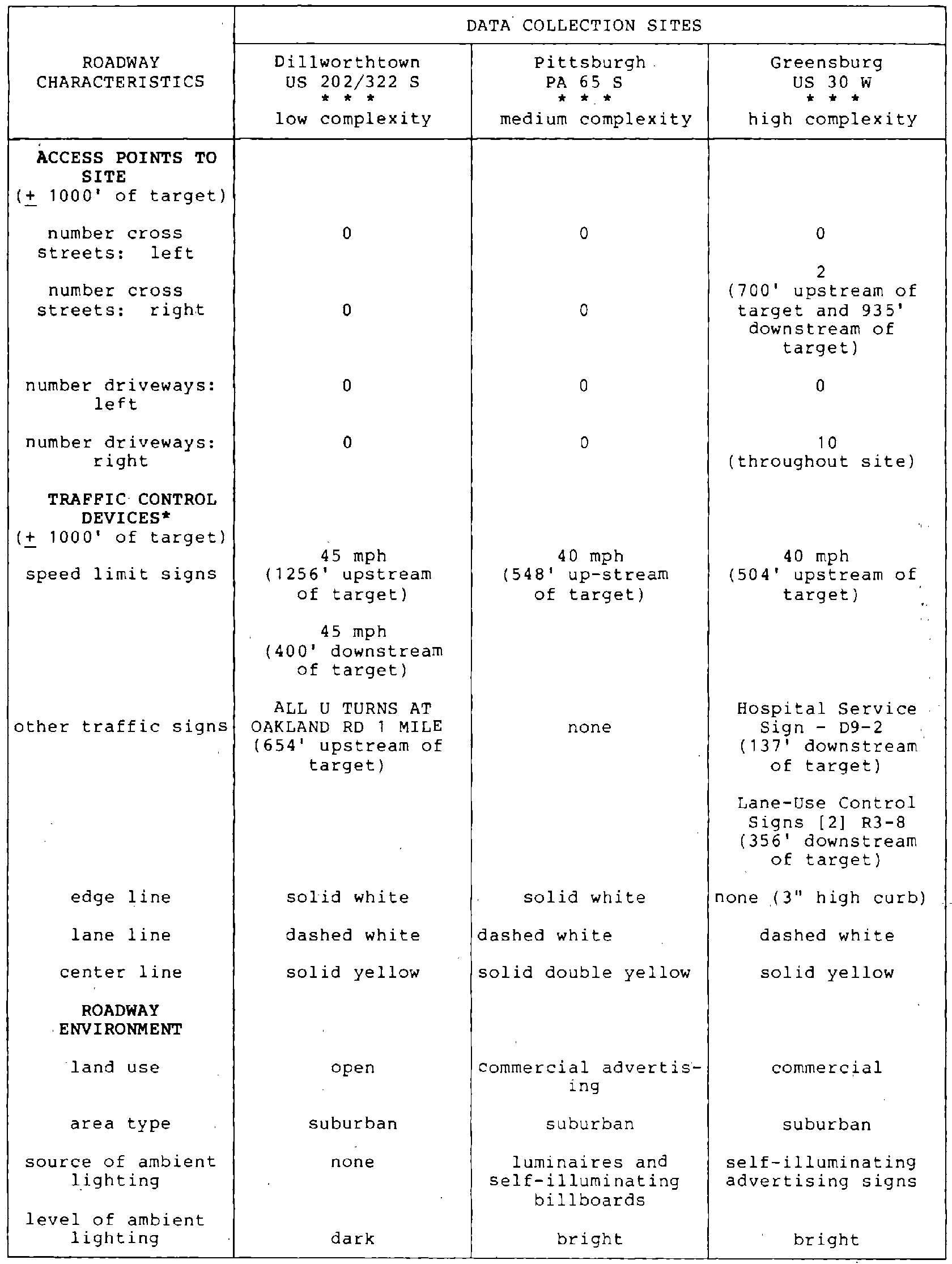

* All of the traffic signs at each site were shoulder-mounted devices. 
Table 14. Characteristics of sites Selected for Field Data Collection (continued)

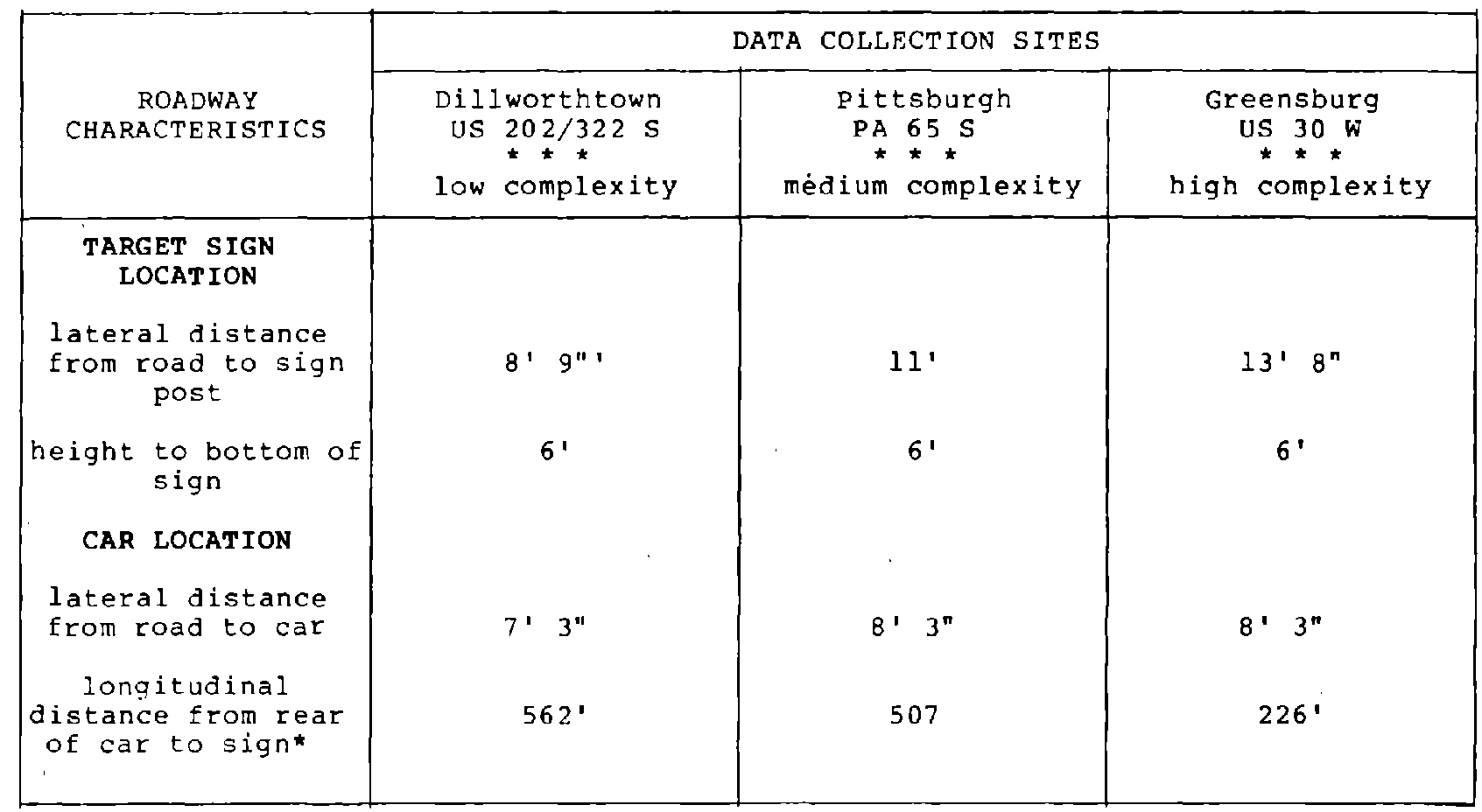

similar to the typical standards for shoulder-mounted signs. This mount was constructed of dark green channel steel and consisted of a single vertical pole anchored by a heavy base. The base was made of a stout cylinder of dark grey concrete and three horizontal, two-foot $(.610 \mathrm{~m})$ legs that were also dark green channel steel.

At each site, the target sign was positioned midway in a 1200 foot (366 m) course that was instrumented via the TES. The TES was used to develop a speed profile for each vehicle that traversed the measured course. This profile consisted of a total of eight speed measurements, each of which represented the vehicle's average velocity over the 150 foot $(45.75 \mathrm{~m})$ interval between adjacent TES traps. The first of these traps was located 600 feet $(183 \mathrm{~m})$ upstream of the target sign, and the ninth trap was 600 feet $(183 \mathrm{~m})$ downstream of the target sign. A trap consisted of a parallel pair of tapeswitches affixed to the road surface perpendicular to traffic flow and spaced precisely four feet apart. Each tapeswitch was attached to the pavement surface via olive drab duct tape, which helped to make the switches visually obscure. Since each switch measured only $3 / 16$ inches $(.476 \mathrm{~cm})$ high, switches did not appear to create sufficient audible or tactile feedback to drivers to influence speeds. The TES deployment at each site consisted of nine traps in each of the two lanes of travel, so that a total of 36 tapeswitches were installed per site. These switches were linked via main data cables to an on-site electronics unit that recorded the data on magnetic computer tape. The power source for the system was a standard 12 volt automotive battery. At each site, the cables, electronics unit, and battery were hidden from the drivers' field of view.

The basic datum that is recorded with this system is the arrival time of an axle over a particular switch. The input from a single trap permits a fairly accurate determination of the number of axles and the wheelbase of each vehicle in the system as well as a record of the spot speed of each vehicle over the trap. Given the number of axles, the wheelbase, and vehicle speed, and allowing for inter-trap acceleration and deceleration possibilities, projections downstream to successive traps can be used to define a "window" of time within which the vehicle's axles must arrive in order to be identified as belonging to the same vehicle. This provides a reasonably precise record of the amount of time that

* The nature of the shoulder of the road at each site dictated the longitudinal position of the shoulder-parked car. 


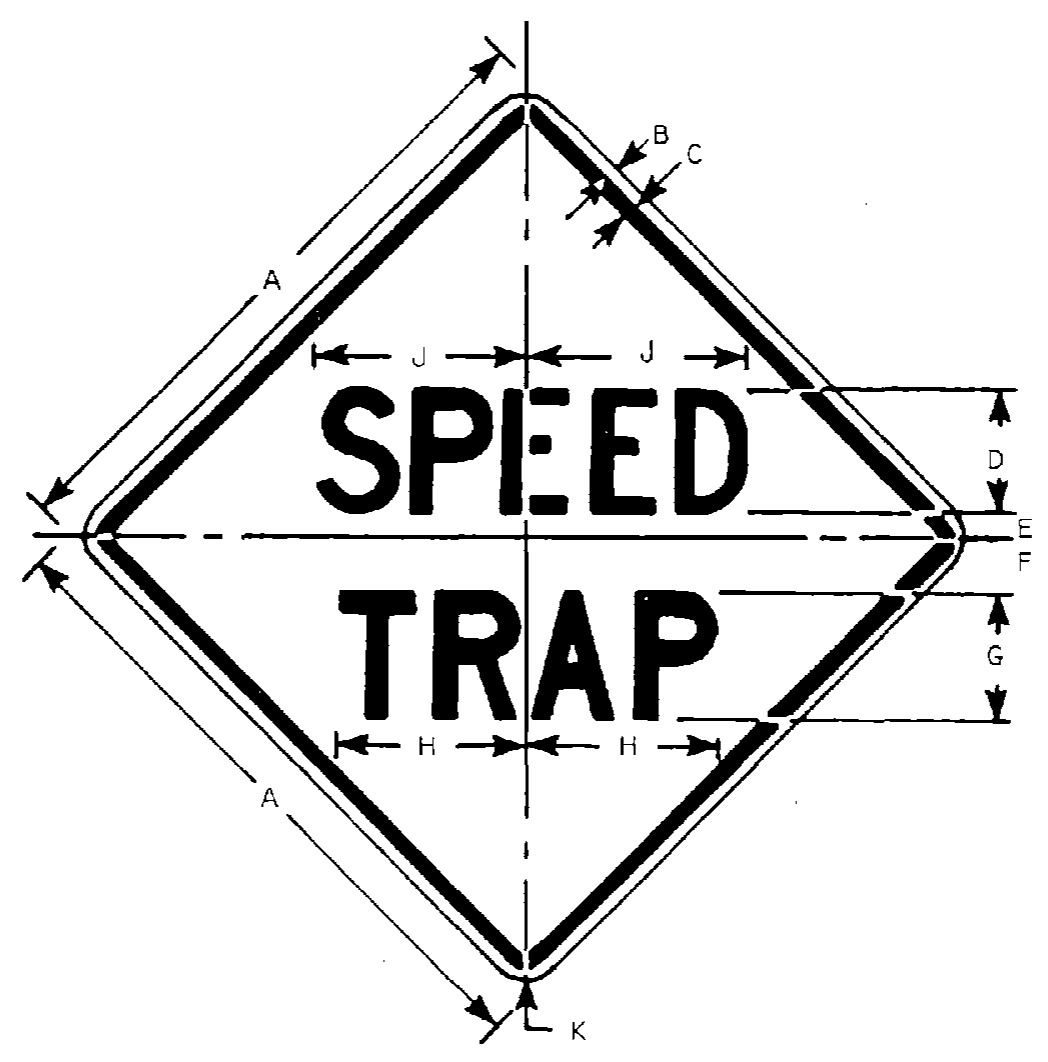

\begin{tabular}{|c|c|c|c|c|c|c|c|c|c|}
\hline \multicolumn{10}{|c|}{ DIMENSIONS (INCHES)* } \\
\hline A & B & $\mathrm{C}$ & D & $E$ & F & G & $\mathrm{H}$ & $\mathrm{J}$ & $\mathrm{K}$ \\
\hline 36 & $5 / 8$ & $7 / 8$ & $7 \mathrm{C}$ & $11 / 2$ & 3 & $7 \mathrm{D}$ & 11 & $121 / 4$ & $21 / 4$ \\
\hline
\end{tabular}

COLORS

$$
\begin{array}{ll}
\text { LEGEND } & - \text { BLACK (NON-REFL) } \\
\text { BACKGROUND } & - \text { YELLOW (REFL) }
\end{array}
$$

Figure 7. Target Sign and Dimensions

* One foot $=.305$ meters.

One inch $=2.540$ centimeters. 
transpires as a vehicle traverses each 150 foot $(45.75 \mathrm{~m})$ interval between adjacent traps. With this output, existing computer programs were used to generate vehicle speed profiles over the measured course and to identify vehicle types on the basis of number of axles and length of the wheelbase. In addition, headways, and mean speeds over each trap were also calculated. While the error of a single trap input is limited to $.06 \mathrm{msec}$, a large number of factors determine the accuracy of speeds and headways estimated by the software. In general, the overall accuracy is within 4 percent for all computed measures.

The only other equipment used in the field study was the unmanned passenger car parked on the shoulder of the road. The purpose of the car was to lend credibility to the SPEED TRAP sign and thereby increase the probability that the sign would have an observable effect on speeding vehicles. At the same time, however, it was important for speeding vehicles not to decelerate in response to the car without the sign. In order for the car to function effectively in both situations, it was important for the car to look as if it could be a police vehicle when seen in conjunction with the sign and to appear as an ordinary passenger vehicle when the sign was not present. In addition, it was considered desirable that the vehicle's rear reflective markings facilitate its detection and subsequent recognition as a vehicle. The car used for the field study was judged to satisfactorily meet these three criteria. This car was a 1980 Chevrolet Malibu Classic; it was a navy-blue four door sedan. Figure 8 illustrates how the car appeared at the Pittsburgh (medium complexity) site to drivers in the shoulder lane 100 feet $(30.5 \mathrm{~m})$ upstream of the rear of the vehicle.

\section{Data Collection Procedure}

Data were collected on consecutive weekday nights at each of the three sites. The first night of data collection at each site was used to gather control data, which were used to identify the typical speed pattern through each site, uninfluenced by the SPEED TRAP sign. The only difference between the control and treatment conditions was the absence of the target sign on the control nights. The time of night during which data were collected varied minimally from one night to the next; these times ranged from 6:00 P.M. at the earliest to $2: 30 \mathrm{~A} . \mathrm{M}$. at the latest. Minor variations in start-up times were attributable to system hardware problems that required remediation prior to the onset of data collection on a given night. Data collection was limi- ted, however, to hours of full dark; that is, data were never gathered during the twilight conditions of dusk. Finally, each night's data were recorded under clear, dry conditions. Data were not recorded if the atmosphere was foggy, if the pavement was wet, or if there was condensation on the target sign.

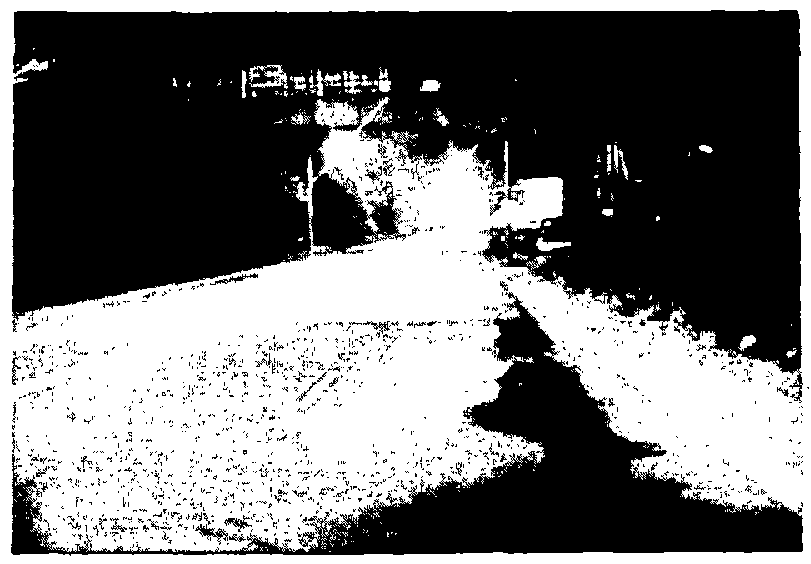

Figure 8. Photograph of the Shoulderparked Car at the Pittsburgh (Medium Complexity) site,

Taken 100 Feet $(30.5 \mathrm{~m})$ Upstream of the Rear of the vehicle

Data collection was effected by a crew of four men experienced in the deployment and operation of the TES. The data collection tasks included site preparation, operation and maintenance of the TES, and system tear down after the completion of data collection at each site.

All site preparation tasks were completed prior to data collection. The TES was deployed during the night preceding data collection between the hours of midnight and 6:00 A.M. System deployment involved affixing the tapeswitches to the surface of the road, making the necessary electrical connections, and camouflaging the electronics unit and main data cables. The only other site preparation required the field crew to position the shoulderparked car and the target sign in the appropriate locations immediately before the onset of data collection each night. of course, on control nights the target sign was not deployed.

During data collection the field crew monitored the operation of the TES, provided manually coded inputs to the TES, and changed the target sign. Operation of the TES required the system to be started and then checked at regular intervals to ensure that data were being recorded on the magnetic computer tape and that the tapeswitches were opera- 
tional. Damaged switches were changed on a nightly basis both before and after the actual hours of data collection.

The coding task was handled by the four crew members on a rotating basis. At any given time during data collection, two observers each monitored half of the measured course - either the 600 feet $(183 \mathrm{~m})$ just upstream of the target sign or the 600 feet $(183 \mathrm{~m})$ just downstream of the sign. In general, the observers coded any event that potentially interfered with the speed of mainstream vehicles. Some of these events involved activity on the shoulder of the road. Examples of such events included: stationary or moving vehicles poised to enter the mainstream and pedestrians walking near the edge of the road. other events that were coded pertained to activity on the road itself. Such events included animals on the road and marked police vehicles passing through the site. The beginning and end of each event was coded as an input to the TES magnetic tape, and this information was subsequently used to delete subject vehicles whose speed profiles may have been influenced by the events coded. In deleting vehicles, conservative estimations were made so as to exclude those mainstream vehicles that may have previewed a coded event as well as those vehicles that may have been affected by the acceleration of merging vehicles.

The final task that was executed during data collection was changing the target sign at specified times throughout each non-control night of data collection. These sign changes were the means by which sign luminance was varied. Data recorded just before, during, and after these changes were subsequentiy deleted.

The sign, and its mount -- as well as the shoulder-parked car -- were removed from the site at the end of each night of data collection. All additional apparatus were removed from the site immediately following the final night of data collection.

\section{Subjects}

The subject sample for the field study was restricted to motorists exceeding the posted speed limit, because only these drivers could be expected to have been sufficiently motivated to detect, recognize, and respond to the message of the SPEED TRAP sign. In the state of Pennsylvania, it is a fairly common belief among motorists that speeding citations are almost certainly given to those drivers that are in violation of the posted limit by $6 \mathrm{mph}(9.654 \mathrm{~km})$ or more. Because of the widespread nature of this belief, the sample was restricted to vehicles whose measured speed over the first 150 feet $(45.75 \mathrm{~m})$ of the course was more than $6 \mathrm{mph}$ above the limit.

Since decreases in speed that were due to influences other than the SPEED TRAP sign constituted a source of error variance, the subject sample was further limited to vehicles which appeared to be uninfluenced by other vehicles on the road. Specifically, each subject vehicle was required to maintain at least an 8 second clear headway throughout the 1200 foot $(366 \mathrm{~m})$ measured course. A vehicle travelling $55 \mathrm{mph}(88.49 \mathrm{~km} / \mathrm{hr})$, for example, had to have a headway distance of at least 645 feet $(196.725 \mathrm{~m})$. Another extraneous source of speed reductions among stream vehicles may have been activity on either the shoulder of the road or on the road itself. Examples of such activity included vehicles poised to merge into the mainstream, pedestrians walking near the edge of the road, and marked police cars passing through the site. These kinds of events were coded manually by members of the field crew during the hours of data collection, and subject vehicles that may have been in- $:$ fluenced by such activities were subsequently deleted from the sample.

Because of the greater observation angles and slower performance characteristics associated with larger trucks, the subject sample for the field study was limited to passenger cars, vans, and pick-ups - with no distinctions made among these subgroups. Further, to eliminate those vehicles whose speed related behaviors may have been influenced by lane change maneuvers, only those vehicles that did not change lanes within the study site were included.

\section{Independent Variables}

The primary objective of the field study was to determine how sign recognition is affected by both level of device luminance and level of visual complexity. Toward this end, both sign luminance and visual complexity were systematically varied as independent variables. Three levels of sign luminance were presented at each of three data collection sites that differed in terms of complexity.

Sign luminance was varied by using three target signs that differed only with regard to the type of sheeting with which each sign was constructed. The high luminance condition employed a sign made from new Type III sheeting. The medium luminance sign was made from new Type II sheeting. The low luminance sign featured new Type II sheeting that was artifically degraded to simulate the appear- 
ance of a weathered Type II sign. This sign was degraded by stretching standard hardware cloth (16 squares per inch) across the face of the sign. This screen-like cloth was kept taut by a web of elasticized cords stretched across the back of the sign. The hardware cloth was made of galvanized steel that was thoroughly covered with flat-black acrylic spray paint. A nighttime photograph illustrating the target sign partially covered with hardware cloth is presented in Fiqure 9.

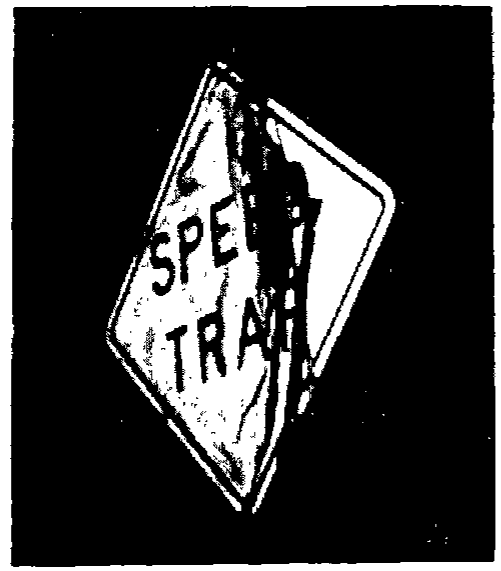

Figure 9. Nighttime Photograph of the Target sign

partially Covered with Hardware Cloth

Because sign luminance was varied by using sheeting with different levels of specific reflectance, and because legibility as well as detection varies as a function of specific luminance, it was considered necessary to estimate the legibility distances associated with each of the luminance conditions. Table 15 illustrates the relevant relationships. For reference, the minimum specific luminance requirements specified for different types of new yellow sheeting are listed in the first column of this table (Standard Specifications for Construction of Roads and Bridges on Federal Highway Projects, FHWA, 1979). The second column of the table presents the average specific luminance of each of the target signs used in the field study. This average is the mean of five independent measures of the sign background taken with a $910-\mathrm{B}$ Gamma Scientific Retroreflectometer, using an entrance angle of $-4^{\circ}$ and an observation angle of $0.2^{\circ}$. A comparison of the first two columns in this table indicates that the low, medium, and high luminance devices correspond very closely to the minimum specifications of Types I, II, and III sheeting, respectively. The average actual specific luminance data were used as input for predicting the 85 th percentile legibility distances associated with each of the three signs. These legibility distances, listed in the third column of Table 15, were calculated by using olson and Bernstein's (1977) predictive legibility curve for a roadside sign illuminated by low beams and situated 12 feet $(3.66 \mathrm{~m})$ to the right of the pavement surface. Given that the letter height of the legend on the SPEED TRAP sign is 7 inches $(17.78 \mathrm{~cm})$, the 85 th percentile nighttime legibility distances predicted for the low, medium, and high brightness signs are 315,329 , and 336 feet $(96.075,100.345$, and $102.48 \mathrm{~m})$, respectively. This suggests that the legibility distance associated with the high luminance sign is only 21 feet $(6.405 \mathrm{~m})$ or 7 percent greater than that for the low luminance device. Moreover, this 21 foot $(6.405 \mathrm{~m})$ difference in predicted legibility distance comprises only 14 percent of the 150 foot $(45.75 \mathrm{~m})$ interval separating adjacent TES traps used to record vehicle speeds. Consequently, it was not likely that legibility distance could be used to explain any measured differences in either detection rates or the initial locations of vehicle speed reductions.

The other independent variable of the field study was level of visual complexity. Complexity was varied by selecting three matched data collection sites that varied primarily in terms of the visual environment adjacent to the roadway. The visual complexity of each site was determined by first rating the site on each of the visual dimensions of complexity defined in the laboratory study and then using these coded variable values as input to one of the regression equations.

The procedure used to rate the visual dimensions of complexity was the same as that used in the lab study. Specifically, a $35 \mathrm{~mm}$ color slide of each site was taken from a location 400 feet $(122 \mathrm{~m})$ upstream of the medium luminance target sign. In taking these photographs, the camera was aimed straight ahead and was situated inside of a 1976 Ford van at the driver's eye position for a passenger car travelling in the shoulder lane. Each of these slides was projected onto a glassbeaded screen such that the projected scene measured $5^{\prime} \times 6^{\prime} 8^{\prime \prime}(1.525 \mathrm{~m} \times 1.83 \mathrm{~m}$ $20.32 \mathrm{~cm})$. Each site was then evaluated along each complexity dimension by two trained independent evaluators. Notes taken at the site were used to assist

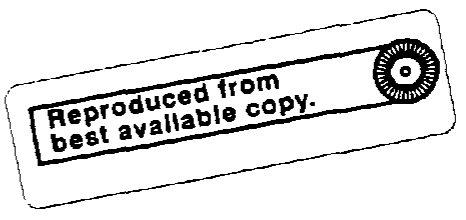


this rating procedure.* Disagreements in initial ratings were resolved by a discussion of the rationales used by each of the evaluators in arriving at their respective ratings. This process was continued until the evaluators reached agreement. The ratings for the complexity variables at each site are presented in Appendix F.

After the complexity variables were rated, the level of complexity characteristic of each site was determined by using the coded variable values as input to the regression equation developed for the yellow diamond pedestrian crossing sign via the lab study. Table 16 lists, for each site, the coded values for each measure used in the regression equation that assesses complexity without the benefit of any sign-to-surround contrast variables.

\section{Dependent Variables}

Two dependent variables, incidence and location of speed reduction were derived by evaluating the speed profile of each subject vehicle as it traversed the 1200 foot $(366 \mathrm{~m})$ measured course. It would have been possible to base this speed profile on either spot speeds taken at each of the nine TES traps or on eight average speeds over each of the 150 foot $(45.75 \mathrm{~m})$ intervals between adjacent traps. The latter method was selected because the interval speeds were judged to be less sensitive to minor, random fluctuations in a vehicle's speed profile. Eight interval speeds, obtained from nine TES traps, comprised the raw data that were used for both of the dependent measures. Given these speed profiles for each subject vehicle, the

Table 15. Specific Luminances* and Predicted Legibility Distances of Target Signs

\begin{tabular}{|c|c|c|c|}
\hline $\begin{array}{l}\text { Level } \\
\text { of } \\
\text { Sign Luminance }\end{array}$ & $\begin{array}{l}\text { FHWA minimum } \\
\text { specific } \\
\text { luminance re- } \\
\text { quirements for } \\
\text { yellow sheeting } \\
\left.\text { (cd/ft-c/tet }{ }^{2}\right)\end{array}$ & $\begin{array}{l}\text { Average } \\
\text { specific } \\
\text { luminance of } \\
\text { target sign } \\
\left(c a / f t-c / f t^{2}\right)\end{array}$ & $\begin{array}{l}\text { Predicted } \\
\text { legibility } \\
\text { distance } \\
\text { (ft/in.letter ht.) }\end{array}$ \\
\hline $\begin{array}{l}\text { low } \\
\star \star \star \\
\text { degraded } \\
\text { Type I I } \\
\text { sheeting }\end{array}$ & 25 & 33 & 45 \\
\hline $\begin{array}{l}\text { medium } \\
\star \star \star \\
\text { new } \\
\text { Type I I } \\
\text { sheeting }\end{array}$ & 50 & 55 & 47 \\
\hline $\begin{array}{c}\text { high } \\
\star \star \star \\
\text { new } \\
\text { Type I I } \\
\text { sheeting }\end{array}$ & 170 & 181 & 48 \\
\hline
\end{tabular}

* Because the subject arivers in the field study responded to the target sign as it appeared in the real-world and because the $35 \mathrm{~mm}$ slides of the sites constituted a less-than-perfect reproduction of the real-world, the site notes were usea in favor of the photographic reproductions whenever there were aiscrepencies between the two sources of information. In general, the slides were charactecized by three types of distortions: (1) a compression of the range of luminances that actually existed at the sites, (2) bloom or halation of bright sources of light resulting froin their overexposure relative to darker elements in the roadway scene, and ( 3 ) a loss of detail especially in those scene elements at the extreme ends of the luminance range. These distortions, together with the loss of dynamic effects, are indicative of. the major difficulties in the use of a static photographic image to evaluate the visual complexity of a real-world dynamic situation.

* All specific luminance values assume an entrance angle of $-4^{\circ}$ and an observation angle of $0.2^{\circ}$. 
Table 16. Regression Equation for Visual Complexity

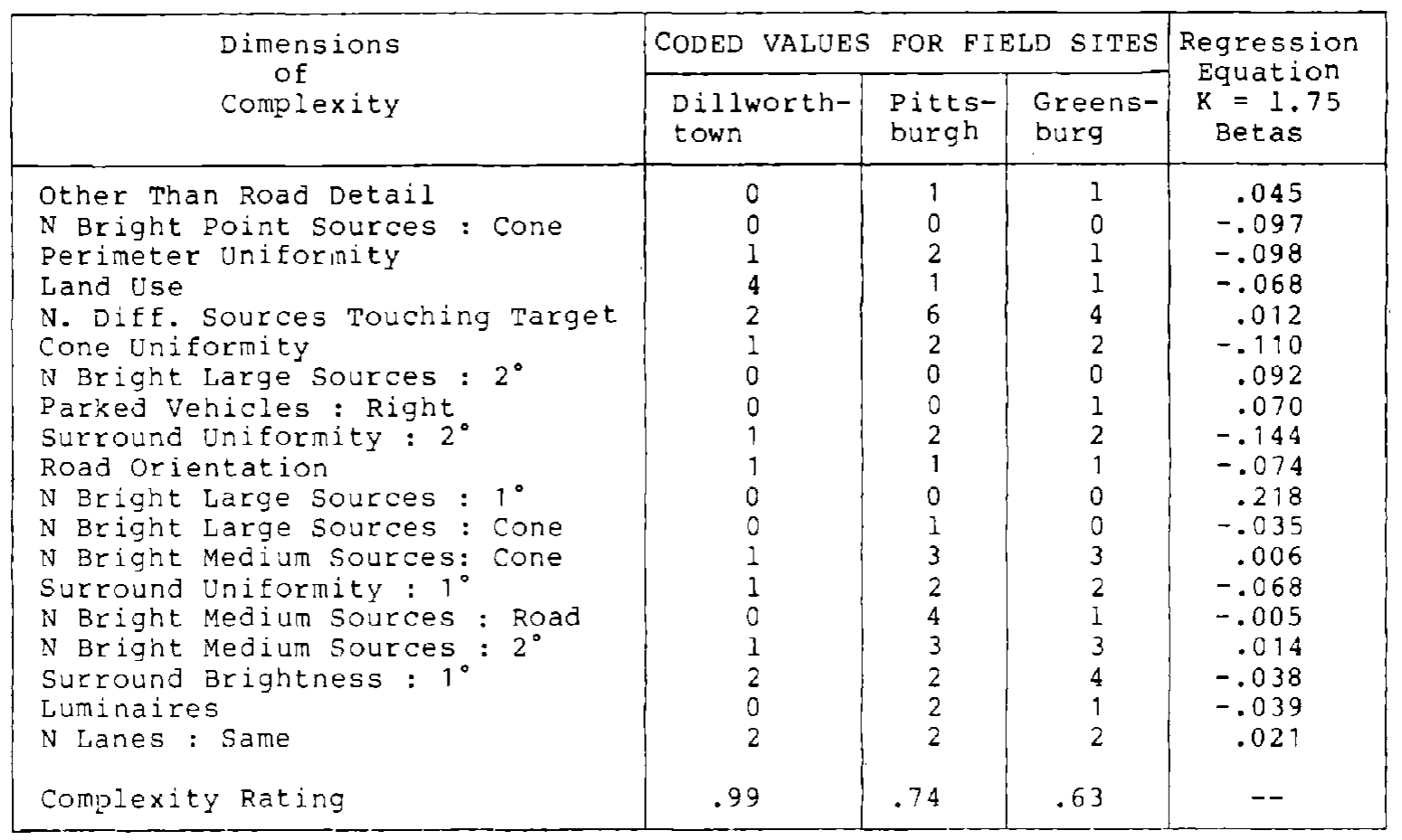

two dependent measures, incidence and location of vehicle deceleration, were computed.

The first dependent measure was the incidence of vehicle decelerations. speed reductions, of course, were assumed to be indicative of sign recognitions. Several alternative measures of speed reduction were considered:

- decelerations to a speed less than the speed limit plus $5 \mathrm{mph}(8 \mathrm{~km} / \mathrm{hr})$,

- decelerations, greater than sone criterion magnitude, defined by the difference between the highest speed in the driver's profile minus the lowest subseguent speed,

- decelerations, greater than some criterion magnitude, defined by the entry speed (average speed between traps one and two) minus exit speed (average speed between traps eight and nine).

All three alternatives were susceptible to two types of errors: excluding vehicles whose drivers did respond to the sign (Ealse negatives) and including vebicles whose drivers did not respond to the sign (falise positives). Such errors ace natural with a surrogate measure. The first of these alternatives seemed most likely to result in a high false negative rate and the second seemed most likely to result in a high false positive rate. On an a priori basis we preferred the third alternative which was independent of the magnitude and location of speed reduction. Location was to be analyzed separately and selecting $5 \mathrm{mph}$ or any other quantity for a speed reduction indicating sign recognition seemed arbitrary. Some preliminary analysis of all three alternatives gave empirical support to the selection of the third alternative as the means of defining deceleration. For within site analyses, vehicles were classified as decelerating when the amount of deceleration (entry speed - exit speed) was greater than zero. In the between site analysis of visual complexity the amount of deceleration was compared to the median deceleration for the control data of the site. The two measures may be expressed as follows:

$$
\begin{array}{ll}
\text { Vehicle decelerated if } & N>0 \\
\text { Vehicle decelerated if } & N>M_{i j}
\end{array}
$$

where: $N=$ Entry speed - exit speed and: $\quad M_{i j}=$ Median (entry speed -

$$
\text { exit speed) Eor site } i, \text { lane } j \text {. }
$$

The second dependent variable was included to provide information indicating

* Developed from the laboratory study data using the Pedestrian Crossing Target. 
whether sign luminance and/or visual complexity influenced the location of the initial vehicle deceleration. A speed reduction location was defined as the first trap at which a measured speed reduction equalled or exceeded that vehicle's mean speed reduction. Only speed reductions after the vehicle's maximum speed was achieved were considered. This method reflected the desire to locate meaningful, intentional speed reductions rather than random fluctuations. Since this measure was also a surrogate for location of sign recognition, it was applied only to those subject vehicles which demonstrated a speed reduction according to the criterion described above. The measure was derived by subjective assessment and an examination of a sample of vehicle speed profiles.

\section{Experimental Design}

The field study was designed to assess the main and interactive effects of sign luminance and visual complexity on both the probability and location of sign recognition. This paradigm was intended to provide information relevant to specific hypotheses stating that both the probability and distance of sign recognition increase as sign luminance increases and as visual complexity decreases. In addition, this design also allowed for an exploration of the potential interactive effects of luminance and complexity on both the probability and location of sign recognition. These hypotheses were examined by recording data indicating the incidence and location of vehicle speed reductions elicited by the SPEED TRAP sign. The percentage and location of these vehicle decelerations were assumed to be indicative of the probability and location of target sign recognition, respectively.

Level of visual complexity was controlled by selecting three highway sites that were matched to the extent possible, except with regard to the nature of the adjacent visual environment, which differed systematically to provide low, medium, and high complexity conditions. As such, visual complexity was a betweensite variable with only one site representing each of three levels of complexity. Because this design utilized only one instance of each complexity level to assess the effect of complexity, any other between-site differences may have confounded the influence of complexity. For this reason, any conclusions about this independent variable should be interpreted cautiously.

Level of sign Iuminance, however, was a within-site variable. Each of three luminance conditions - low, medium, and high - was presented at each site. The target sign was deployed on two nights per site, and the three luminance conditions were balanced across nights to preclude confounding with day of the week. Data were collected for approximately six hours a night, and an attempt was made to divide this period into two or more equal blocks of time. Each of the three luminance conditions was presented for equal intervals of time within each of these blocks, and the order of presentation of the three levels of luminance was counterbalanced across blocks to avoid confounding luminance effects with time of the night. Because of problems due to both inclement weather and hardware malfunctions, it was not possible to keep the blocks of time at a constant duration across nights or to precisely match the start-times of comparable blocks across nights. However, when the duration or the start-times of the blocks could not be matched, luminance conditions were presented such that confounding with either day of the week or time of the night was minimized. The actual experimental design that was followed during data collection is presented in Table 17. The first column under each site in this table indicates the hours during which control data were collected. These times were intended to correspond closely to the hours of data collection under sign conditions so that the control and treatment speed profiles would not be differentially affected by time of night. This matching between the hours of control and treatment data collection was effectively accomplished except at the high complexity site where equipment problems interfered with the first two hours of control-night data collection.

\section{FINDINGS \\ 1. Incidence of Speed Reductions}

One of the major areas of inquiry was the affect of the SPEED TRAP sign on the incidence of speed reductions. The initial analysis was simply to determine if the presence of the SPEED TRAP sign was conducive to speed reductions. In this analysis, scene complexity and sign brightness were ignored; that is, the data were collapsed over the levels of complexity and brightness. The results are given in Table 18 for each of the two travel lanes. (Lane 1 refers to the rightmost or shoulder lane.) In this analysis, treatment data (data collected with the sign present) were compared to control data (those collected with the sign removed) so that the focus was on the effect of the sign. 
Table 17. Experimental Design for Field Data Collection: sign Luminance Conditions Balanced Across

Nights and Times

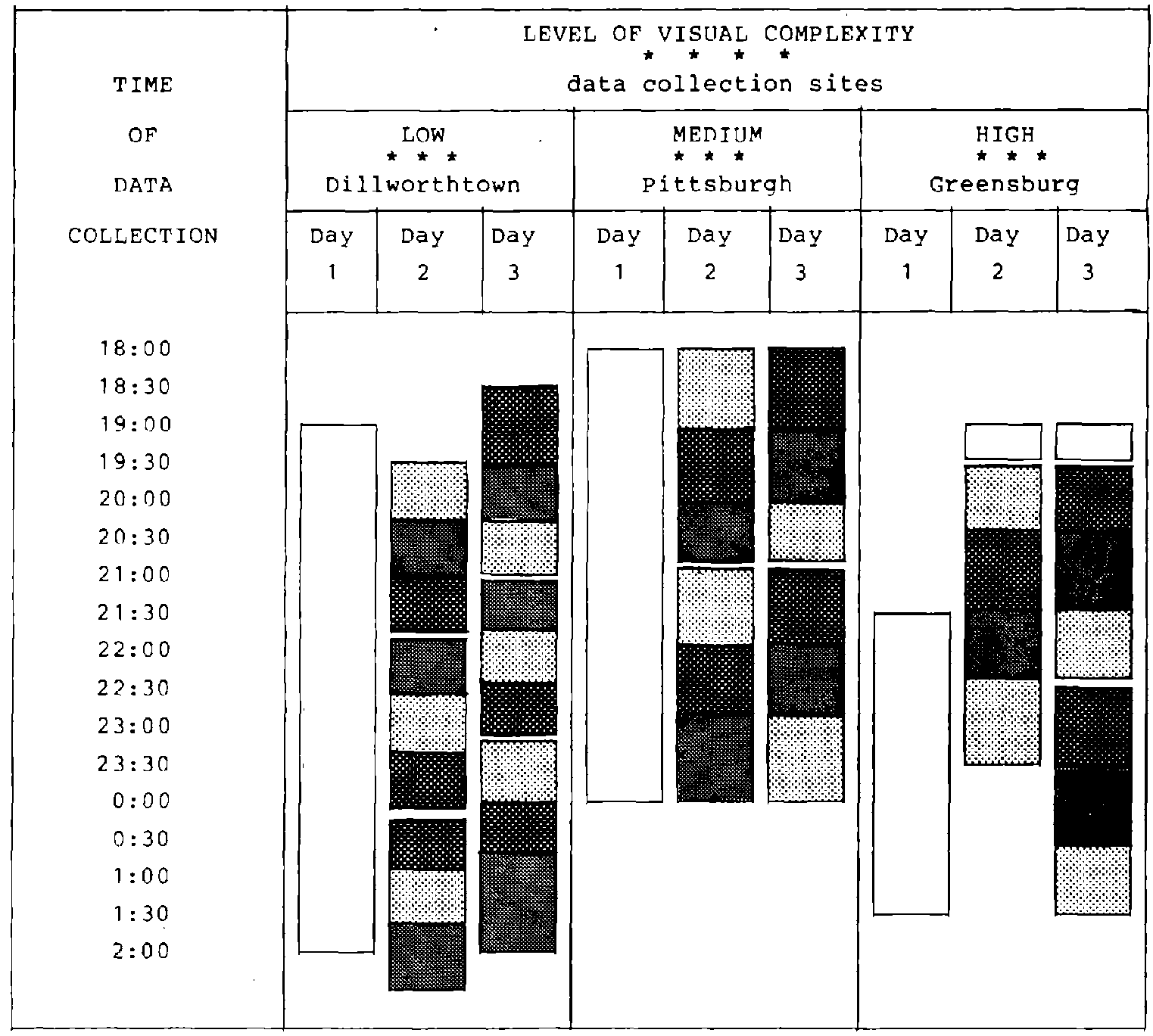

\begin{tabular}{c} 
KEY FOR SIGN LUMINANCE LFVEL \\
\hline$=$ no sign $=$ low $=$ migh
\end{tabular}

N. . A space separating luminance conditions indicates a division between two time-blocks within which each of the sian luminance conditions were presented. 
Table 18. Overall Sign Effects

\begin{tabular}{|c|c|c|c|c|c|}
\hline \multirow[b]{2}{*}{ Lane } & \multirow{2}{*}{$\begin{array}{c}\text { Sign } \\
\text { Present? }\end{array}$} & \multicolumn{3}{|c|}{ Speed Reduction? } & \multirow[b]{2}{*}{ Chi-square } \\
\hline & & Yes & № & 8 (Yes) & \\
\hline \multirow[t]{2}{*}{1} & $\begin{array}{l}\text { Yes } \\
\text { No }\end{array}$ & $\begin{array}{r}440 \\
232 \\
\end{array}$ & $\begin{array}{r}492 \\
308 \\
\end{array}$ & $\begin{array}{l}47.2 \\
43.0\end{array}$ & \\
\hline & Total & 672 & 800 & & 2.49 (NS)* \\
\hline \multirow[t]{2}{*}{2} & $\begin{array}{l}\text { Yes } \\
\text { No }\end{array}$ & $\begin{array}{l}509 \\
246 \\
\end{array}$ & $\begin{array}{r}791 \\
444 \\
\end{array}$ & $\begin{array}{l}39.2 \\
37.5\end{array}$ & \\
\hline & Total & 755 & 1235 & & 2.35 (NS) \\
\hline
\end{tabular}

The chi-square was selected instead of an Analysis of variance (ANOVA) for two reasons. First, since we had a priori hypotheses about the direction of the effect of experimental treatments, a one sided test was considered to have more power and be sensitive to order of treatment levels. Second, since we were dealing with a surrogate measure for a dichotomous event (sign recognition), the ANOVA would have presented interpretive problems.

The results in Table $18 \mathrm{fail}$ to show statistically significant sign effects for either lane. That is, while the proportion of vehicles reducing speed was higher when the SPEED TRAP sign was in place, the magnitude of the increase was not sufficiently large as to preclude the results being due to chance alone.

This was at first quite disappointing since if the sign itself had no meaningful effect, the experiment might have little chance of detecting the effects of sign brightness. However, a second analysis was performed in which the sign effect was examined within data collection sites (i.e. within levels of complexity). In this way, control data (i.e. no-sign data) were better matched with the treatment data and the interactive effects of visual complexity were removed.

Table 19 shows that the sign had an observable effect on lane 1 at the low and the medium complexity sites. In both instances, the sign had the effect of increasing the relative frequency of speed reductions by 9 percent. In other terms, the likelihood of a speed reduction was increased by 33 percent ( 1 $37.2 / 27.9)$ at the low complexity site and by 23 percent $(1-47.7 / 38.8)$ at the medium complexity site.

None of the remaining comparisons in Table 19 was significant. We can only speculate on the absence of statistically significant findings for lane 2 traffic. It may have been due to the greater viewing angle or a difference in the nature or motivations of the drivers who chose to travel in lane 2 .

\section{Visual Complexity}

The findings for lane 1 in Table 19 suggest that there may have been a complexity effect; that is, the sign became less effective as scene complexity increased. This is indicated by the greater likelihood of speed reductions associated with the presence of the sign at the low complexity site and the absence of such an effect at the high complexity site. This effect was tested with data adjusted for the median speed reduction under control conditions. The analysis of these data are presented in Table 20 . The chi-square for lane 1 was significant with the decelerations for the high complexity site being close to a chance prediction of 50 percent. The percent decelerations for the median and low complexity site were 8.7 and 10.7 percent above chance.

The data in Table 19 also show that for any combination of complexity and sign presence there was a lower incidence of deceleration in lane 2 than in lane 1.

\footnotetext{
* All statistical testing was performed with an alpha level of .05. All testing was based on one-sided hypotheses; that is, the null hypothesis was tested against the one-sided alternative hypothesis that the incidence of speed reductions increased due to the presence of the sign or due to increasing sign brightness. Following these conventions, all tabulated test statistics are followed by an $s$ or Ns, respectively, indicating statistical significance or the lack thereof.
} 
Table 19. Sign Effects within Complexity Levels

\begin{tabular}{|c|c|c|c|c|c|c|}
\hline \multirow[b]{2}{*}{ Lane } & \multirow[b]{2}{*}{ Complexity } & \multirow{2}{*}{$\begin{array}{c}\text { Sign } \\
\text { Present? }\end{array}$} & \multicolumn{3}{|c|}{ Speed Reduction? } & \multirow[b]{2}{*}{ Chi-square } \\
\hline & & & Yes & No & 8(Yes) & \\
\hline \multirow[t]{6}{*}{1} & Low & $\begin{array}{l}\text { Yes } \\
\text { No }\end{array}$ & $\begin{array}{r}136 \\
55 \\
\end{array}$ & $\begin{array}{l}230 \\
142 \\
\end{array}$ & $\begin{array}{l}37.2 \\
27.9\end{array}$ & \\
\hline & & Total & 191 & 372 & & 4.88 (S) \\
\hline & Medium & $\begin{array}{l}\text { Yes } \\
\text { No }\end{array}$ & $\begin{array}{r}186 \\
81 \\
\end{array}$ & $\begin{array}{l}204 \\
128 \\
\end{array}$ & $\begin{array}{l}47.7 \\
38.8\end{array}$ & \\
\hline & & Total & 267 & 332 & & 4.40 (S) \\
\hline & High & $\begin{array}{l}\text { Yes } \\
\text { No }\end{array}$ & $\begin{array}{r}118 \\
96\end{array}$ & $\begin{array}{l}58 \\
38\end{array}$ & $\begin{array}{l}67.0 \\
71.6\end{array}$ & \\
\hline & & Total & 214 & 96 & & 0.75 (NS) \\
\hline \multirow[t]{6}{*}{2} & Low & $\begin{array}{l}\text { Yes } \\
\text { No }\end{array}$ & $\begin{array}{l}68 \\
48 \\
\end{array}$ & $\begin{array}{l}354 \\
206 \\
\end{array}$ & $\begin{array}{l}16.1 \\
18.9\end{array}$ & \\
\hline & & Total & 116 & 560 & & 0.86 (NS) \\
\hline & Medium & $\begin{array}{l}\text { Yes } \\
\text { No }\end{array}$ & $\begin{array}{l}230 \\
107 \\
\end{array}$ & $\begin{array}{l}306 \\
179 \\
\end{array}$ & $\begin{array}{l}42.9 \\
37.4\end{array}$ & \\
\hline & & Total & 337 & 485 & & 2.33 (NS) \\
\hline & High & $\begin{array}{l}\text { Yes } \\
\text { No }\end{array}$ & $\begin{array}{r}211 \\
91 \\
\end{array}$ & $\begin{array}{r}131 \\
59 \\
\end{array}$ & $\begin{array}{l}61.7 \\
60.7\end{array}$ & \\
\hline & & Total & 302 & 190 & & 0.05 (NS) \\
\hline
\end{tabular}

Table 20. Complexity Effects on Adjusted Speed Reductions

\begin{tabular}{|c|c|c|c|c|c|}
\hline \multirow[b]{2}{*}{ Lane } & \multirow[b]{2}{*}{ Complexity } & \multicolumn{3}{|c|}{$\begin{array}{c}\text { Adjusted } \\
\text { Speed Reduction? }\end{array}$} & \multirow{2}{*}{$\begin{array}{c}\text { Chi-Square } \\
\text { (High vs. Low) } \\
\end{array}$} \\
\hline & & Yes & $\underline{\text { No }}$ & $8($ Yes $)$ & \\
\hline \multirow[t]{2}{*}{1} & $\begin{array}{l}\text { Low } \\
\text { Medium } \\
\text { High }\end{array}$ & $\begin{array}{r}222 \\
229 \\
87 \\
\end{array}$ & $\begin{array}{r}144 \\
161 \\
89 \\
\end{array}$ & $\begin{array}{l}60.7 \\
58.7 \\
49.4\end{array}$ & \\
\hline & Total & 538 & 384 & t & 6.41 on 2 df (s) \\
\hline 2 & $\begin{array}{l}\text { Low } \\
\text { Medium } \\
\text { High }\end{array}$ & $\begin{array}{l}208 \\
294 \\
164 \\
\end{array}$ & $\begin{array}{l}214 \\
242 \\
178 \\
\end{array}$ & $\begin{array}{l}49.3 \\
54.9 \\
48.0\end{array}$ & \\
\hline & Total & 666 & 634 & & 0.13 on 2 df (NS) \\
\hline
\end{tabular}


In other words, lane 2 vehicles were more likely to be accelerating than were vehicles in lane 1. While one might expect higher speeds in lane 2, this alone cannot explain the more frequent accelerations.

The most plausible explanation hinges on the fact that as shown in Table 14, each of the sites was preceded by a traffic signal. Because of this, it was reasonable that not all drivers had accelerated to their desired speed prior to entering the test course and that this was more likely to be true for drivers having the higher desired speeds. Since drivers with higher desired speeds would be more 1 ikely to be in lane 2 , one could expect to observe a greater frequency of accelerations in that lane.

Further examination of Table 19 indicates that the incidence of speed reductions was greater for the high complexity site than for the low complexity site. Since this was true not only when the sign was present but also when it was not, this behavior can probably best be explained on the basis of factors relating to site rather than sign characteristics.*

\section{Sign Brightness}

The next analysis was performed to determine if sign brightness influenced the likelihood of speed reductions. Specifically, did brighter signs elicit more frequent speed reductions? The results are shown in Tables 21 and 22 where the effect of sign brightness is examined within each complexity level.

It might be noted that in this analysis there was no need for the control data since the use thereof would provide equal adjustments to brightness effects within any given site or complexity level.

Thus, for within-site comparisons of the type used here, such adjustments would cancel out and serve no useful purpose.

As noted earlier, there were no significant effects for the lane 2 data. For lane 1 , only the medium complexity data reflected a statistically significant sign brightness effect. (The test statistics resulted from a comparison of the high versus the low brightness sign; while this procedure failed to utilize all the available information, it allowed the testing of one-sided hypotheses thereby reflecting the ordinal nature of the levels of sign brightness.)

Once again, plausible, but speculative, explanations for the lane, findings are offered in.the following. First, at the low complexity site, the low brightness sign may have been sufficiently detectable that increased brightness was superfluous; hence, increased brightness would not be expected to yield beneficial effects. The medium complexity site may have fallen in a range of reduced sign conspicuity where sign detection was sensitive to sign brightness in the expected way. Finally, the high complexity site differed from the other two in a unique way. On-site observations by the experimenters revealed that the sign was markedly darker than its surround. As a result, increasing sign brightness resulted in a reduction of sign-surround contrast. This, in turn, could well have led to the observed, though statistically insignificant, reduction in driver responsiveness to increases in sign brightness as shown in Table 21 .

\section{Location of speed Reductions}

In addition to the incidence of speed reductions, the locations of the speed reductions were also analyzed. Primary interest was in whether increased brightness yielded earlier speed reductions; hence, one-sided hypotheses were used. The results for lane 1 are in Table 23 . As before, tests of sign effects involved comparing the overall sign results to nosign results, and brightness effects were studied by comparing the low brightness to high brightness signs; all tests were conducted within complexity levels. In all instances, t-tests were used. In no instance was there a statistically

* As indicated above, upstream traffic signals could have been conducive to a surplus of accelerating vehicles within each of the test sites. However, while roadway characteristics were quite consistent before, in, and after the test site for the low and medium complexity locations, this was not true for the high complexity site where two factors may have come into play. First, there was a slight crest vertical curve approxomately 200 feet beyond the location of the test sign. This had the effect of limiting downstream sight distance and may have contributed to reduced accelerations. second, approximately 350 feet after the end of the measured course was a $Y$-intersection with through traffic going to the left. This may have been conducive to an increase in traffic turbulence and, hence, increased deceleration frequencies. Note that although subject vehicles were selected to have large headways, no restriction was placed on the presence of vehicles in the adjacent lane. Hence, the upstream choice point could well have produced a need for lower speeds and, as a result, an increased incidence of speed reductions. 
Table 21. Brightness Effects Within Complexity Levels - Lane 1

\begin{tabular}{|c|c|c|c|c|c|}
\hline \multirow[b]{2}{*}{ Complexity } & \multirow[b]{2}{*}{ Brightness } & \multicolumn{3}{|c|}{ Speed Reductior? } & Chi-Square \\
\hline & & Yes & No & $8($ Yes $)$ & (High vs. Low) \\
\hline \multirow[t]{2}{*}{ Low } & $\begin{array}{l}\text { Low } \\
\text { Medium } \\
\text { High }\end{array}$ & $\begin{array}{l}45 \\
37 \\
54 \\
\end{array}$ & $\begin{array}{l}76 \\
67 \\
87 \\
\end{array}$ & $\begin{array}{l}37.2 \\
35.6 \\
38.3\end{array}$ & \\
\hline & Total & 136 & 230 & & 0.03 (NS) \\
\hline \multirow[t]{2}{*}{ Medium } & $\begin{array}{l}\text { Low } \\
\text { Medium } \\
\text { High }\end{array}$ & $\begin{array}{l}49 \\
59 \\
78 \\
\end{array}$ & $\begin{array}{l}67 \\
72 \\
65 \\
\end{array}$ & $\begin{array}{l}42.2 \\
45.0 \\
54.5\end{array}$ & \\
\hline & Total & 186 & 204 & & 3.88 (S) \\
\hline \multirow[t]{2}{*}{$\mathrm{High}$} & $\begin{array}{l}\text { Low } \\
\text { Medium } \\
\text { High. }\end{array}$ & $\begin{array}{l}19 \\
44 \\
55 \\
\end{array}$ & $\begin{array}{r}7 \\
20 \\
31 \\
\end{array}$ & $\begin{array}{l}73.1 \\
68.8 \\
64.0\end{array}$ & \\
\hline & Total & 118 & 58 & & 0.74 (NS) \\
\hline
\end{tabular}

Table 22. Brightness Effects within Complexity Levels - Lane 2

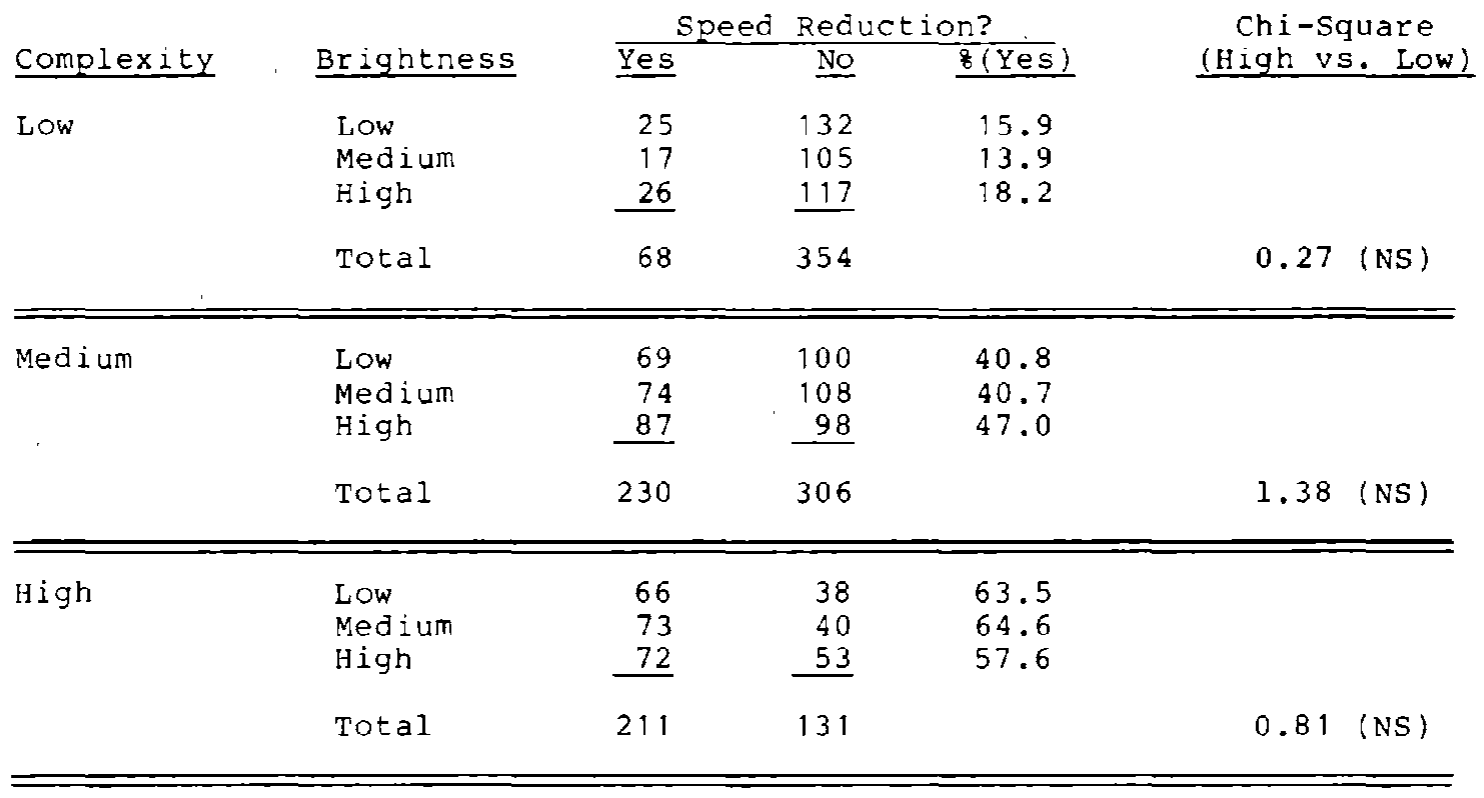


Table 23. Sumary statistics For Location of Speed Reduction - Lane 1

\begin{tabular}{|c|c|c|c|c|c|}
\hline Complexity & Brightness & $\underline{\mathbf{N}}$ & $\begin{array}{c}\text { Mean } \\
\text { Trap } \\
\text { Location } \\
\end{array}$ & $\begin{array}{c}\text { Mean } \\
\mathrm{ft.} \text { from } \\
\text { sign } \\
\end{array}$ & $\begin{array}{l}\text { Standard } \\
\text { Deviation }\end{array}$ \\
\hline \multirow[t]{3}{*}{ Low } & $\begin{array}{l}\text { Low } \\
\text { Medium } \\
\text { High }\end{array}$ & $\begin{array}{l}45 \\
37 \\
54 \\
\end{array}$ & $\begin{array}{l}4.40 \\
5.00 \\
4.39 \\
\end{array}$ & $\begin{array}{r}-90 \\
0 \\
-\quad 92 \\
\end{array}$ & $\begin{array}{l}1.93 \\
1.41 \\
1.65 \\
\end{array}$ \\
\hline & Overall & 136 & 4.56 & -66 & 1.71 \\
\hline & No Sign & 55 & 4.56 & -66 & 1.90 \\
\hline \multirow[t]{3}{*}{ Medium } & $\begin{array}{l}\text { Low } \\
\text { Medium } \\
\text { High }\end{array}$ & $\begin{array}{l}49 \\
59 \\
78 \\
\end{array}$ & $\begin{array}{l}4.59 \\
4.90 \\
5.13 \\
\end{array}$ & $\begin{array}{l}-76 \\
-\quad 15 \\
+\quad 20 \\
\end{array}$ & $\begin{array}{l}1.77 \\
1.77 \\
1.92 \\
\end{array}$ \\
\hline & Overall & 186 & 4.91 & -14 & 1.85 \\
\hline & No Sign & 81 & 4.46 & -81 & 1.85 \\
\hline \multirow[t]{3}{*}{ High } & $\begin{array}{l}\text { Low } \\
\text { Medium } \\
\text { High }\end{array}$ & $\begin{array}{l}19 \\
44 \\
55 \\
\end{array}$ & $\begin{array}{l}4.63 \\
4.30 \\
4.24 \\
\end{array}$ & $\begin{array}{r}-56 \\
-105 \\
-114 \\
\end{array}$ & $\begin{array}{l}1.75 \\
1.63 \\
1.63 \\
\end{array}$ \\
\hline & Overall & 186 & 4.91 & -101 & 1.85 \\
\hline & No Sign & 96 & 4.11 & -134 & 1.69 \\
\hline
\end{tabular}

significant effect in which the sign itself or increasing brightness resulted in earlier speed reductions. One noticeable trend in Table 23, however, suggests that at the high complexity site, increasing levels of sign brightness may have facilitated earlier sign recognition to some extent.

\section{CONCLUSIONS}

Because the field study results reflect data collected at only three sites that differed on a laboratory derived scale of visual complexity, any conclusions concerning the main and interactive effects of visual complexity and sign luminance are tentative at best. Nevertheless, the conclusions derived from the field study results are not unexpected and are conceptually tenable. First, the data indicate that, in general, complexity does interfere with the detection-recognition of yellow, diamond-shaped traffic signs. Further, at medium levels of visual complexity, increasing the luminance of such a sign appears to facilitate detectionrecognition of the device. Under conditions of low visual complexity, however, there is no evidence indicating that increasing sign luminance has any measurable effect on driver performance. Levels of sign luminance higher than that provided by new Type I sheeting do not seem to improve the conspicuity of yellow, diamond-shaped traffic signs. The same conclusion is reached concerning high complexity scenes; however, since this situation did not produce a sign effect, the possibility remains that luminances greater than that provided by Type III sheeting (such as a selfiliuminated sign) may be effective.

\section{SUMMARY AND RECOMMENDATIONS}

The two studies reported build a strong case for the usefulness of visual complexity as a predictor of sign detection and recognition. The results indicate that at locations within complex visual scenes, measures of the scene and the sign's surround predict visual performance better than sign contrast and brightness. The laboratory study indicated that target brightress could offset the detrimental effects of visual complexity. Although the field study supported this finding, it also suggested that brightness might not have an effect at the extremes of visual complexity. The results of the field study suggested the theoretical relationships shown in Figure 10. When visual complexity is low, performance is asymptotic and any reasonably reflective sign will be recognized. When visual complexity is 


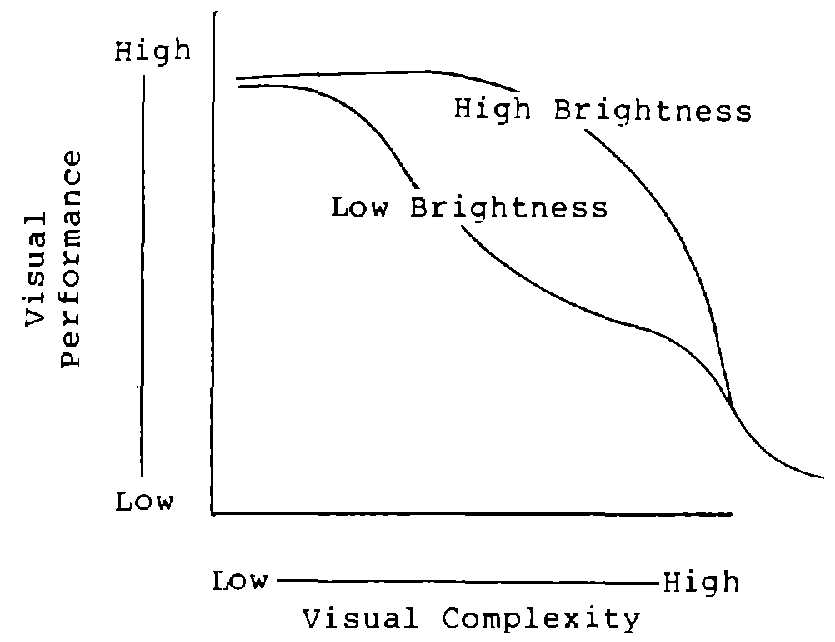

Figure 10. Theoretical Relationship suggested by the field study

extremely high, performance is likely to be equally poor with all retroreflective signs and extraordinary measures such as sign redundancy or internal illumination may be needed.

The measures of visual complexity, sign brightness and contrast should provide a useful contribution to future research. Methods for measuring contrast, surround, and other variables, had not previously been well defined for complex stimuli. The photometric methods for measuring internal contrast should also be of benefit. The fact that visual measures can have significant validity and be reliably coded suggests the potential for a practical method whereby field personnel can judge the visual complexity of a location and its effect on driver recognition of traffic signs.

The absence of cross validation and the inclusion of only three sites in the field study places abvious reservations on the reliability of the findings. Nevertheless, the consistency and pattern of results suggests that continuation of this reseacch seems warranted. Given the abstract nature of most previous

research, this report represents an important step toward developing practical and useable results. Additional work is now needed to refine the measures of $v$ isual complexity; formulate and test hypotheses about their interrelationships; and to develop a scale and procedure which makes visual complexity easier to measure.

The conclusions of both the laboratory and field studies lead to a number of recommendations; some for immediate use by traffic engineers, and others for additional research in the area of yisual complexity.
- Since the black on white regulatory sign is less conspicuous than the other signs tested, it should not be used for providing information which requires long decision sight distances.

- Until the reliability and shape of the complexity curve are known, warning signs, construction signs, and stop signs should have the highest practical luminance at all but low complexity locations, since greater luminance improves detection at medium complexity sites and the cut-off between medium and high complexity cannot yet be determined.

- Less concern need be given to the luminance of regulatory signs since they appear to meet decision sight distance requirements in nón-complex areas and their conspicuity in complex scenes is not improved by increases in luminance.

- Additional analyses and research are necessary to make visual complexity easier to measure. Factor analyses should be applied to determine a set of global complexity factors. Traffic engineers could be asked tc scale a new set of scenes on these factors in both the real world and from photographs. The reliability of the scales could then be assessed and cut-off scores determined for classifying sign locations as high, medium, and low.

- Further research is also needed to determine the effects of selfilluminating signs (where practical), higher contrast from black borders, and sign size on the detection of signs in medium and high complexity areas. 
Baker, C. A., Morris, D. F., \& Steedman, W. C. Target recognition on complex displays. Human Factors, $1960,2(2), 51-61$.

Banks, W. P., Bachrack, K. M., Larson, D. W. The asymmetry of lateral interference in visual filter identification. Perception and Psychophysics. 1977, 22(3), $232-240$.

Banks, W. P., Bodinger, D., \& Illige, M. Visual detection accuracy and target-noise proximity. Bulletin of the Psychonomic Society, $1974, \underline{2}(4 \mathrm{~B}), 411-414$.

Brown, B., Monk, T. H. The effect of local target surround and whole background constraint on visual search times. Human Factors, 1975, 17, 81-88.

Cahill, M. C. \& Carter, R. C. Color code size for searching displays of different density. Human Factors, $1976,18(3), 273-280$.

Compton, S. Night-driving patterns and characteristics of older drivers. Personal communication, 1980 .

Crawford, A. The perception of light signals: the effect of the number of irrelevant lights. Ergonomics, 1962, 5(3), 417-428.

Dornic, S., \& Borg, G. Visual search for simple geometric figures: the effect of target-noise similarity. Reports from the Institute of Applied Psychology, University of Stockholm, $1 \overline{971,22}$.

Elstad, J. O., Fitzpatrick, J. T., \& woltman, H. L. Requisite luminance characteristics for reflective signs. HRB Bulletin 336, 1962, 51-60.

Eriksen, B. A., Eriksen, C. W. Effects of noise letters upon the identification of a target letter in a nonsearch task. Perception and Psychophysics, 1974, 16, $143-149$.

Estes, W. R., \& Wolford, G. L. Effects of spaces on report of tachistoscopically presented letter strings. Psychonomic Science, 1971, 25, 77-80.

Forbes, T. W., Fry, J. P., Joyce, R. P., \& Pain, R. F. Letter and sign contrast, brightness, and size effects on visibility. Highway Research Record, 1968a, 216, $48-54$.

Forbes, T. W., pain, R. F., Fry, J. P., \& Joyce, R. P. Effects of sign position and brightness on seeing simulated highway signs. Highway Research Record, 1967, $164,29-37$.

Forbes, T. W., Pain, R. F., Joyce, R. P., \& Fry, J. P. color and brightness factors in simulated and full-scale traffic sign visibility. Highway Research Record, $1968 b, \underline{216}, 55-65$.

Green, B. F., \& Anderson, L. K. Color coding in a visual search task. Journal of Experimental Psychology, 1956, 51(1), 19-24.

Hanson, D. R., \& Dickson, A. D. Significant visual properties of some fluorescent pigments. Highway Research Record, $1963,49,13-29$.

Jenkins, S. E. Consideration of effects of background on sign conspicuity. Draft paper accepted with minor revisions for 11 th Conference of the Australian Road Research Board.

Lovie, A. D., \& Lovie, P. The effect of mixed visual contrast schedules on detection times for both free and horizontally structured visual search. Ergonomics, 1970 , $13,735-741$.

Mackworth, N. H. Visual noise causes tunnel vision. Psychonomic Science, 1965, 3 , $67-68$. 
McIntyre, C., Fox, R., \& Neale, J. Effects of noise similarity and redundancy on the information processed from brief visual displays. Perception and Psychophysics, $1970, \underline{7}, 328-332$.

Monk, T. H. \& Brown, B. The effect of target surround density on visual search performance. Human Factors, $1975,17,356-360$.

olson, P. L., \& Bernstein, A. Determine the luminous requirements of retroreflective highway signing. Ann Arbor, Mich.: Highway Safety Research Institute, 1977.

Pain, R. Brightness and brightness ratio as factors in the attention value of highway signs. Highway Research Record, 1969, 275, 32-40.

Seguin, E. L., Crowley, K. W., Zweig, W. D., \& Gabel, R. J. Traffic evaluator system users manual (Vol. 1, Overview and field data acquisition guide). U.S. Department of Transportation, Washington, D.C.: Federal Highway Administration, December 1981 , in press.

Shaw, P. Processing of tachistoscope displays with controlled order of characters and spaces. Perception and Psychophysics, 1969, 6 , 257-266.

Smith, S. L. Color coding and visual search. Journal of Experimental Psychology, $1962,64,434-440$.

Smith, S. L., Thomas, D. W. Color versus shape coding in information displays. Journal of Applied Psychology, 1964, 48, 137-146.

Steedman, W. C., \& Baker, C. A. Target size and visual recognition. Human Factors, $1960, \underline{2}, 120-127$.

U. S. Department of Transportation, Federal Highway Administration. Standard Highway Signs. Washington, D.C.: Author, 1979.

U. S. Department of Transportation, Federal Highway Administration. Traffic control devices and delineators. In standard specifications for construction of Roads and Bridges on Federal Highway Projects (Section 633). washington, D.C.: Author, 1979 .

Wolford, G. L., \& Hollingworth, S. Retinal location and string position as important variables in visual information processing. Perception and psychophysics, 1974, $16,437-442$. 


\section{APPENDIX A \\ VISUAL DIMENSIONS OF COMPLEXITY AND CONTRAST}

\section{Evaluation Procedure}

The visual evaluation procedure was carried out by two trained independent raters. This process began with the definition of each candidate complexity factor in terms of observable characteristics. After each variable was defined, visual criteria were specified in order to scale the full range of potential variable values to a practical, finite number. The candidate factors were then used to evaluate the laboratory stimuli. Each scene was first rated along each of the candidate dimensions by two trained independent evaluators. Disagreements between raters were resolved via mutual discussion of the respective rationales that were followed in arriving at the initial rating. This process was continued until the evaluators reached agreement. The practice of comparing ratings also provided a means of reducing clerical errors in the evaluation procedure.

This evaluation procedure was applied to all of the candidate complexity and contrast factors that were not photometric. complexity factors were categorized as either scene or surround variables. Scene variables are those factors that are characteristic of a given roadway scene. Surround variables refer to dimensions immediately adjacent to the target sign; consequently, the values of surround variables vary as a function of the placement of a given sign within each scene. Contrast variables reflect the brightness contrast between a sign and elements of the surround that are contiguous to the target. Contrast variable values are influenced by both sign type and sign placement.

\section{Scene Variables}

Scene variables describe visual characteristics of the roadway scene. Each scene variable was assigned to one of three groups: factors that were characteristic of the entire scene, factors that were evaluated within each of four different spatial areas into which each scene was divided, and factors that applied only to that portion of the scene where the target sign could reasonably be expected to appear.
The first group of scene factors were those characteristic of the entire scene and, as such, were independent of both target type and target placement within the scene. The 720 slides used as stimu$l i$ in the laboratory study were comprised of 80 roadway scenes. Each of the 80 scenes occurred nine times -- once without a target, and with each of two targets at two distances at two levels of brightness. Since the scene variables characteristic of the entire scene were not influenced by target conditions, only one representative of each of the 80 roadway scenes required evaluation along the dimensions of this group of variables. The representative slides that were evaluated were those slides that did not contain any target signs. Table 24 summarizes this group of scene variables. The first column of the table identifies and defines the scene variable. The second column indicates the actual scaled values used to rate each variable, and the third column lists corresponding coded values that were used for analysis.

The final three variables listed in Table 24 (i.e. NUMBER OF LANES: SAME, AREA TYPE, and LAND USE) are roadway characteristics and, as such, are legitimate scene variables. However, in contrast to the other scene variables, these factors do not reflect individual discrete physical dimensions depicted in the laboratory slides. Rather, they are intervening variables whose values can be determined only through a synthesis of several scene elements by the observer. In other words, each of these intervening variables represents a configuration of relevant visual stimuli within each scene.

For several of the variables 1 isted in Table 24 -- as well as for several other complexity factors that will be discussed later - - the actual variable values were determined by means of frequency counts. When these counts were greater than or equal to 9 , the variable value was always coded as 9. This convention was necessary both to reduce data processing time and to simplify the scoring of variables when the frequencies became unusually large. In the entire subjective evaluation procedure, 18 variables were coded in this manner. Of these, four had a score of 9 in more than 10 percent of the scenes, and only two variables had a score of 9 in more than 30 percent of the scenes.* The use of a 9 to code variable

* The specific variables that received a score of 9 for more than $10 \%$ of the stimuli were: (A) N Bright Medium Sources - Left of Cone, (B) N Inits Area: Bright Large Sources - Left of Cone, (C) N Units Area: Bright Large Sources - Right of Cone, and (D) $\mathrm{N}$ Different Surfaces Touching Target. The variables with a score of 9 for more than $30 \%$ of the stimuli were: (A) $N$ Units Area: Bright Large Sources -- On the Road and (B) N Bright Point Sources -- $2^{\circ}$ Surround. 
Table 24. Scene Variables Characteristic of the Entire Roadway Scene $(\mathrm{N}=80)$

\begin{tabular}{|c|c|c|}
\hline $\begin{array}{c}\text { SCENE } \\
\text { VARIABLES }\end{array}$ & $\begin{array}{c}\text { ACTUAL } \\
\text { VARIABLE VALUES }\end{array}$ & $\begin{array}{l}\text { CODED } \\
\text { VARIABLE VALUES }\end{array}$ \\
\hline 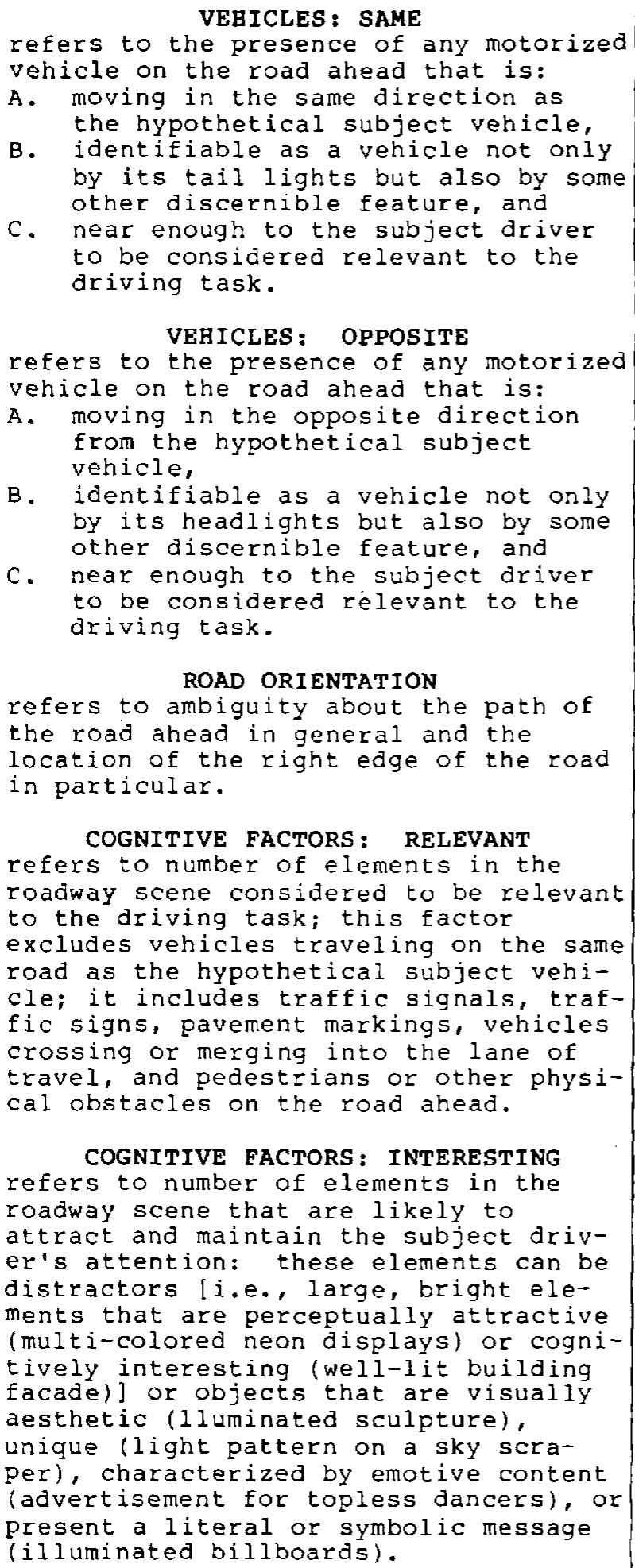 & $\begin{array}{c}\text { ambiguous } \\
\text { clearly indicated }\end{array}$ & $\begin{array}{l}\text { actual number: } 0-8 \\
9 \text { or more: } 9 \\
\text { actual number: } 0-8 \\
9 \text { or more: } 9\end{array}$ \\
\hline
\end{tabular}


Table 24. Scene Variables - Entire Scene (continued)

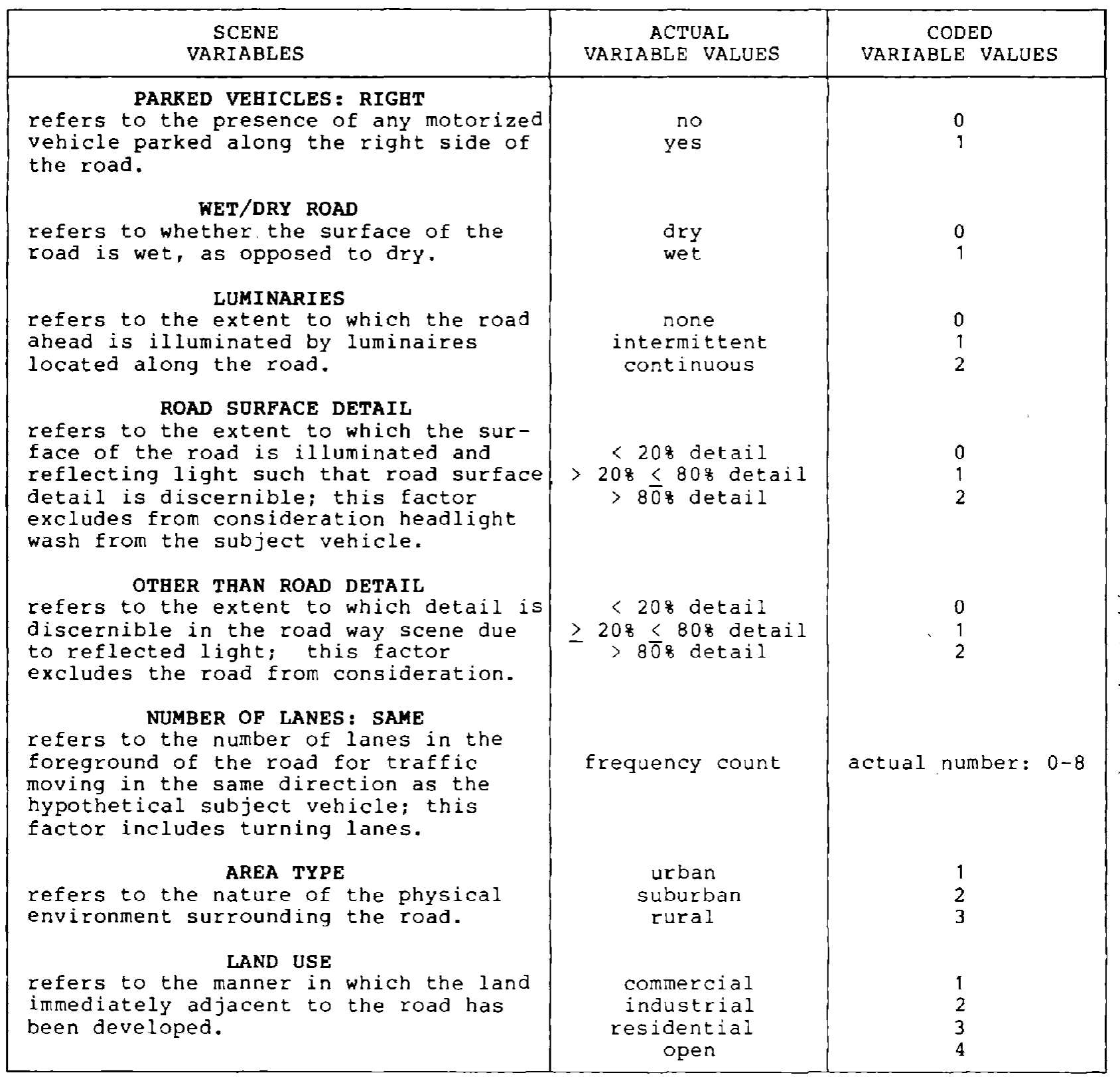

counts greater than or equal to 9 therefore probably did not have any major affect on the measurements recorded.

The second group of scene variables was comprised of those dimensions that were measured in each of four spatial areas or zones into which each roadway scene was divided. The variables evaluated in each of these zones included the number of various-sized sources of bright light. Figure 11 is an abstract representation of these four zones. The first zone is the road itself; this zone includes any sources of bright light that are either on the surface of the road or associated with vehicles on the road. Examples of these light sources include headlights, tail lights, pavement markings, road surface wash from luminaires, and reflections off vehicles. The next zone is that part of the roadway scene which is defined by the possible location of one of the four targets; that is, the area in which shoulder-mounted signs on the right side of the road are typically located. This is a cone-shaped zone that is immediately adjacent and parallel to the right edge of the road. The cone begins about 200 feet $(61 \mathrm{~m})$ downstream from the 
hypothetical subject vehicle and extends to either the vanishing point of the road or to a distance of 850 feet $(259 \mathrm{~m})$ ahead of the subject vehicle, whichever occurs first. In general, the top boundary of the cone is defined by the maximum realistic height of shoulder-mounted traffic signs, approximately 12 feet $(3.7 \mathrm{~m})$. The height of the cone actually varies to some extent from one scene to another, depending upon the height of other traffic signs within the scene. The bottom boundary of the cone usually runs about 4 feet $(1.2 \mathrm{~m})$ above and parallel to the right edge of the road. However, when vehicles are parked along the right side of the road, the cone excludes these vehicles, which are considered to be in the "road" zone. The ultimate criterion for defining the boundaries of the cone within a given scene was determined by searching for shoulder-mounted traffic signs within the scene and then including the area that was scanned in the cone. Examples of bright light sources frequently found within the cone include traffic signals, advertising signs, store-front windows, and illuminated building facades. The final two spatial areas into which scenes were divided are the areas to the left and to the right of the cone of possible target location, excluding the road and the cone itself. The general boundaries of these areas can be determined by exam- ining Figure 11. Examples of bright light sources that are characteristic of these two zones include Iuminaires, building windows, and well-lit service station areas, as well as several of the other scene elements that have already been mentioned.

Among the scene variables evaluated in each of these four zones were the actual number of various-sized, bright sources of light. That is, when a given roadway scene was evaluated, frequency counts of the number of point, medium, and large sources of bright light were made for each of the four spatial areas. Before these scene variables could actually be evaluated, however, it was necessary to establish criteria for defining "point," "medium," "large," and "bright." The criterion used to discriminate among sizes of light sources was the area subtended by the light source when the roadway scene was projected onto the screen in the laboratory; Table 25 illustrates these areas. The first column of the table identifies the size of the light source, and the second column specifies the associated areas on the projection screen, expressed in square inches. The third column of the table delineates the corresponding visual angles subtended for the subject drivers by point-, medium-, and large-sized light sources that are circular in shape.

\begin{tabular}{|c|c|}
\hline \multicolumn{2}{|c|}{ KEY } \\
\hline ROAD \\
\hline $\begin{array}{c}\text { CONE OF POSSIBLE } \\
\text { TARGET LOCATION }\end{array}$ \\
\hline AREA LEFT \\
OF THE CONE \\
\hline AREA RIGHT \\
OF THE GONE
\end{tabular}

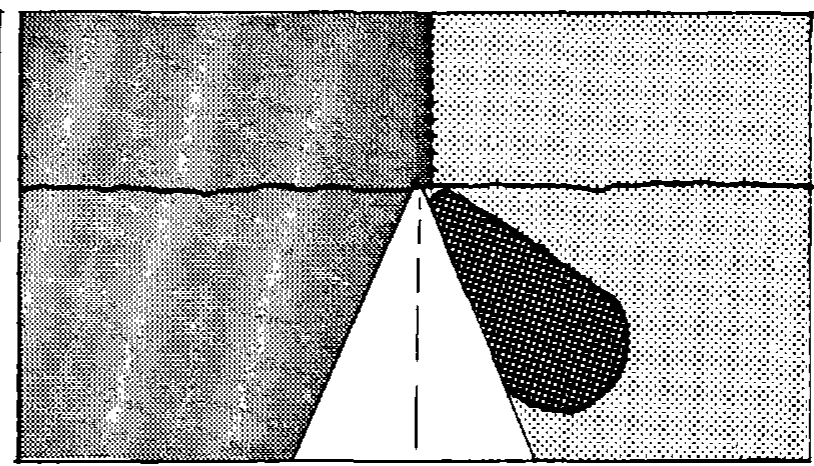

Figure 11. Four Spatial Areas or zones of a Roadway Scene

Table 25. Criteria for "Point," "Medium," and "Large" Light Sources

\begin{tabular}{|l|c|c|}
\hline $\begin{array}{c}\text { TYPE OF } \\
\text { LIGHT SOURCE }\end{array}$ & $\begin{array}{c}\text { SIZE CRITERIA } \\
\text { IN }\end{array}$ & $\begin{array}{c}\text { VISUAL ANGLE } \\
\text { SUBTENDED BY } \\
\text { CIRCULAR SOURCES }\end{array}$ \\
\hline $\begin{array}{l}\text { SOUARE INCHES } \\
\text { medium }\end{array}$ & $\leq 0.44$ & $<18^{\prime \prime}$ \\
large & $0.44<\mathrm{A}<9.0$ & $18^{\prime \prime} \leq$ VA $\leq 1^{\circ} 8^{\prime \prime}$ \\
\hline
\end{tabular}


The criterion that was used to differentiate "bright" sources of light from all other sources involved the use of a twolens polarizing system. Specifically, two $58 \mathrm{~mm}$ Hoya polarizing lenses were mounted onto the front of the lens of the Besseler slide projector that was used to project the roadway scenes. One of the polarizing lenses was then rotated in order to reduce the amount of light passing through the polarizing system to the absolute minimum possible. The effect of this was to reduce most scene detail to a uniform dark shadow, leaving only the brightest scene elements barely discernible. In photometric terms, the effect of this procedure was to reduce the luminance of the brightest light sources by a factor of four log units. For example, luminaires, vehicle headlamps, and selfilluminated advertising signs were generally the brightest light sources in the roadway scenes. The unfiltered luminance of these elements was approximately 70 foot-Lamberts. However, when the polarizing system was implemented, the luminance of these same elements was approximately 0.007 foot-Lamberts.

The scene variables evaluated in the defined spatial areas of the roadway scenes are listed in Table 26 . The first four variables listed, which utilize the size and brightness criteria just described, were measured in each of the four zones of the laboratory scenes. The remaining two variables (CONE UNIFORMITY and CONE BRIGHTNESS) were assessed only in one of the zones -- the cone of possible target location. The variable values that describe the level of cone Brightness (as well as level of surround Brightness and perimeter Brightness) have been defined in as concrete a manner as possible. "Dark" refers to spatial areas that are generally gray or black with few sources of light. Examples of dark areas include telephone poles, shadows, and the night sky. A "slight" level of illumination means that the area being evaluated is primarily comprised of reflected light, with occasional sources of light that are moderately bright and perhaps a few intense sources. Scene elements that were rated as slight were generally reflecting light from an external source, such as building facades or patches of road surface that are illuminated. A "moderate" level of brightness describes a region containing mostly highly reflective items or self illuminating objects that are moderately bright. some examples of moderately bright sources of light were bright biliboards and well 1 it store-fronts. An "intense" level of illumination describes a region where there are frequent occurrences and large areas of intense light sources. Tn the projected slides, these sources were always self illuminating objects near the high end of the film such that color and fine detail were generally not discernible. Examples of these sources of light included luminaires, headlights, and bright internally illuminated commercial signs.

None of the variables Iisted in Table 26 was affected by either target type or target location within the scene. Because of this, only one slide representing each of the 80 roadway scenes required evaluation along the dimensions of these variables. Once again, the slides that were evaluated along the dimensions of the variables listed in Table 26 were the no-target scenes.

The final group of scene variables consists of those dimensions that were measured within the cone of possible target location only. These variables can be described as stimuli that could be confused with the target signs. These variables are isted in Table 27. The latter two variables 1 isted in this table refer to traffic signs and scene elements, respectively, whose color-shape configuration is the same as the SPEED ZONE target sign. Initially, the laboratory stimuli were scored with regard to the frequency of traffic signs within the cone whose color-shape configuration corresponded to that of the other three target signs. However, the variables describing these color-shapes had frequencies that were too low for meaningful data analysis. As a result, they were dropped from the regression analyses, leaving only the variables listed in Table 27. Because of variation in sign placement across target conditions within the same roadway scene, the variables listed in Table 27 required evaluation in each of the 640 slides that contained a target sign.

\section{Surround Variables}

The second class of variabies that were measured in the visual evaluation procedure were surround variables. Surround variables describe those characteristics of each roadway scene that are immedately adjacent to the target sign. As a result, the values of surround variables vary with target placement even among the nine slides of the same roadway scene. For this reason, each of the 640 sidides containing target signs was evaluated along the dimensions of all of the surround variables. Two groups of surround variables were rated. These were (1) variables describing visual features within a radius of $1^{\circ}$ or $2^{\circ}$ of yisual angle surrounding the target center and (2) variables describing the outside perimeter of the target sign. 
Table 26. Scene Variables Measured within the Four Zones of a Roadway scene $(\mathrm{N}=80)$

\begin{tabular}{|c|c|c|}
\hline $\begin{array}{c}\text { SCENE } \\
\text { VARIABLES }\end{array}$ & $\begin{array}{c}\text { ACTUAL } \\
\text { VARIABLE VALUES }\end{array}$ & $\begin{array}{l}\text { CODED } \\
\text { VARIABLE VALUES }\end{array}$ \\
\hline 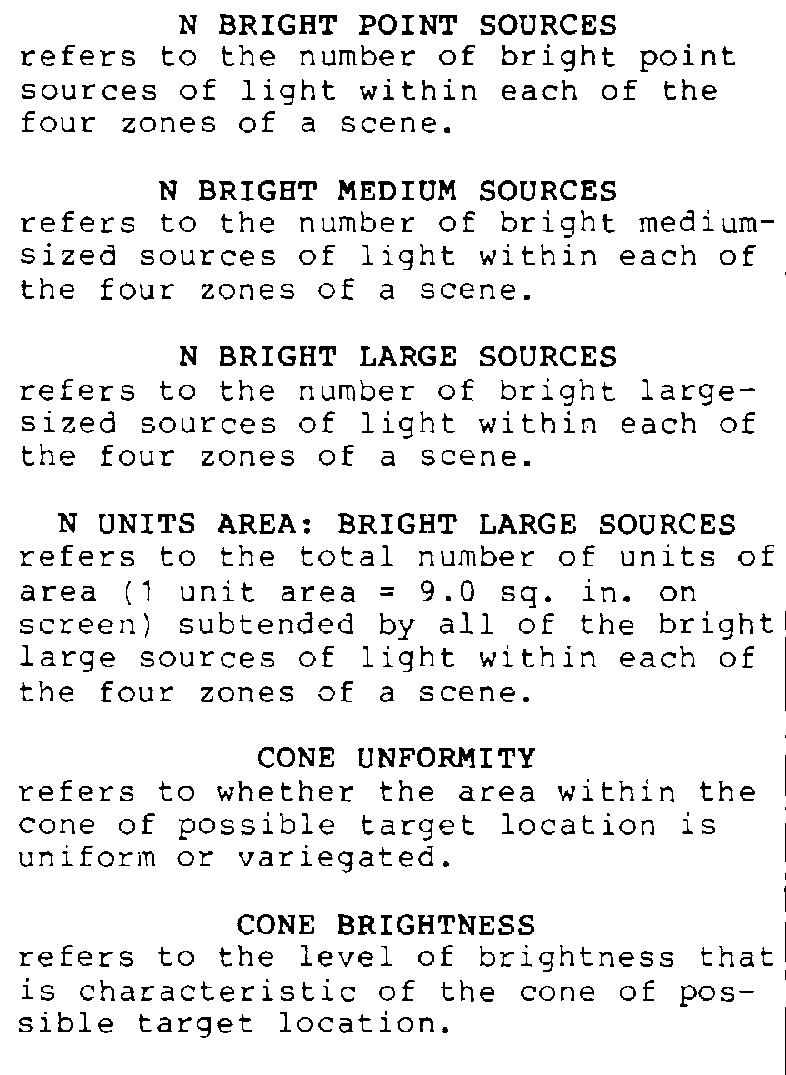 & $\begin{array}{c}>75 \% \text { uniform } \\
>25 \% \text { variegated } \\
\text { dark } \\
\text { sight } \\
\text { moderate } \\
\text { intense }\end{array}$ & $\begin{array}{l}\text { actual number: } 0-8 \\
9 \text { or more: } 9 \\
\text { actual number: } 0-8 \\
9 \text { or more: } 9\end{array}$ \\
\hline
\end{tabular}

Table 27. Scene Variables within the Cone of Possible Target Location $(\mathrm{N}=640)$

\begin{tabular}{|c|c|c|}
\hline $\begin{array}{c}\text { SCENE } \\
\text { VARIABLES }\end{array}$ & $\begin{array}{c}\text { ACTUAL } \\
\text { VARIABLE VALUES }\end{array}$ & $\begin{array}{l}\text { CODED } \\
\text { VARIABLE VALUES }\end{array}$ \\
\hline $\begin{array}{l}\text { N TRAFFIC SIGNS } \\
\text { refers to the total number of } \\
\text { traffic signs within the cone } \\
\text { N TRAFFIC SIGNS: WHITE VERTICAL } \\
\text { refers to the number of traffic signs } \\
\text { whose background color-shape configur- } \\
\text { ation is a white-vertical rectangle }\end{array}$ & Erequency count & $\begin{array}{l}\text { actual number: } 0-8 \\
9 \text { or more: } 9 \\
\text { actual number: } 0-8 \\
9 \text { or more: } 9\end{array}$ \\
\hline
\end{tabular}


The first group of surround variables were those surrounding the target center within a radius of $1^{\circ}$ or $2^{\circ}$ of visual angle. On the projection screen in the experimental room, $1^{\circ}$ of visual angle subtended about 2.5 inches, and $2^{\circ}$ subtended about 5 inches. Each of the light sources counted within the $1^{\circ}$ radius of the target center was also counted within the $2^{\circ}$ radius. Table 28 summarizes the candidate surround variables that were evaluated within both a $1^{\circ}$ radius and a $2^{\circ}$ radius of the center of the target sign. In determining the number of various-sized sources of bright light within these areas, the criteria that were applied to define "point," "medium," "large," and "bright" were the same as those utilized for the scene variables. As was true of the scene variables within the cone of possible target location, variables relevant to the number of traffic signs with the same color-shape as the STOP, DETOUR, and Pedestrian Crossing targets were dropped from the regression analyses because of insufficient frequencies. This left only the variable corresponding to the SPEED zONE target, i.e. $N$ TRAFFIC SIGNS: WHITE VERTICAL

RECTANGLES.

Table 28. Surround Variables within $1^{\circ}$ and $2^{\circ}$ Radius of Target Center $(\mathrm{N}=640)$

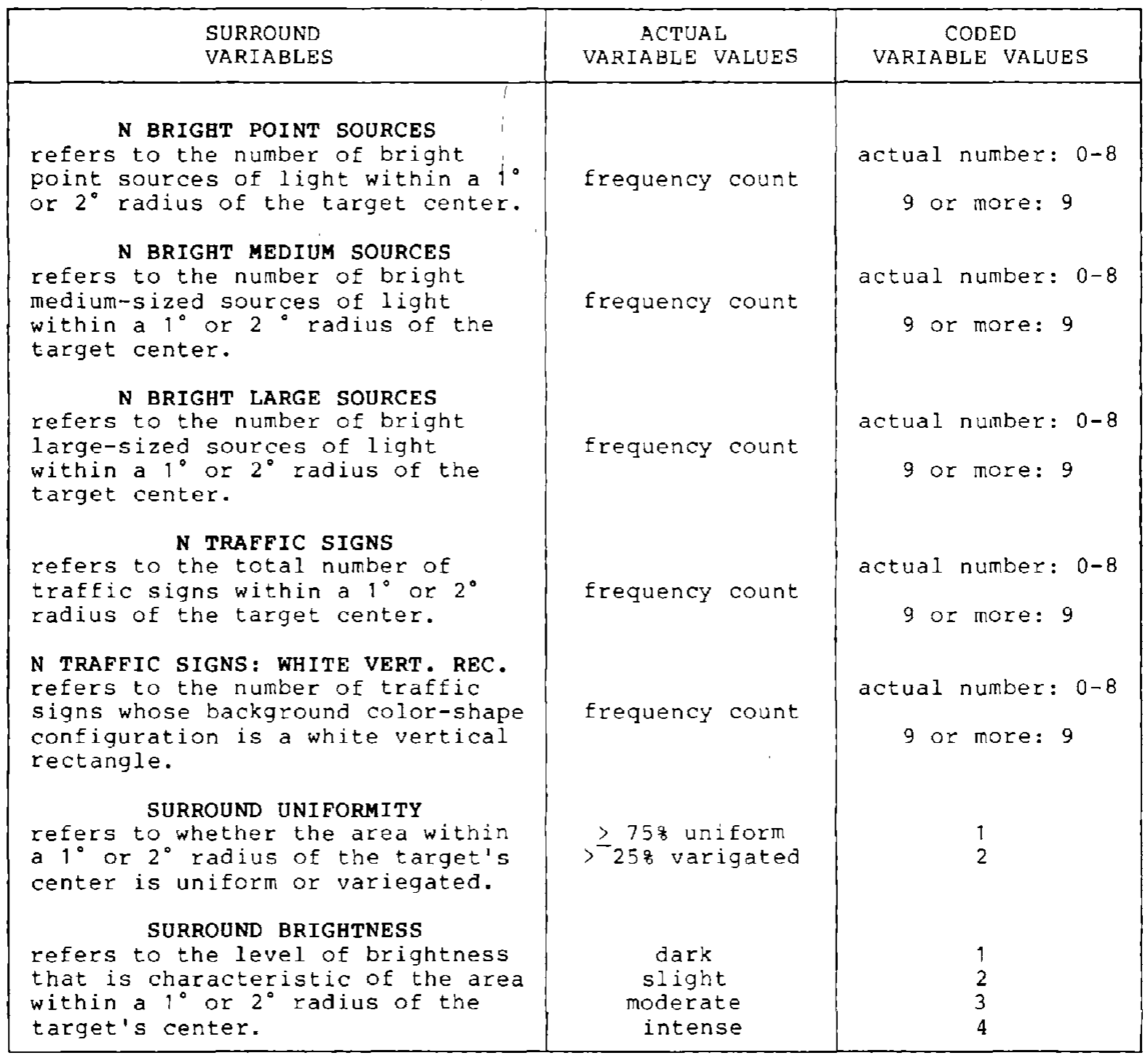


The second qroup of surround variables, those relevant to visual characteristics situated along the outside perimeter of the target sign, are listed in Table 29. The outside perimeter of the target sign has been defined as the line of scene elements contiguous to each edge of the target sign.

\section{Contrast Variables}

Contrast variables comprise the third class of variables assessed in the evaluation procedure. Contrast variables describe the difference in brightness between the target sign and those elements of the road scene that are contiguous to the sign. Since relative brightness differences are a function of both sign brightness and location, this class of variables was evaluated on each of the 640 laboratory stimuli that contained targets. The contrast variables are listed in Table 30 . The three variables listed have been scaled so that the entire sign perimeter is divided into three exclusive categories. For a given slide, then, the actual values of these three variables are additive to

100 percent.

Table 29. Surround Variables Relevant to the Target's Outside Perimeter $(\mathrm{N}=640)$

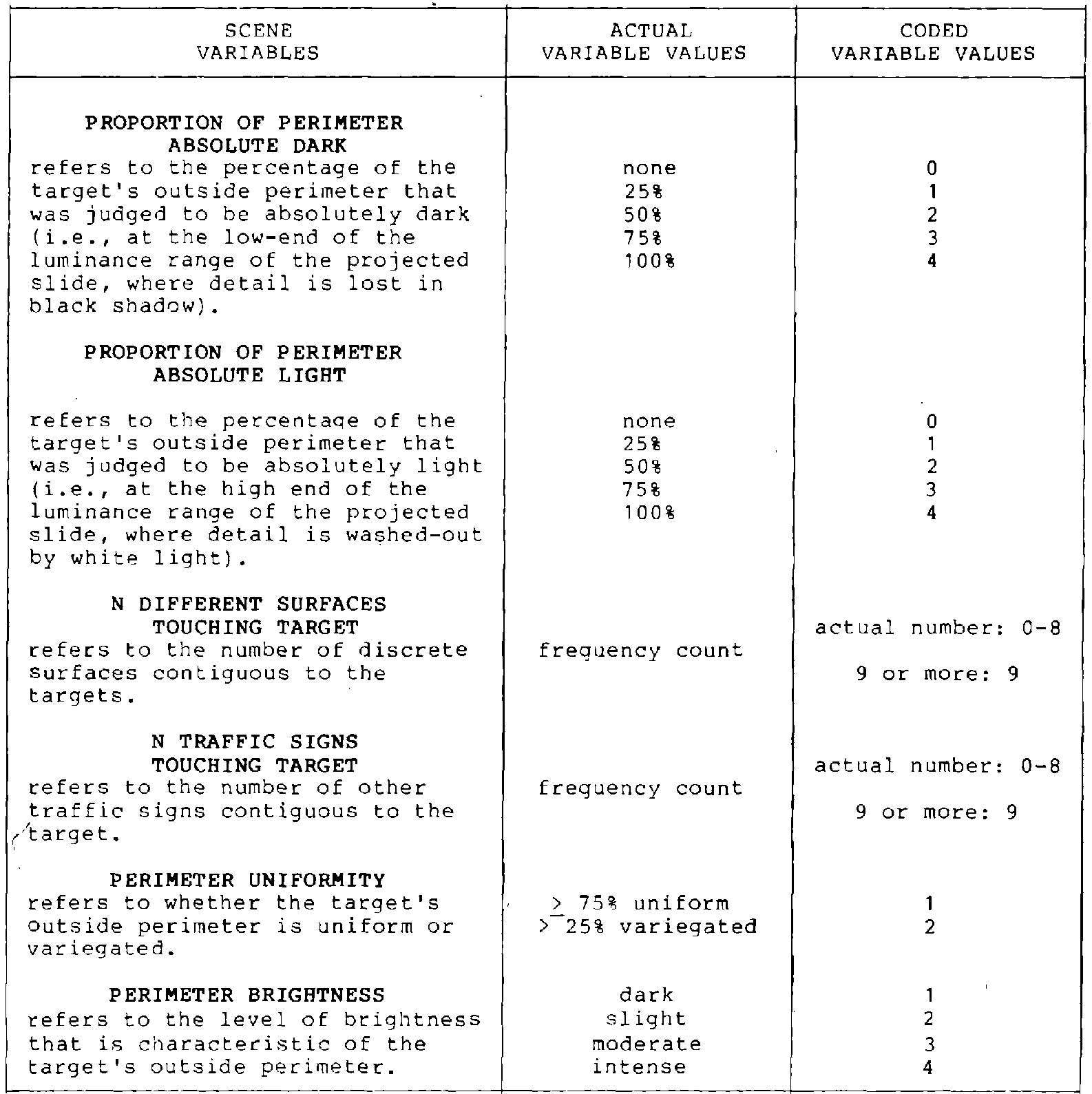


Table 30. Contrast Variables

$(\mathrm{N}=640)$

\begin{tabular}{|c|c|c|}
\hline $\begin{array}{c}\text { SCENE } \\
\text { VARIABLES }\end{array}$ & $\begin{array}{c}\text { ACTUAL } \\
\text { VARIABLE VALUES }\end{array}$ & $\begin{array}{l}\text { CODED } \\
\text { VARIABLE VALUES }\end{array}$ \\
\hline $\begin{array}{l}\text { PROPORTION OF PERIMETER DARKER } \\
\text { refers to the percentage of the } \\
\text { target's outside perimeter that } \\
\text { was judged to be darker than the } \\
\text { target sign. }\end{array}$ & $\begin{array}{l}\text { none } \\
258 \\
508 \\
758 \\
1008\end{array}$ & $\begin{array}{l}0 \\
1 \\
2 \\
3 \\
4\end{array}$ \\
\hline $\begin{array}{l}\text { PROPORTION OF PERIMETER EQUAL } \\
\text { BRIGHTNESS } \\
\text { refers to the percentage of the } \\
\text { target's outside perimeter that } \\
\text { was judged to be equal to the } \\
\text { target sign in terms of } \\
\text { brightness. }\end{array}$ & $\begin{array}{l}\text { none } \\
258 \\
508 \\
75 \% \\
1008\end{array}$ & $\begin{array}{l}0 \\
1 \\
2 \\
3 \\
4\end{array}$ \\
\hline $\begin{array}{l}\text { PROPORTION OF PERIMETER LIGHTER } \\
\text { refers to the percentage of the } \\
\text { target's outside perimeter that } \\
\text { was judged to be lighter than the } \\
\text { target sign. }\end{array}$ & $\begin{array}{l}\text { none } \\
258 \\
508 \\
758 \\
1008\end{array}$ & $\begin{array}{l}0 \\
1 \\
2 \\
3 \\
4\end{array}$ \\
\hline
\end{tabular}




\section{APPENDIX B}

\section{PHOTOMETRIC DIMENSIONS OF COMPLEXITY,} CONTRAST, AND SIGN

The photometric evaluation procedure was designed to complement the visual evaluation of the laboratory stimuli. Toward this end, the photo-metric evaluation was designed to obtain information applicable to the brightness characteristics of the overall scene (scene variables), the area immediately surrounding each target sign (surround variables), and the target sign relative to the immediate surround (contrast variables). Target sign measurements were obtained as a prerequisite to the computation of contrast measures and to provide a more accurate measure of sign brightness than the dichotomous measure used for assignment of stimuli to cells in the experimental design.

\section{Scene Variables}

Scene variables refer to dimensions of the overall road scene depicted in each of the laboratory stimuli. The photometric assessment of each scene was an illuminance measure of the amount of light reflected by each of the 720 slides used in the lab study. This measure was taken with each scene projected onto the projection screen in the experimental room under the same geometric and light conditions that existed during response data collection. A Gossen Lunasix 3 Exposure Meter was used to measure, at the $S^{\prime} s$ eye, slide illuminance reflected from the projection screen. The unit of measure was foot candles, and the coded variable values were actual foot candles. The illuminance measure was obtained by positioning the exposure meter on a tripod at $s$ eye position and pointing it directly at the center of the projection screen. The area measured subtended an area of $30^{\circ}$, which is approximately equal to a circle with a diameter of 6 feet 3 inches $(187.5 \mathrm{~cm})$ at the plane of the projection screen. The screen itself was 5 feet by 7 feet $(152.4 \mathrm{~cm} \times 213.4 \mathrm{~cm})$. Consequently, light reflecting from the lateral edges as well as the four corners of the screen was not included in the illuminance measure. However, these areas tended to be consistentiy dark and homogeneous across slides, so that variation across slides in the amount of light reflected from these areas of the screen should have been minimal.

\section{Surround Variables}

Surround variables were also assessed in the photometric evaluation procedure. These variables reflect the characteristics of each roadway scene that are immediately adjacent to the target sign. Because the values of surround variables vary as a function of both the target's precise location within a scene and idiosyncracies in the photographic processing of the individual scene negatives, each of the 640 slides with target signs was evaluated along the dimensions of all of the surround variables. These variables are listed in Table 31 .

All of the photometric measures of the target s surround were luminance readings, expressed and coded as footLamberts that were obtained with a Spectra-pritchard Photometer -- Model 1980. Luminance values were obtained from all 640 slides that included one of the four targets. The luminance measures were obtained under the same physical conditions that existed in the laboratory study itself. Specifically, with the photometer located at $S$ eye position, each of the experimental slides was projected onto the projection screen in the experimental room under the same geometric and light conditions established for S response data collection. One technician operated the photometer optical head, and another recorded the luminance values displayed by the digital meter readout on the control console.

Table 31. Surcound Variables* $(\mathrm{N}=640)$

SURROUND MINIMUM
refers to the lowest luminance value measured with a 6 foot aperture
along the outside perimeter of the target sign
SURROUND MAXIMUM
refers to the highest luminance value measured with a 6 foot aperture
along the outside perimeter of the target sign
suRROUND AVERAGE
refers to the average luminance of the target sign's immediate surround
measured with a $1{ }^{\circ}$ aperture at four locations contiguous to the target:
$12,3,6$, and 9 o'clock

* All actual and coded variable values are luminance readings expressed in footLamberts. 


\section{Target Variables}

Target variables refer to the brightness characteristics of the target sign. since sign brightness varied from one scene to the next, this class of variables was measured in each of the 640 slides that contained targets. Target measurements were necessary both to ensure an appropriate brightness difference in the low versus high brightness condition of a given target within a particular scene and to provide data necessary to the computation of contrast variables. Table 32 summarizes the target variables that were assessed photometrically.

All target variable values were obtained with the photometer, which was operated in the same manner utilized for surround measures. To maintain consistency across slides in the way that these variables were measured, a measurement method was established for each factor. This measurement method entailed the use as a guide of a scaled illustration of each target at each of the four distances. Each of these scaled illustrations or maps identified the appropriate aperture size(s) and location(s) to be used in measuring a specific device at a given distance. Figures 12 and 13 illustrate the maps used to guide the photometer assessment of a DETOUR sign at 400 feet. The maps for the Integrated Target readings were designed to accurately represent the average actual luminance of the entire target sign. In developing each of these maps, the "average actual luminance of the entire target sign" was first determined by dividing the area of the sign face into small cells that were just large enough to accommodate one 2minute or 6-minute aperture of the photometer. The choice of using either a 2minute or 6-minute aperture was determined by both the overall size of the device and the cell-matrix that "fit" the device most effectively. once the sign face was divided into cells, a luminance reading was taken from each of the cells. The average sign Iuminance was then computed. Following this, various configurations of larger-sized apertures were evaluated to identify a sample of readings that most accurately represented the integrated luminance of the sign face. Once the integrated sampling map had been established in this way, it was validated by repeating the entire procedure with a second slide containing the same target at the same distance at a different level of brightness.

For the Target Legend readings, the target characteristic measured was not the same across distances even for the same given device. This is because it was usually not possible for the stroke width of literal legends to accommodate the smallest aperture available on the photometer optical head. In these cases, the aperture was fitted within the boundaries of the word's shape configuration; consequently, the reading included both discrete legend strokes and the immediately adjacent sign background among the strokes. In addition, the strokes of legends on devices at the 600 foot distance were not even discernible. Instead, the word STOP, for example, appeared as a fuzzy white horizontal line

Table 32. Target Variables $(N=640)$

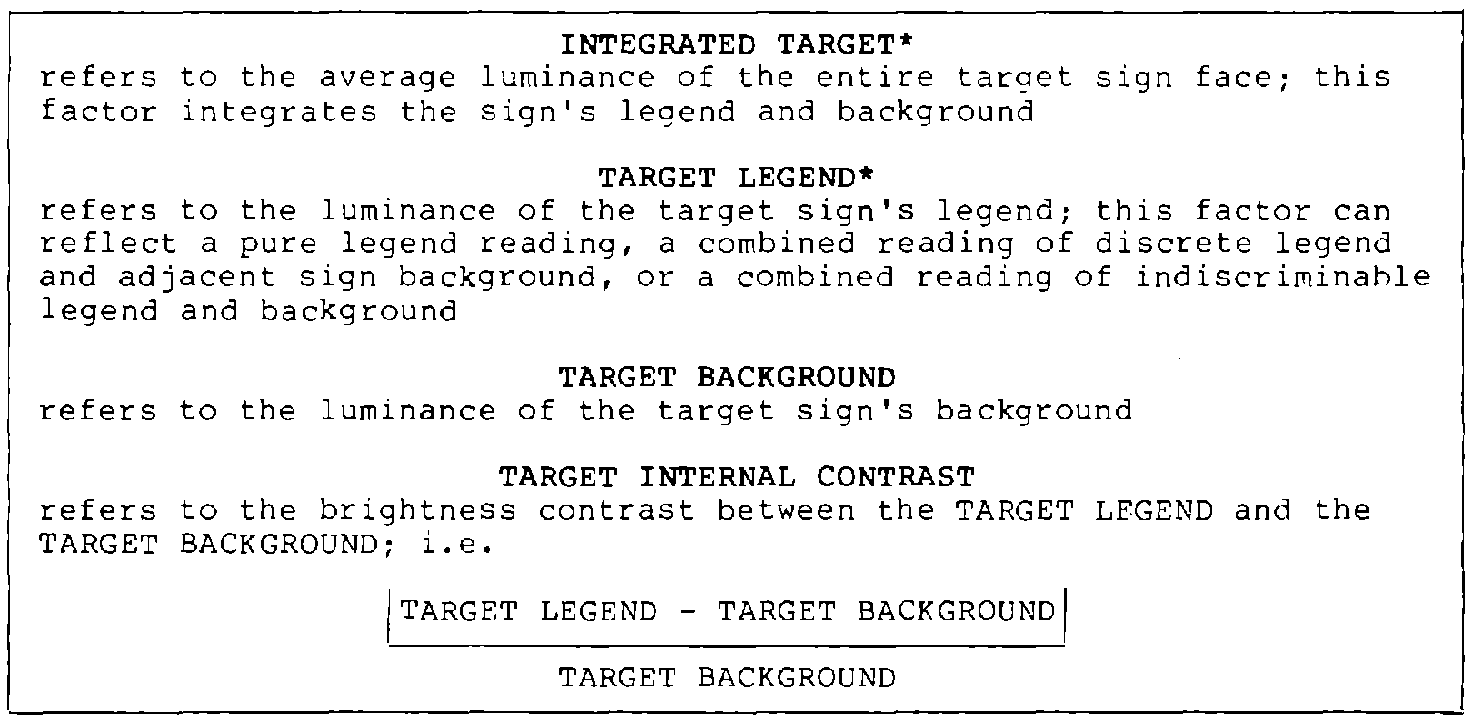

* Actual and coded variables values are luminance readings expressed in footLamberts. 


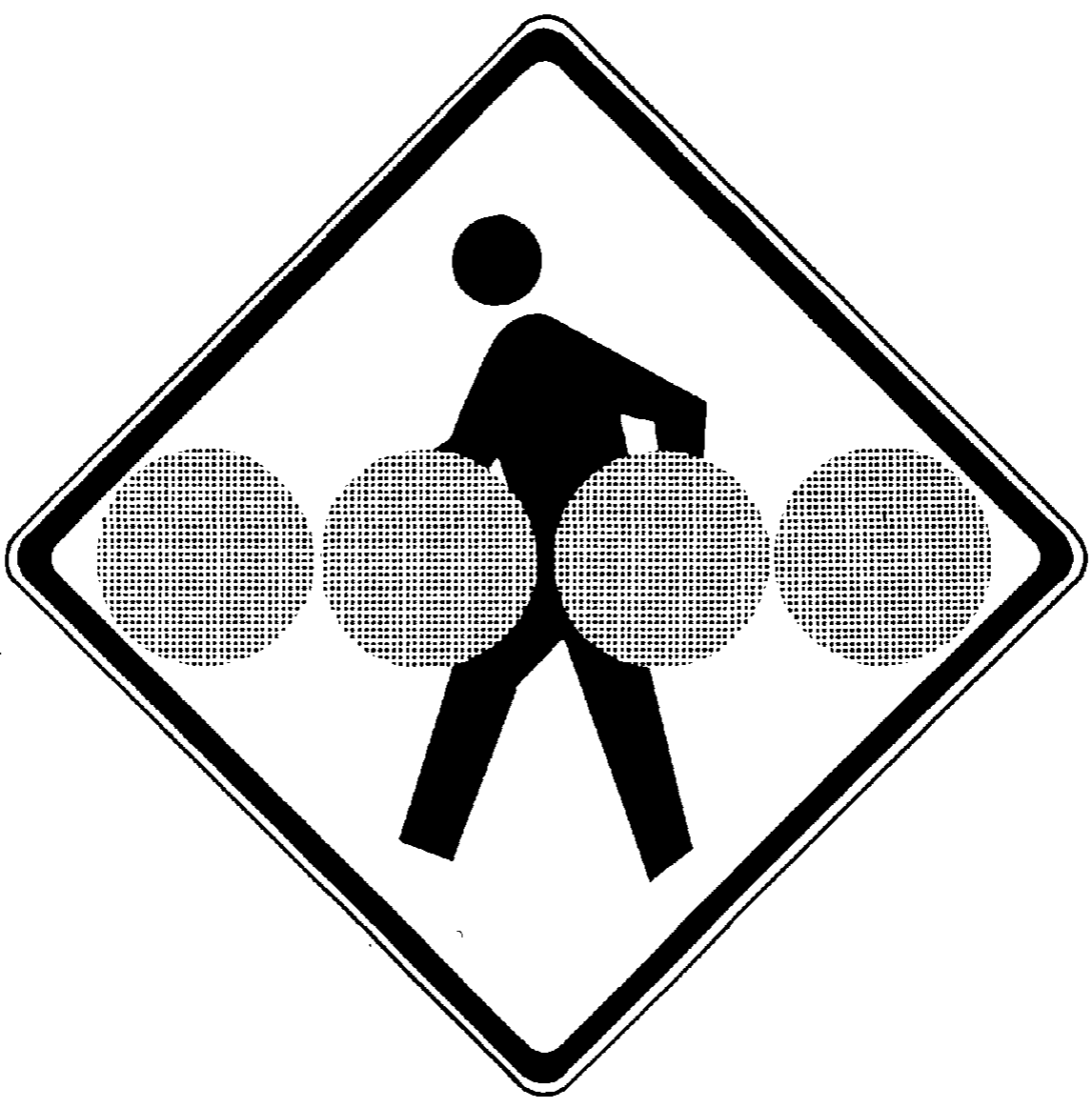

APERTURE SIZE $=6$ minutes

SCALE $=0.138=1$ inch

Figure 12. Scaled Illustration of Aperture Locations for

Target Integrated Readings of Pedestrian Crossing Sign at 400 Feet

One foot $=.305$ meters.

One inch $=2.540$ centimeters. 


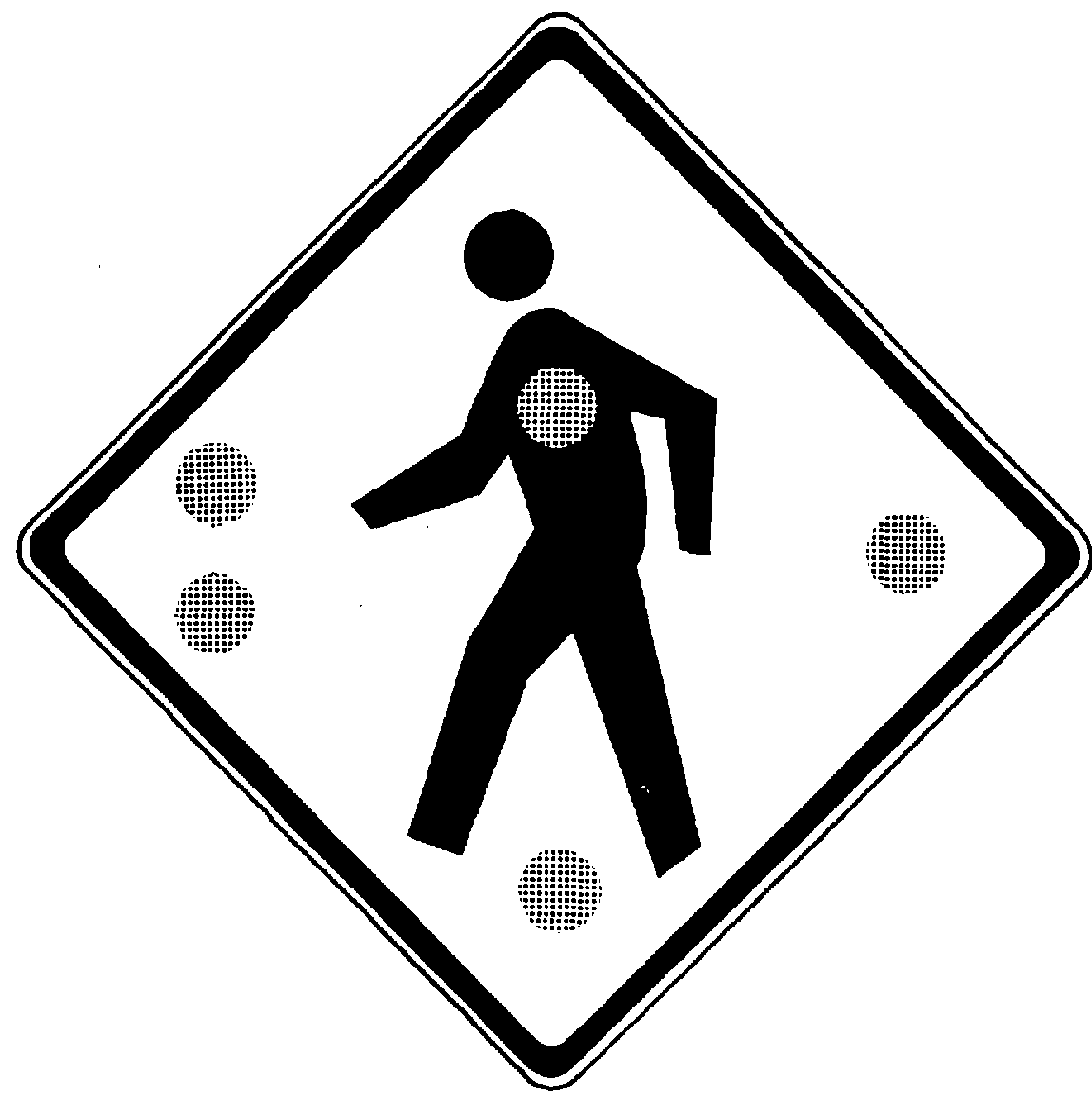

$$
\begin{aligned}
& \text { APERTURE SIZE }=2 \text { minutes } \\
& \text { SCALE }=0.138=1 \text { inch }
\end{aligned}
$$

Figure 13. Scaled Illustration of Aperture Locations for Target Legend and Target Background Readings for pedestrian Crossing Sign at 400 Feet

One foot $=.305$ meters.

One inch $=2.540$ centimeters. 
across the face of the sign. Likewise, the words DETOUR and SPEED ZONE AHEAD appeared as fuzzy light gray horizontal lines. When this was true, the aperture was fitted within the confines of the fuzzy white/gray line. Finally, at the 800 foot $(240 \mathrm{~m})$ distance, legends were generally not discriminable from the sign backgrounds at all. As a result, neither legend nor background readings were taken from targets at the 800 foot $(240 \mathrm{~m})$ distance. Table 33 identifies the physical Target Characteristic reflected by the Target Legend variable for each target at all four distances.

The last target variable listed in Table 32 is Target Internal contrast. The value of the variable was computed from the Target Legend and Target Background readings: it reflects the brightness contrast between these two variables expressed as a positive ratio. As such, this variable did not require any additional photometric measures.

\section{Contrast}

The final class of photometric variables was a series of brightness contrast ratios between the target and its immediate surround. These ratios were derived from the target and surround luminance readings and did not require any other measures. The photometric contrast variables used in the regression analyses are 1 isted in Table 34 . The values of these variables were computed for each of the 640 stimuli containing target signs.

Initially, an additional, similar series of external contrast measures was also calculated. These contrast measures were th same as those 1 isted in Table 34 except that Integrated Target brightness was substituted for Target Background brightness in the contrast ratio equations. However, the intercorrelations between corresponding contrast measures proved to be extraordinarily high. Because of this high colinearity and because eliminating one of the two types of contrast ratios did not noticeably alter the magnitude of the Multiple Rs in regression analyses, it was decided to select only one series of contras ratios (i.e. those derived from Target Background measures versus those derived fro Integrated Target measures). Both types of contrast ratio appeared to correlate equally well with subject performance, thereby eliminating subject performance as a criterion for selection. Ultimately, the contrast ratios based on pure Target Background readings were selected for use in analysis because the pure background measure could be more reliably obtained than the integrated readings and because pure background readings have been traditionally used to compute external contrast.

Table 33. Physical Target Characteristic Measured for Target Legend Variable

\begin{tabular}{|c|c|c|c|c|c|}
\cline { 2 - 6 } \multicolumn{1}{c|}{} & \multicolumn{5}{c|}{ DEVICE } \\
\hline $\begin{array}{c}\text { TARGET } \\
\text { DISTANCE }\end{array}$ & $\begin{array}{c}\text { Detour } \\
\text { arrow }\end{array}$ & "DETOUR" & $\begin{array}{c}\text { Pedestrian } \\
\text { Crosssing }\end{array}$ & STOP & $\begin{array}{c}\text { SPEED } \\
\text { ZONE }\end{array}$ \\
\hline 250 & 1 & 4 & 1 & 1 & 2 \\
400 & 1 & 2 & 1 & 2 & 2 \\
600 & 1 & 3 & 1 & 3 & 3 \\
800 & 4 & 4 & 4 & 4 & 4 \\
\hline \multicolumn{7}{|c|}{ REY } \\
\hline 1 & completely within letter stoke boundaries \\
\hline 2 & word configuration: includes discrete legend \\
\hline 3 & strokes and sign background \\
\hline 4 & fuzzy white/gray line formed by legend \\
\hline
\end{tabular}


Table 34. External Contrast* $(\mathrm{N}=640)$

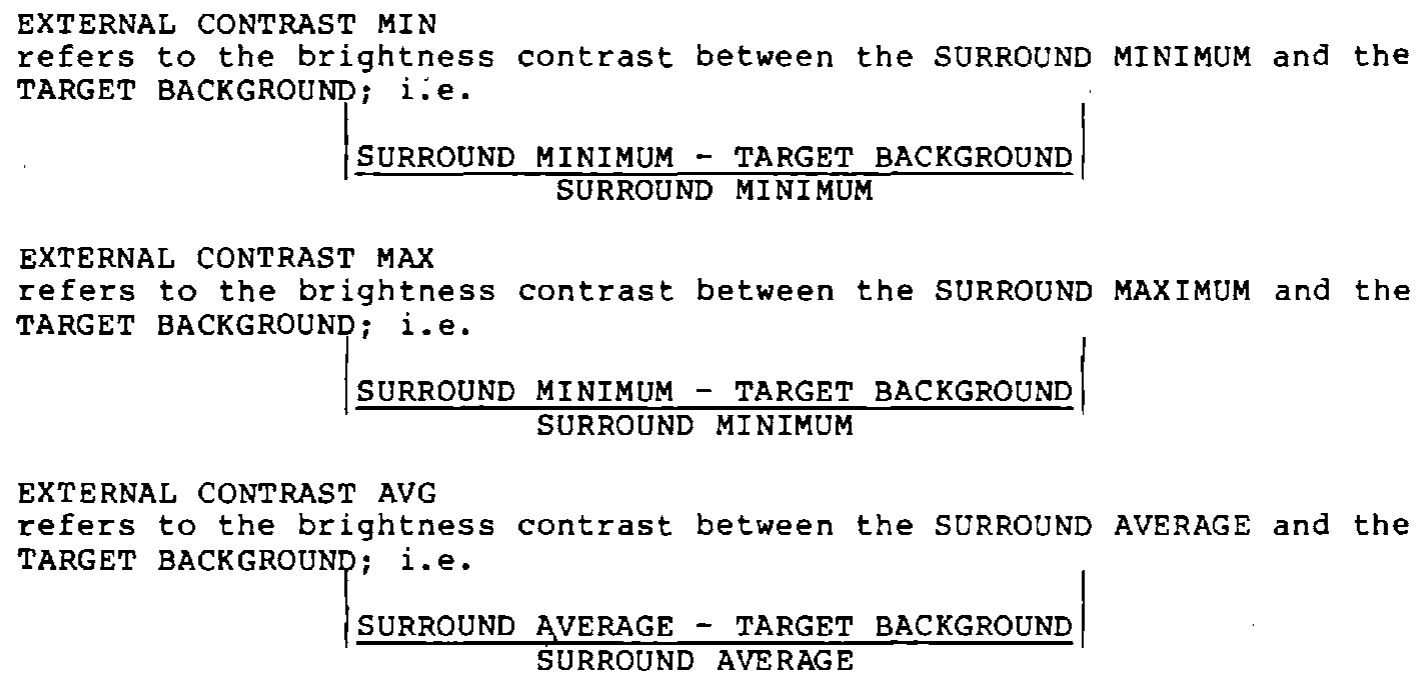

* All contrast ratios were computed from luminance readings expressed in footLamberts. 


\section{APPENDIX C}

\section{EXPERIMENT INSTRUCTIONS}

\section{Session I}

The project in which you are participating is a study of driver vision. The purpose of this study is to determine how well drivers can see certain objects in different nighttime driving situations. As you probably already know, being a subject in this experiment requires that you attend a total of three experimental sessions that last about two hours each. This is the first of these three sessions. During each session you will see different nighttime road scenes projected onto the screen in front of you, and you will be asked to look for certain objects in each of these scenes. After you have attended all three sessions, you will be paid for participating in the study. The amount that you will be paid depends on how well you perform in the experiment: this amount can vary from a minimum of $\$ 15.00$ to a maximum of $\$ 24.00$.

Before we begin, you must first become familiar with what you are to do. As I mentioned before, you will be asked to look for certain objects in a series of projected nighttime road scenes. ¿ach of the projected scenes is similar to what a driver sees as he looks at the road ahead. Here are two examples of a nighttime road scene.* The objects that you will be looking for as you view these scenes are the same things that drivers must search for in order to avoid accidents. That is, a safe driver pays attention to the road on which he is travelling, the location of other traffic around him, and traffic signs. Similarly, for each of the scenes you are shown, you should pay attention to the road, to other traffic, and to traffic signs.* These three categories - Road, Traffic, and Signs -- are listed in the first column of the table being projected now. The middle column of this table lists the things within each category that you will be required to search for. The last column of this table lists the labels you will use to identify each of these things. Let's consider more closely the things to look for within each of the three categories.

In the category labelled "Road," there are two things to look for. The first is the presence of a curve in the road on which you are travelling. The label used to indicate the presence of a curve in the road ahead is "curve." The second thing in the "Road" category is the pres- ence of any painted $I$ ines between the edges of the road. This can be represented by any type of center line, lane line, or edge line, white or yellow. The labels for these lines are "solid" when there is a solid line and "dashed" when there are dashed 1 ines. These labels are words you will use to tell us what you see in a scene. For example, in looking at a scene, if you see the road is curved, say "curve"; if you see a solid center line and dashed lane line, say "solid" and "dashed."

The category labelled "Traffic" includes two things. The first is the presence of any other vehicles moving in the same direction that you are travelling. The label associated with this is "same." The second thing consists of any vehicles moving in the opposite direction. The label for this is "opposite."

The "Sign" category contains four things. The first is a stop sign, and its label is "stop." The second thing is a sign that says "speed zone ahead," and its label is "speed zone." The next is a sign that says "detour," and the label for this sign is "detour." The fourth thing in the "Sign" category is a pedestrian crossing sign, and its label is "crossing."

Now that you know the nine things to look for and their labels, you will see how these things appear in the projected road scenes.

The category labelled "Road" includes two things: a curve in the road ahead and the presence of either solid or dashed lines. A curve in the road ahead may be either sharp or gentle; the curve may be either to the right or the left. If the road ahead curves in any of these ways, then the label "curve" should be used to indicate this. The curve shown here is a gentle curve to the left. * The second and third things in the "Road" category consist of any solid or dashed painted lines between the edges of the road; this includes any type of center line, lane line, or edge line. In this example* we see a dashed lane 1 ine and your response would be "dashed." In this example* we see a double solid center 1 ine and your response should be "solid." In this example* we see a dashed lane line and a solid edge 1 ine and your response should be "solid and dashed."

There are two things in the "Traffic" category: traffic moving in the same direction, and traffic moving in the opposite direction. "Traffic" refers to

* Example slide(s) shown. 
any other motorized vehicle: it can be a car, a truck, or a motorcycle. The traffic may be either near or far. In the example, we see traffic in both directions and your response should be "same and opposite. The label for traffic moving in the same direction is "same,". for traffic moving in the opposite direction, the label is "opposite."

The category labelled "sign" contains four things: a stop sign, whose label is "stop"; a speed zone ahead sign whose label is "speed zone"; a detour sign, whose label is "detour" ; and a pedestrian crossing sign, whose label is "crossing." These signs can either be near or far.

The next series of slides illustrates how these four signs appear. when they are near. * At the far distances, the signs are smaller and cannot be read. The next four slides show how the signs look when they are far away. $*$

Notice that at the far distance, the stop sign appears as a dark red circle with a white rectangle across it. t This is how a speed zone ahead sign looks from far away. It is a white rectangle with three horizontal light gray lines. * This slide shows how the detour sign appears from the far distance. It is an orange rectangle that has a light gray horizontal line across the top and a darker arrow. accoss the bottom.* This is the pedestrian crossing sign from the far distance. Notice that it is a yellow diamond that has a short black vertical mark on it. For all four. signs at the far distance, the markings on the sign. faces may be very difficult to see in some scenes.

There will always be one, but never be more than one, of these four signs located in each projected road scene, and it will always be located on the right side of the road. of course, there may be other traffic signs in the scenes in addition to these four signs; but you should only answer when you have seen one of the four signs we have discussed -ignore any other signs. If you are not certain. which of the four signs. is in a scene, please do the best you can and make your best guess. Doing this can only increase the amount you are paid.

Your task" is to look for these nine things (PROJECTED LABELS)* in the projected road scenes and call out the labels of the things that you see. In order to be able to do this, you must memorize the list of labels so that you can search for these nine things quickly and accurately. Before continuing, then, please memorize the list of labels being projected now. When you feel that you are able to recite this list without looking at it, let me know.

Now that you know the labels, we must be sure that you can accurately use the label to describe things in roadway scenes. * Please look at some scenes as I point out the things to be found in each and call out their labels (PRACTICE) * Now for the next series of scenes, .I will point out the things, and you should call out the appropriate labels "(PRACTICE) * "For the following series of scenes, you" should look for the nine things and call out their labels by yourseif - without me pointing at anything (PRACTICE).*

We are now ready for some practice that is similar to the actual study: Up until now, you have seen each scene for a relatively long period. of time.. In this. practice, each scene will be shown for the same brief duration that is used in the study itself. Each slide will be preceded by a buzzer, which is a signal that the scene will be projected in one second. Just after the buzzer, the scene will be shown for 3 seconds. During this time, you should look for the nine things in the Road, Traffic and Sign categories and call out the appropriate labels.

There is no need to complete your answer while the scene is being projected. You may call out the labels while the scene. is being projected, or immediately after viewing the scene, or both., During this part of the,practice, we will discuss your answers between scenes. Do you have any questions? Please look at the screen in front of you and wait for-the "Ready Buzzer".: (PRACTICE WITH FEEDBACK). *

We are now ready- for the last part of practice trials, which is exactly the same as the study itself. For this practice the scenes will be projected just as before except that there will be a 15second interval between scenes. We will not discuss your answers for these practice trials. . Do you have any questions? Please look at the screen and wait for the "Ready Buzzer". (PRACTICE) .*

We will take a 1-minute break now before we begin the first 20-minute session, after which you will have another break (BREAR TIME).

Before we begin, please review the list of nine target labels once again (PROJECTED LABELS).* I want to emphasize that your performance and therefore what

t Example slide(s) shown. 
you will be paid will depend on your proper response to all nine things in the Road, Traffic, and Sign Categories. Do not try to get some of the items correct and ignore others.

Do you have any questions? Let's begin. The first scene will be projected soon.

\section{EXPERIMENT INSTRUCTIONS}

Sessions II \& III

As you probably remember, your task in this study is to look for nine different things in each nighttime road scene that is projected onto the screen in front of you. Searching for these nine things requires that you pay attention to the road, other traffic, and traffic signs.* These three categories -- Road, Traffic, and signs -- are listed in the first column of the table being projected now. The midale column of this table lists the things within each category that you are to search for. The last column of the table lists the labels you are to use to identify each of these things. Let's consider more closely the things to look for within each of the three categories.

In the category labelled "Road," there are two things to look for. The first is the presence of a curve in the road on which you are travelling. The label used to indicate the presence of a curve in the road ahead is "curve".* A curve in the road ahead may be either gentle or sharp - to the right or to the left. The curve in this scene is a gentle curve to the left. The second thing to look for in the "Road" category is the presence of any painted lines between the edges of the road -- including any type of center line, lane line, or edge line - white or yellow. The labels for the lines are "solid" when there is a solid line and "dashed" when there are dashed lines.* In this scene, we see a dashed lane line and a solid edge line, and your response should be "solid and dashed."

The category labelled "Traffic" includes two things. The first is the presence of any motorized vehicle moving in the same direction that you are travelling. The label associated with this is "same." The second thing consists of any vehicles moving in the opposite direction. The label for this is "opposite."* In this example, there is traffic in both directions, and your response should be "same and opposite."
The category labelled "Sign" contains four things: a stop sign, whose label is "stop," a speed zone ahead sign, whose label is "speed zone," a detour sign, whose label is "detour," and a pedestrian crossing sign, whose label is "crossing." These signs can be either near or far.

The next series of slides illustrates how these four signs appear when they are near.* At the far distances, the signs are smaller and cannot be read. The next four slides show how the signs look when they are far away. * Notice that at the far distance, the stop sign appears as a dark red circle with a white rectangle across it.* This is how a speed zone ahead sign looks from far away. It is a white rectangle with three horizontal light gray lines." This slide shows how the detour sign appears from the far distance. It is an orange rectangle that has a light gray horizontal line across the top and a darker arrow across the bottom.* This is the pedestrian crossing sign from the far distance. Notice that it is a yellow diamond that has a short black vertical mark on it.* For all four signs at the far distance, the markings on the sign faces may be very difficult to see in some scenes.

There will always be one, and only one, of these four signs in each scene, and it will always be located on the right side of the road. If you are not certain which of the four signs is in a scene, you should make the best guess that you can. Doing this will increase the amount that you are paid.

Your task is to look for these nine things (PROJECTED LABELS) * in the projected road scenes and call out the labels of the things that you see. In order to be able to do this, you must memorize the list of labels so that you can search for these nine things quickly and accurately. Before continuing, then, please memorize the list of labels being projected now. When you feel that you are able to recite this list without looking at it, let me know.

We are now ready for some practice, which is exactly the same as the study itself. For this practice, the scenes will be projected for a period of 3 seconds each, and there will be a 15-second interval between scenes. Do you have any questions? Please look at the screen and wait for the "Ready Buzzer" (PRACTICE).*

We will take a 1-minute break now before we begin the first 20-minute session,

*Example slide(s) shown. 
after which you will have another break (BREAK T TME):

Before we begin, please review the list of nine target labels once: again (PROJECTED LABELS). * I want to emphasize that your performance and therefore what you will be paid will depend on your proper.response to all nine things in the Road, Traffic, and Sign Categories. Do' not try to get some of the items correct and ignore others.

Do you have any questions?. Let's begin. The first scene will be projected soon.

* Example slide(s) shown. 


\begin{tabular}{|c|c|c|c|c|c|c|c|}
\hline \multirow{4}{*}{ 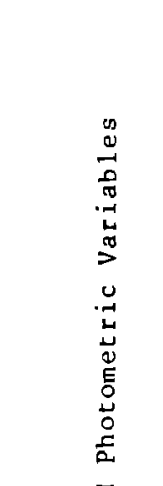 } & \multirow{3}{*}{ 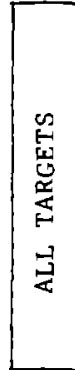 } & \begin{tabular}{|c|}
$\vec{\infty}$ \\
0 \\
0 \\
11 \\
$a$ \\
\end{tabular} & ـ & 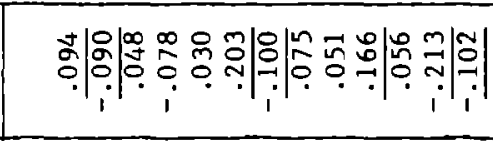 & 吕 & \begin{tabular}{llll}
$\vec{n}$ & 0 & 0 & 0 \\
0 & 8 & 0 & 0 \\
\hdashline & 0 & 0 \\
1 & 0
\end{tabular} & 象 \\
\hline & & \multirow{2}{*}{$\begin{array}{l}0 \\
9 \\
0 \\
11 \\
z\end{array}$} & $\infty$ & 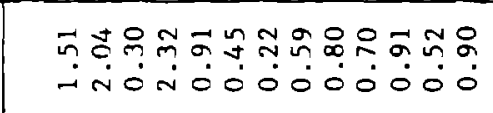 & 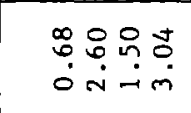 & 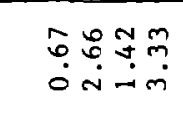 & 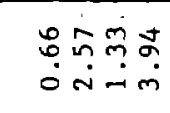 \\
\hline & & & $\mid x$ & 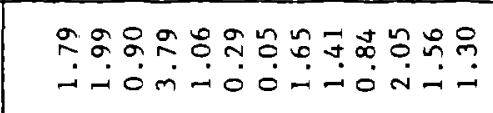 & 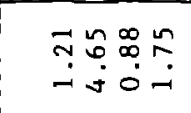 & 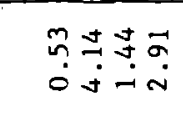 & 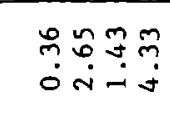 \\
\hline & $\underline{z}$ & $\stackrel{n}{n}$ & $\omega$ & 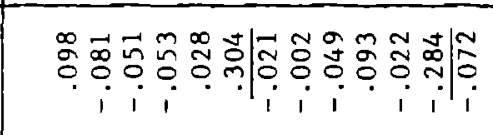 & 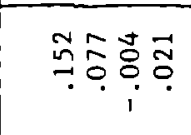 & 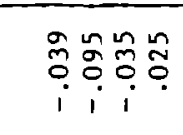 & oิ స్ำ \\
\hline$\overbrace{0}^{\pi}$ & 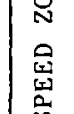 & 8 & $\infty$ & 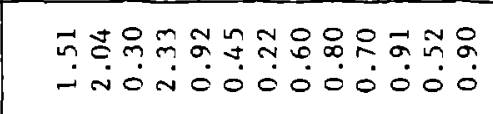 & 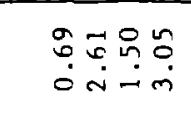 & 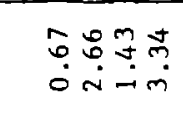 & 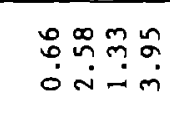 \\
\hline $\begin{array}{l}3 n \\
=0 \\
\bar{x}\end{array}$ & & $z$ & $\mid x$ & 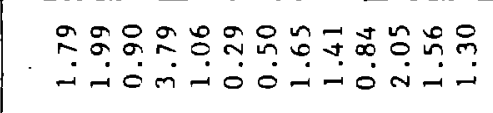 & 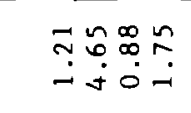 & 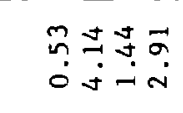 & 雚: \\
\hline 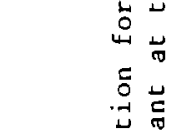 & & $\stackrel{n}{n}$ & - & 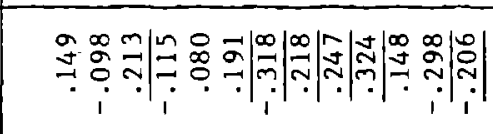 & 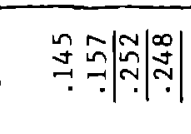 & 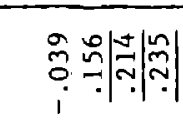 & 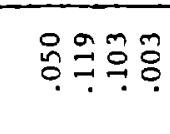 \\
\hline 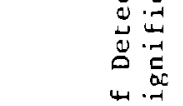 & 总 & $\stackrel{8}{0}$ & $\infty$ & 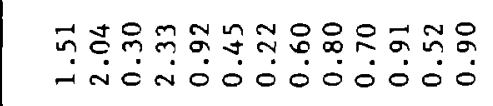 & 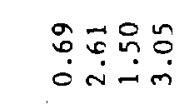 & bْ: & 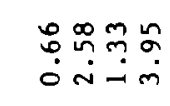 \\
\hline 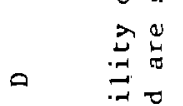 & & $z$ & $\mid x$ & 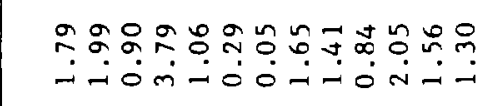 & 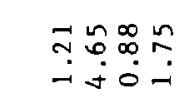 & 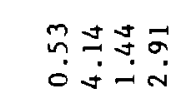 & 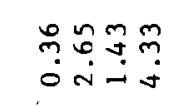 \\
\hline 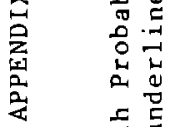 & $z_{-}^{U}$ & $\stackrel{n}{n}$ & - & 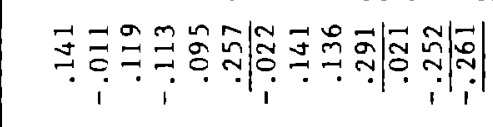 & 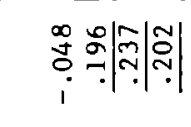 & 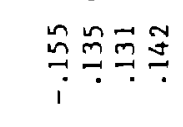 & 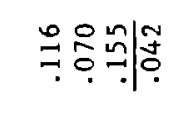 \\
\hline 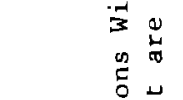 & $\begin{array}{l}\text { Do } \\
\text { : } \\
0\end{array}$ & $\stackrel{8}{:}$ & $\infty$ & 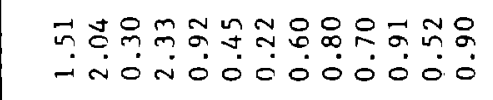 & 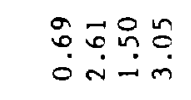 & 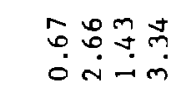 & 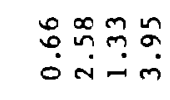 \\
\hline 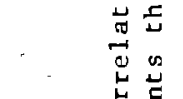 & $\frac{[x]}{a x}$ & $z$ & $\mid x$ & 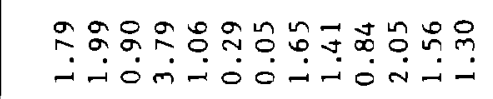 & 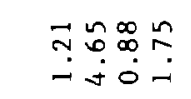 & 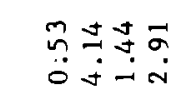 & m̊n \\
\hline 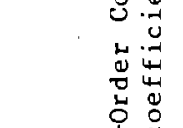 & & $\stackrel{n}{n}$ & $\mu$ & 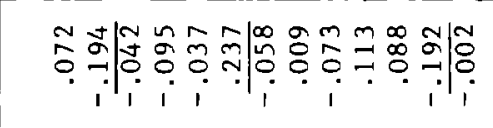 & $\exists ㅋ$ & 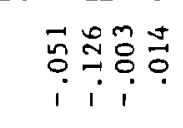 & 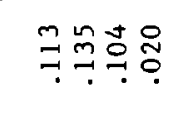 \\
\hline 莣. & 产 & $:$ & in & 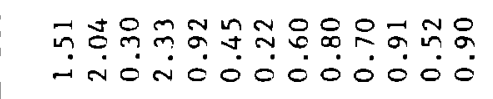 & 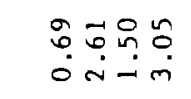 & 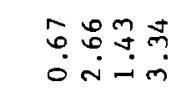 & 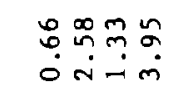 \\
\hline 至 & & z & $\mid x$ & 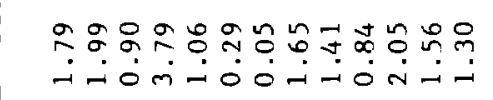 & 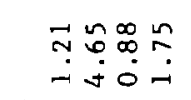 & 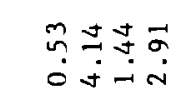 & 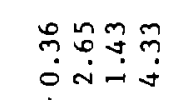 \\
\hline 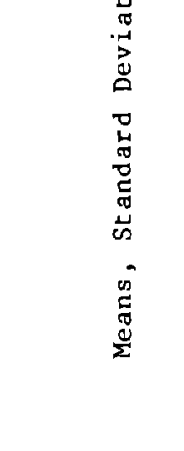 & & 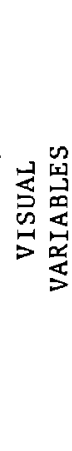 & & 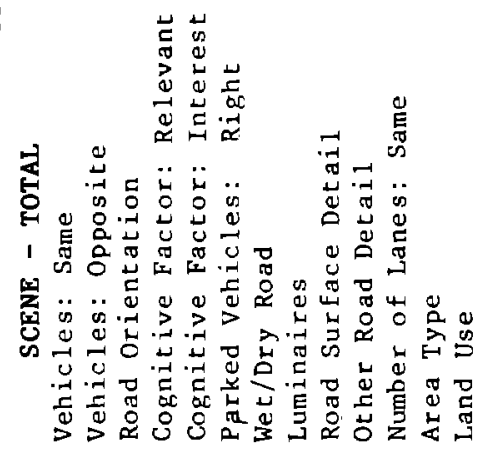 & 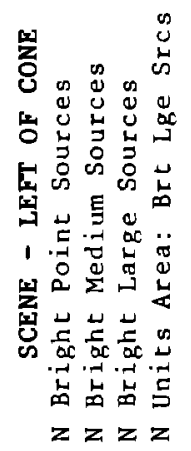 & 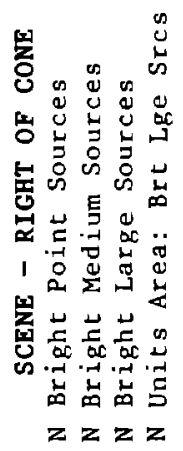 & $\begin{array}{l}r \\
\end{array}$ \\
\hline
\end{tabular}




\begin{tabular}{|c|c|c|c|c|c|}
\hline \multirow{3}{*}{ 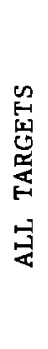 } & $\begin{array}{l}-1 \\
\infty \\
0 \\
11 \\
0\end{array}$ & $H$ & 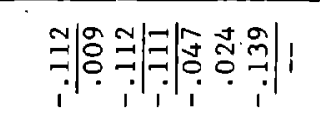 & 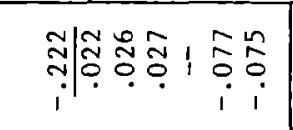 & 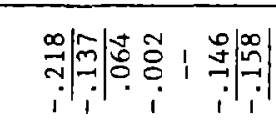 \\
\hline & \multirow{2}{*}{\begin{tabular}{l}
0 \\
\multirow{J}{0}{} \\
11 \\
$z$
\end{tabular}} & $\infty$ & 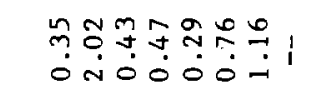 & ํㅠㅁㄴ: & 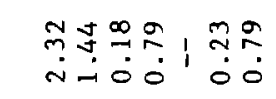 \\
\hline & & $\mid x$ & 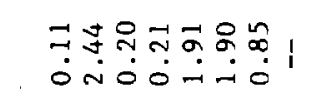 & 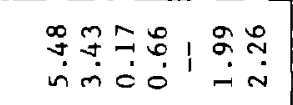 & 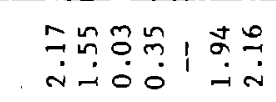 \\
\hline \multirow{3}{*}{ 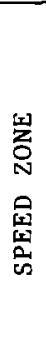 } & \begin{tabular}{l}
$n$ \\
$n$ \\
\hdashline \\
$a$
\end{tabular} & $H$ & 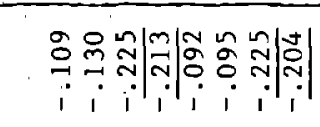 & 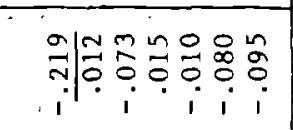 & 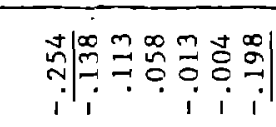 \\
\hline & \multirow{2}{*}{$\begin{array}{l}0 \\
8 \\
0 \\
11 \\
z\end{array}$} & $\infty$ & 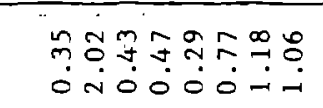 & 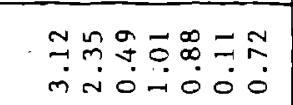 & 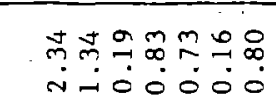 \\
\hline & & $\mid x$ & 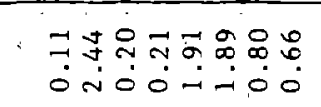 & 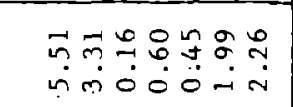 & 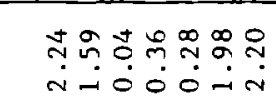 \\
\hline \multirow{3}{*}{ 号 } & $\begin{array}{l}n \\
i n \\
i \\
a\end{array}$ & מ. & 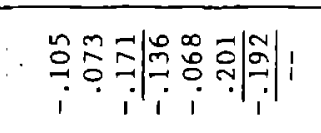 & 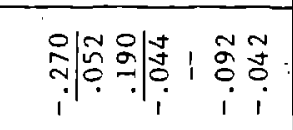 & 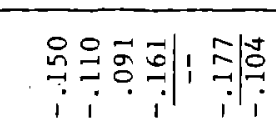 \\
\hline & \multirow{2}{*}{ 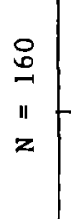 } & $\infty$ & 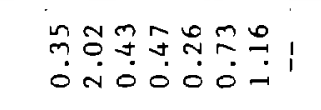 & 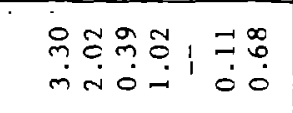 & 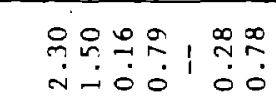 \\
\hline & & $|x|$ & 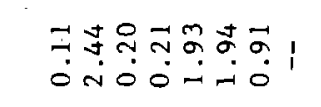 & 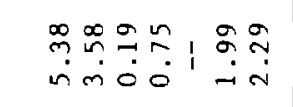 & 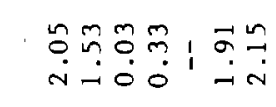 \\
\hline \multirow{3}{*}{$\begin{array}{l}0 \\
Z \\
0 \\
0 \\
0 \\
0 \\
0 \\
0 \\
\dot{0} \\
\dot{0}\end{array}$} & in & $t$ & 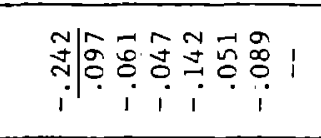 & 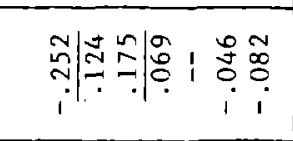 & 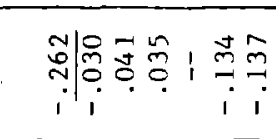 \\
\hline & \multirow{2}{*}{\begin{tabular}{l}
8 \\
\hdashline \\
11 \\
$z$
\end{tabular}} & is & 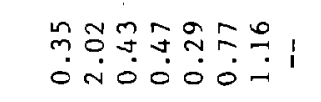 & 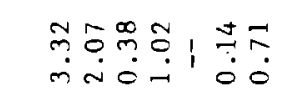 & 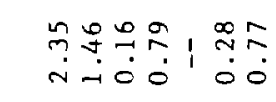 \\
\hline & & $|x|$ & 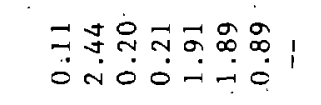 & 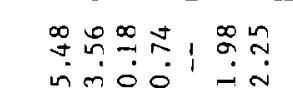 & 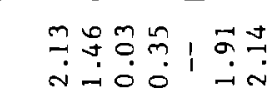 \\
\hline \multirow{3}{*}{$\begin{array}{l}\text { 옹 } \\
\text { 을 } \\
\text { 닙 }\end{array}$} & $\begin{array}{l}n \\
n \\
n\end{array}$ & $H$ & 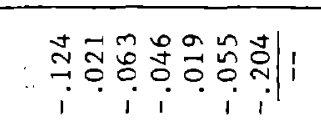 & 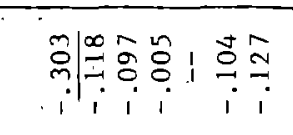 & 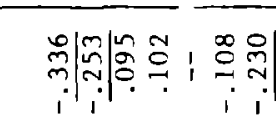 \\
\hline & \multirow{2}{*}{$\begin{array}{l}0 \\
\vdots \\
11 \\
z\end{array}$} & n & 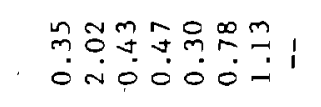 & 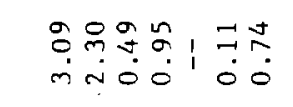 & 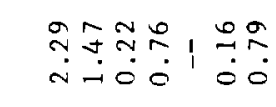 \\
\hline & & $|x|$ & 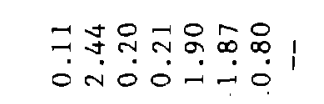 & 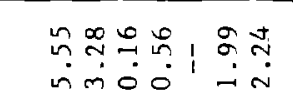 & 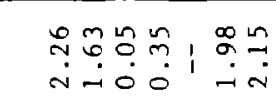 \\
\hline & \multicolumn{2}{|c|}{ 点 } & 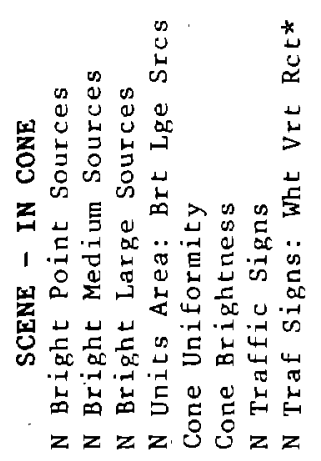 & 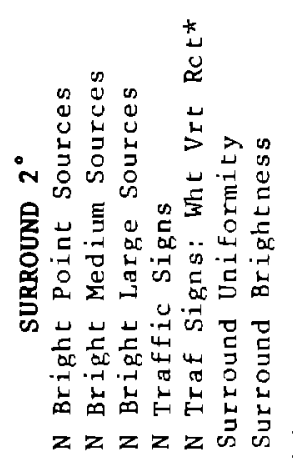 & 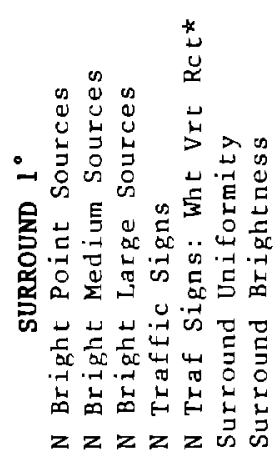 \\
\hline
\end{tabular}




\begin{tabular}{|c|c|c|c|c|c|c|}
\hline & & \begin{tabular}{|c|}
$\infty$ \\
0 \\
1 \\
$a$ \\
\end{tabular} & $H$ & 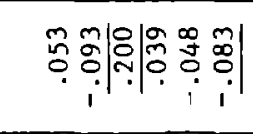 & 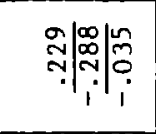 & 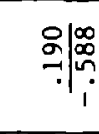 \\
\hline$\vec{H}$ & 帒 & $\begin{array}{l}0 \\
9 \\
0\end{array}$ & is & 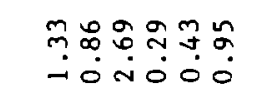 & 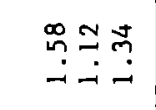 & 융 \\
\hline . & 余 & $z$ & $\mid x$ & 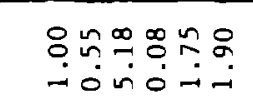 & 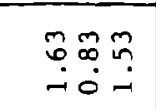 & 운 \\
\hline 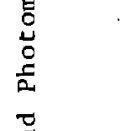 & & $\frac{n}{n}$ & H & 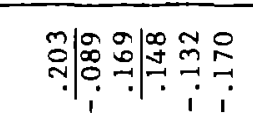 & 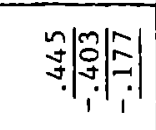 & $\stackrel{\infty}{\cdots} \mid$\begin{tabular}{l}
0 \\
\hdashline \\
0
\end{tabular} \\
\hline D্ّ & 留 & $\stackrel{0}{0}$ & D & 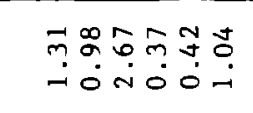 & 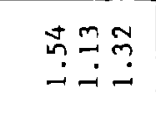 & 華先 \\
\hline 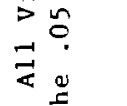 & 岌 & $z$ & $\mid x$ & 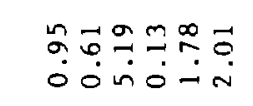 & 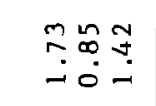 & 웅유. \\
\hline है & & $\begin{array}{ll}n \\
n \\
1 ! \\
a\end{array}$ & - & 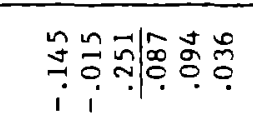 & ît: & 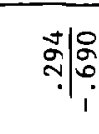 \\
\hline 岕 & 号 & $\stackrel{8}{\circ}$ & in & 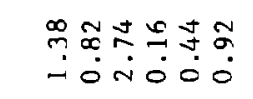 & 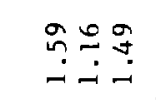 & 号志 \\
\hline$\underset{\sim}{\tilde{\pi}}$ & & $z$ & $\mid x$ & 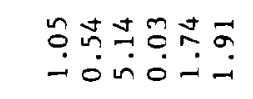 & 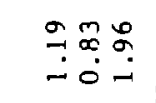 & 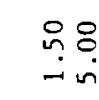 \\
\hline 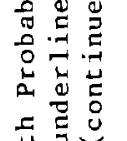 & $\underset{Z}{Z}$ & $\begin{array}{c}n \\
\sim \\
\because 1 \\
a\end{array}$ & $H$ & 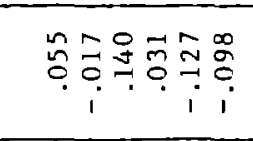 & $\bar{\imath}\left|\hat{N}_{i}\right|_{i}^{\infty}$ & : \\
\hline$\stackrel{\sim}{ }$ & $\begin{array}{l}0 \\
0 \\
0 \\
0 \\
0\end{array}$ & $\stackrel{8}{:}$ & $\infty$ & 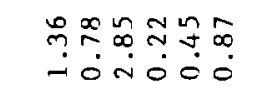 & 華足 ̃ & 号点 \\
\hline$\overline{5}$ & 宦 & $z$ & $|x|$ & 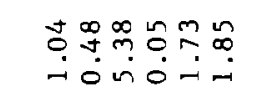 & 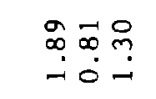 & 융ํํ \\
\hline 离 & & $\begin{array}{l}n \\
n \\
n \\
a \\
a\end{array}$ & 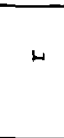 & 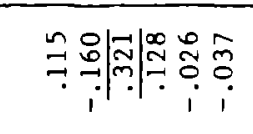 & च̂: & ㅇำ: \\
\hline$g$ & 芌 & $\stackrel{8}{0}$ & $\infty$ & 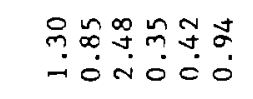 & 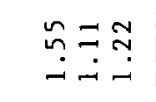 & i⿱口 犬̊ \\
\hline $\overrightarrow{0}$ & & " & $\mid x$ & $\begin{array}{l}\infty \\
\infty \\
0 \\
0\end{array}$ & 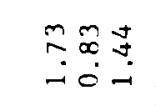 & 용 \\
\hline 吾 & & 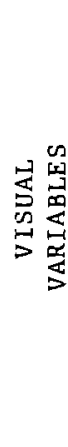 & & 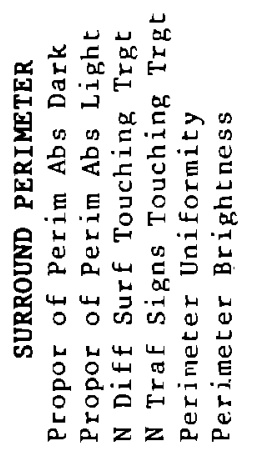 & 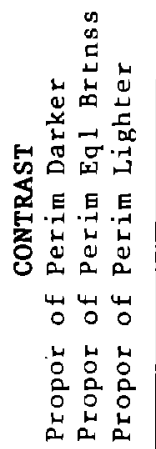 & 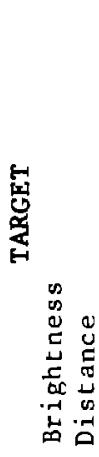 \\
\hline
\end{tabular}




\begin{tabular}{|c|c|c|c|c|c|c|c|}
\hline \multirow{3}{*}{ 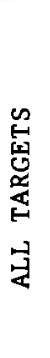 } & \begin{tabular}{|c|}
$\circ$ \\
0 \\
11 \\
$a$
\end{tabular} & $\omega$ & 羊| & â & ถึ。 & 象| & 象| \\
\hline & $\stackrel{8}{8}$ & $\infty$ & $\begin{array}{l}0 \\
\infty \\
\infty\end{array}$ & $\begin{array}{l}\dot{0} \\
\stackrel{2}{a} \\
a\end{array}$ & on & 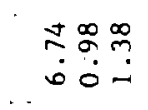 & 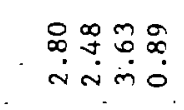 \\
\hline & 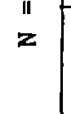 & $\mid x$ & $\stackrel{\infty}{\stackrel{\Xi}{\Xi}}$ & $\stackrel{+}{\sim}$ & 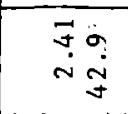 & 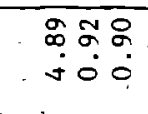 & 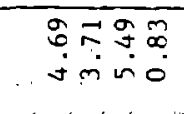 \\
\hline \multirow{3}{*}{ 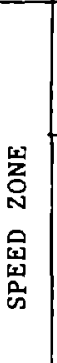 } & \begin{tabular}{l}
$\infty$ \\
$\stackrel{\infty}{a}$ \\
\hdashline \\
$a$ \\
$a$
\end{tabular} & $\omega$ & $\stackrel{\infty}{2}$ & 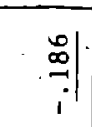 & $\begin{array}{l}0 \\
o \\
\vdots \\
i\end{array}$ & ํํำ & 品|: \\
\hline & \multirow{2}{*}{$\begin{array}{l}\stackrel{ }{\Xi} \\
11 \\
z\end{array}$} & $\sigma$ & $\stackrel{m}{N}$ & 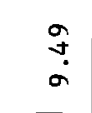 & $\stackrel{\infty}{\stackrel{\infty}{\sigma}} \stackrel{0}{\sigma}$ & 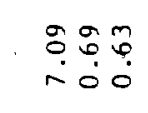 & 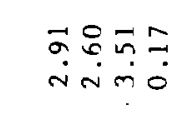 \\
\hline & & $\mid x$ & ì & $\begin{array}{l}\infty \\
\ddot{\sim}\end{array}$ & $\vec{m} \dot{n}$ & 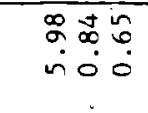 & 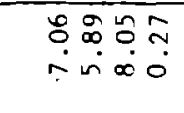 \\
\hline \multirow{3}{*}{ 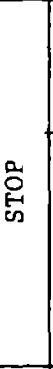 } & $\begin{array}{l}0 \\
\stackrel{\infty}{ } \\
\vdots \\
11 \\
a\end{array}$ & $\omega$ & ஜั & 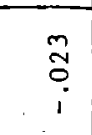 & क̊. & 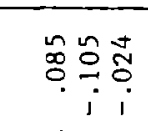 & 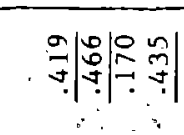 \\
\hline & \multirow{2}{*}{$\begin{array}{l}\stackrel{\bigcirc}{ত} \\
\text { "1 } \\
z\end{array}$} & $\infty$ & m. & $\stackrel{0}{0}$ & 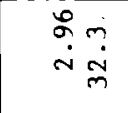 & $\vec{n} \stackrel{\infty}{\infty} 0$ & 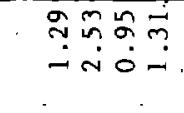 \\
\hline & & $\mid x$ & $\stackrel{a}{\vdots}$ & $\dot{m}$ & 品? & 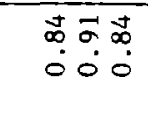 & 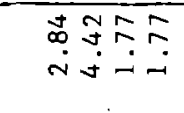 \\
\hline \multirow{3}{*}{ 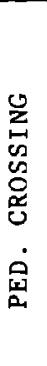 } & \begin{tabular}{|l|}
$\infty$ \\
$\stackrel{2}{-1}$ \\
11 \\
$a$
\end{tabular} & 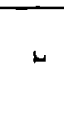 & $\begin{array}{l}\infty \\
\infty \\
i \\
i\end{array}$ & $\begin{array}{l}0 \\
\stackrel{0}{0} \\
i\end{array}$ & $\tilde{o} \tilde{\hat{q}}$ & $\hat{0} 0 \hat{0} \frac{\infty}{0} \frac{\infty}{0}$ & 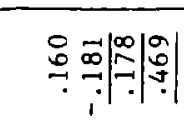 \\
\hline & \multirow{2}{*}{$\begin{array}{l}\stackrel{\bigcirc}{二} \\
\text { "I } \\
z\end{array}$} & $\infty$ & $\dot{q}$ & $\sigma$ & $\stackrel{\leftrightarrow}{\stackrel{\infty}{\sim}}$ & 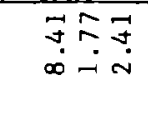 & 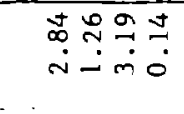 \\
\hline & & $\mid x$ & $\begin{array}{l}0 \\
\stackrel{0}{0}\end{array}$ & $\hat{\vdots}$ & 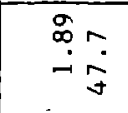 & 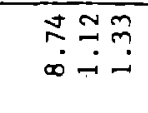 & 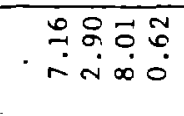 \\
\hline \multirow{3}{*}{$\begin{array}{l}\text { 号 } \\
\text { 曷 } \\
\text { 䓢 }\end{array}$} & $\stackrel{\infty}{2}$ & - & 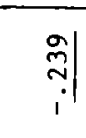 & 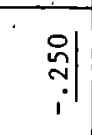 & $\begin{array}{cc}0 \\
8 \\
0 & 0 \\
1 & 1\end{array}$ & ?ొ: & 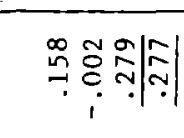 \\
\hline & \multirow{2}{*}{$\begin{array}{l}\stackrel{ }{\mathrm{N}} \\
\text { "I } \\
z\end{array}$} & $\infty$ & ن. & $\dddot{\sim}$ & 吕 & 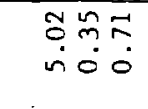 & 㞧 \\
\hline & & $\mid x$ & $\begin{array}{l}\circ \\
\dot{\theta}\end{array}$ & $\stackrel{+}{\sim}$ & \begin{tabular}{l}
$\dot{0} 0$ \\
$\dot{0}$ \\
$\dot{v}$ \\
\multirow{v}{*}{}
\end{tabular} & $\begin{array}{l}a \infty n \\
\because 0: 0\end{array}$ & 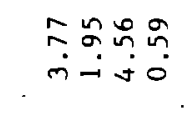 \\
\hline & 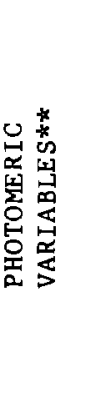 & & 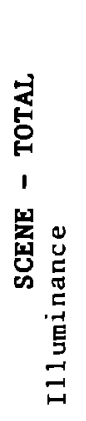 & 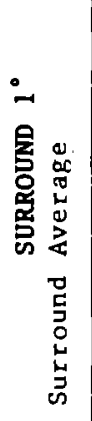 & 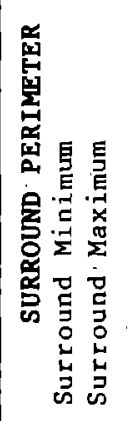 & 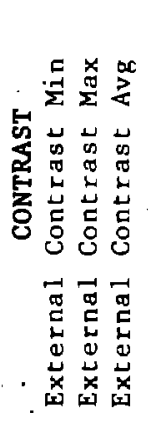 & 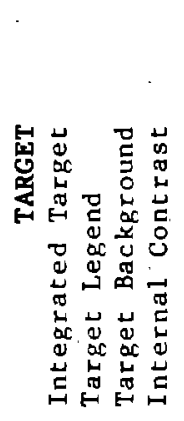 \\
\hline
\end{tabular}




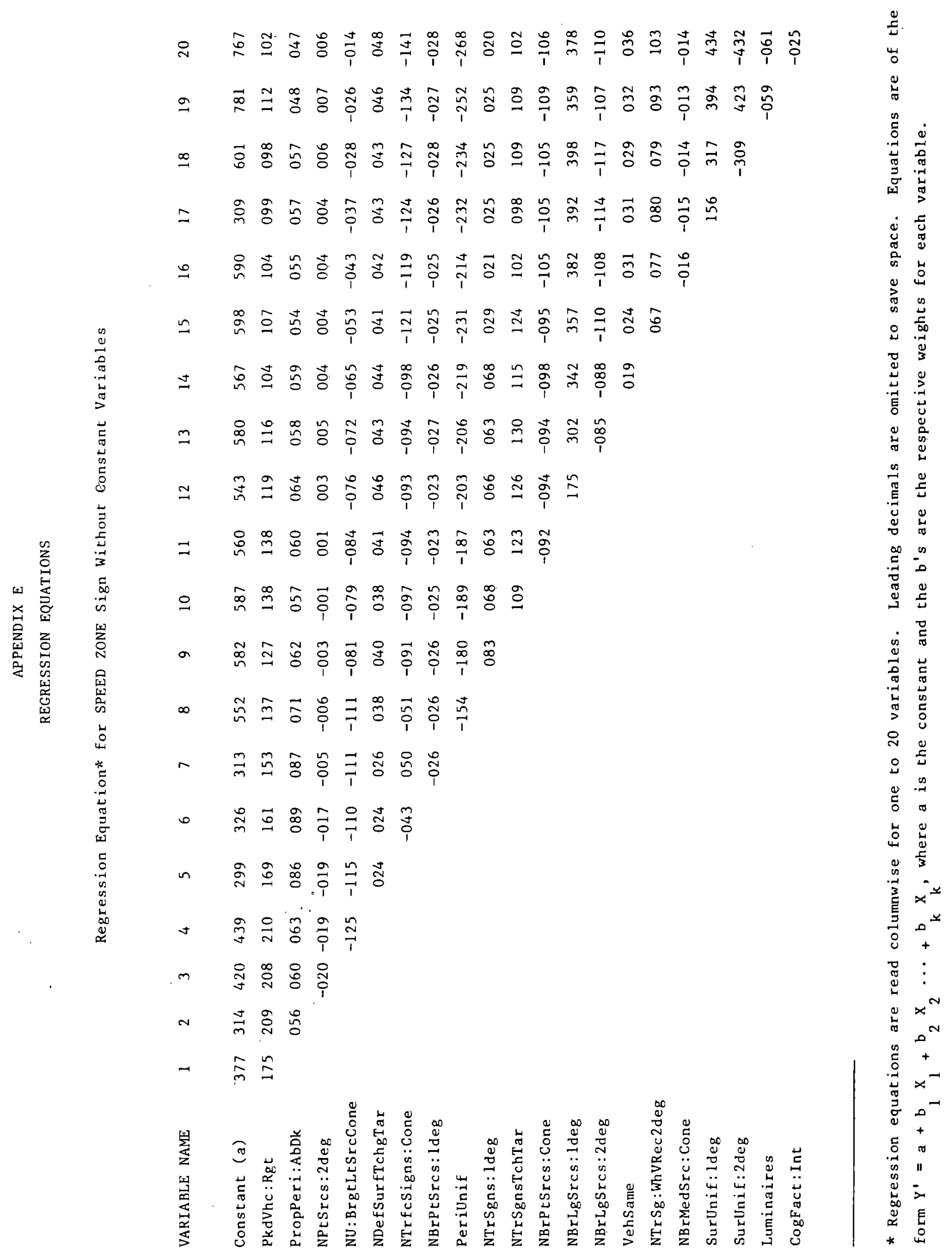




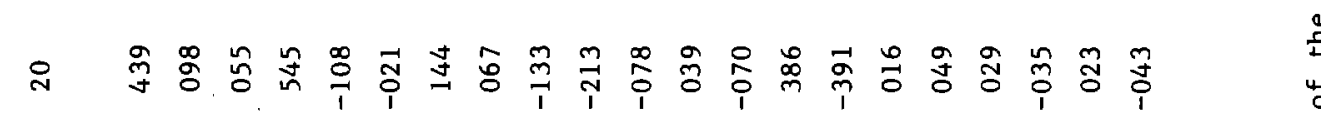

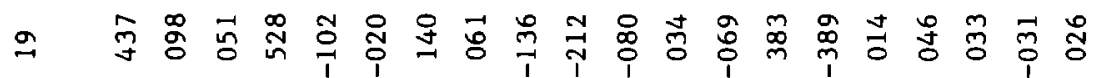

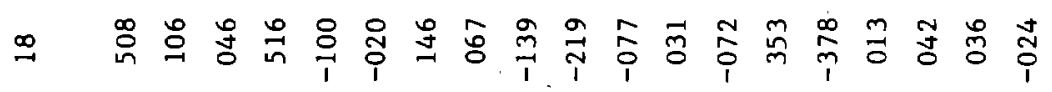

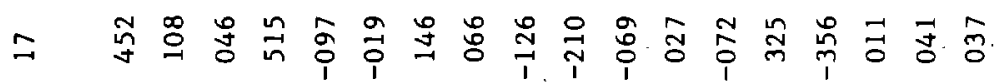

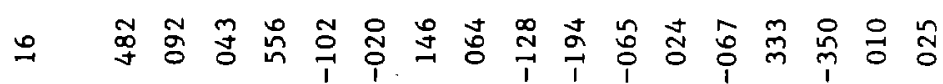

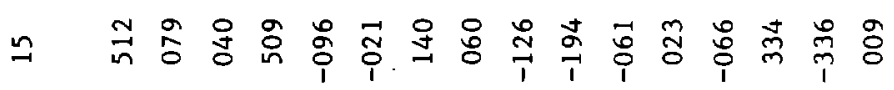

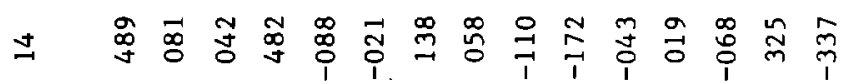

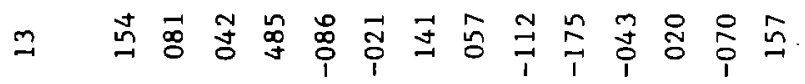

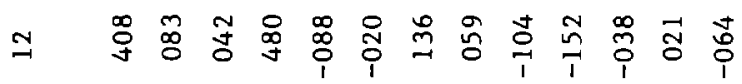

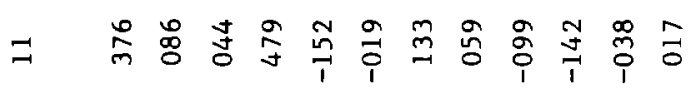

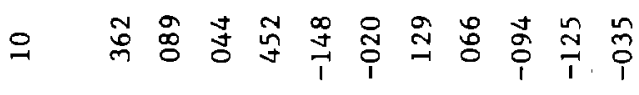

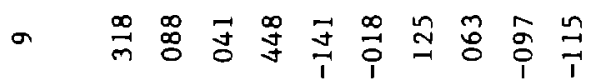

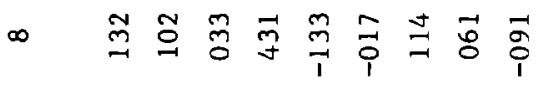

一

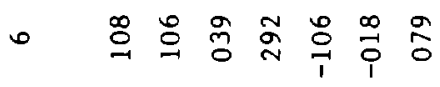

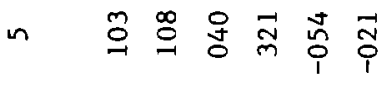

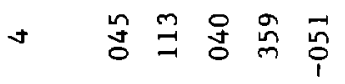

-

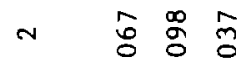

-

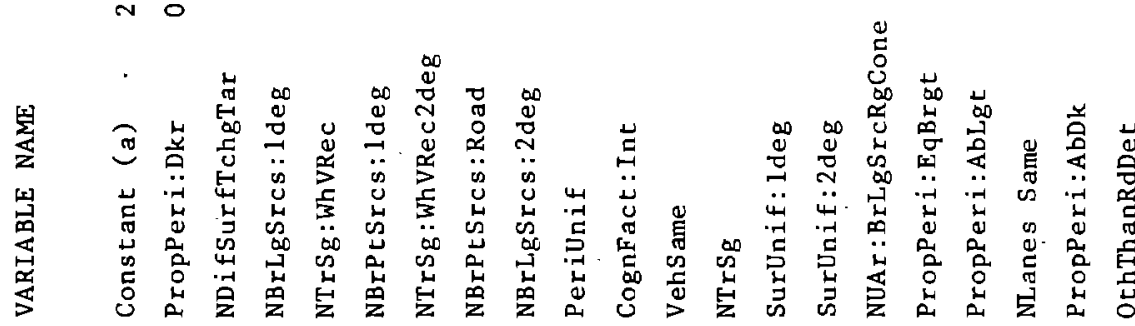




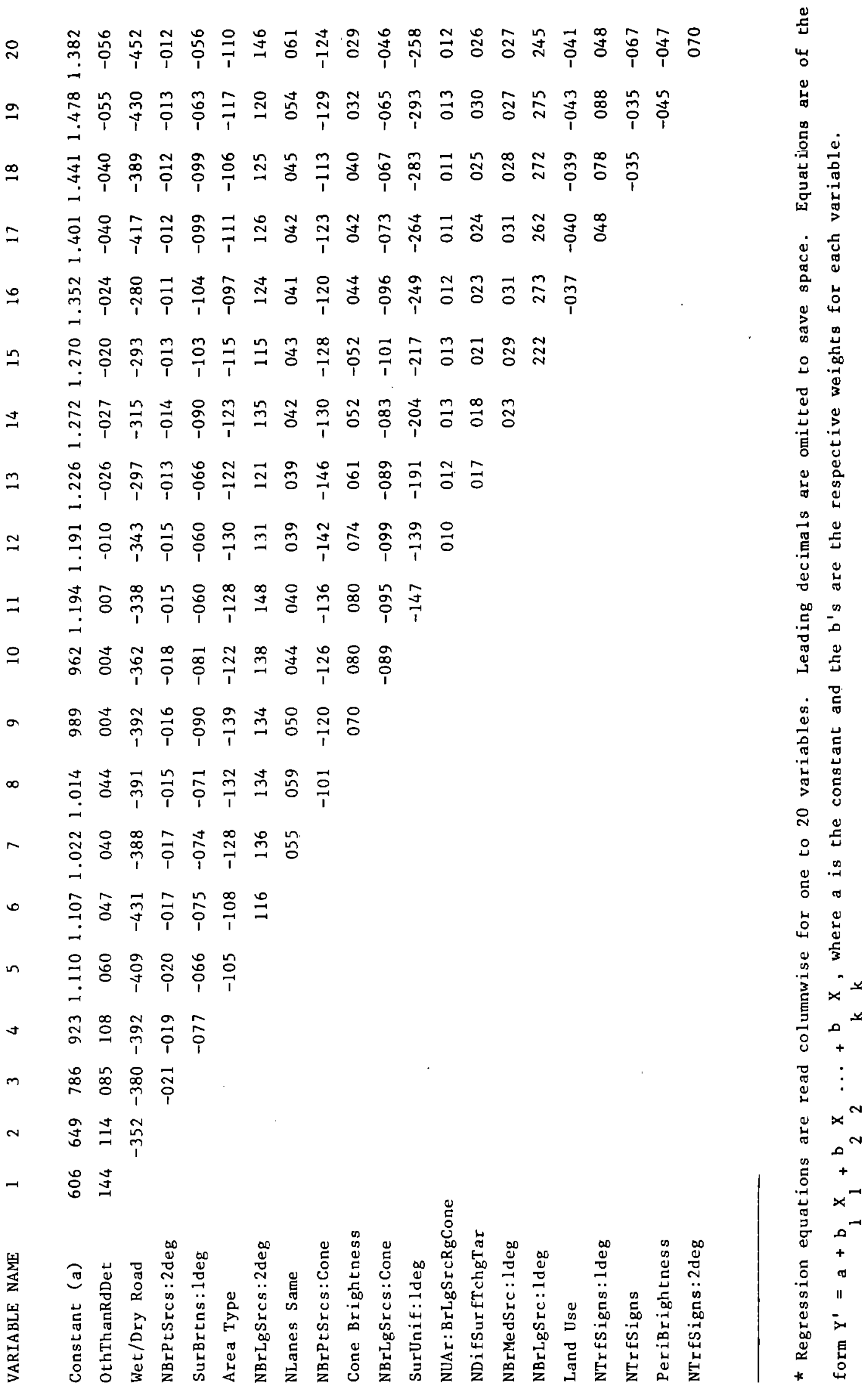




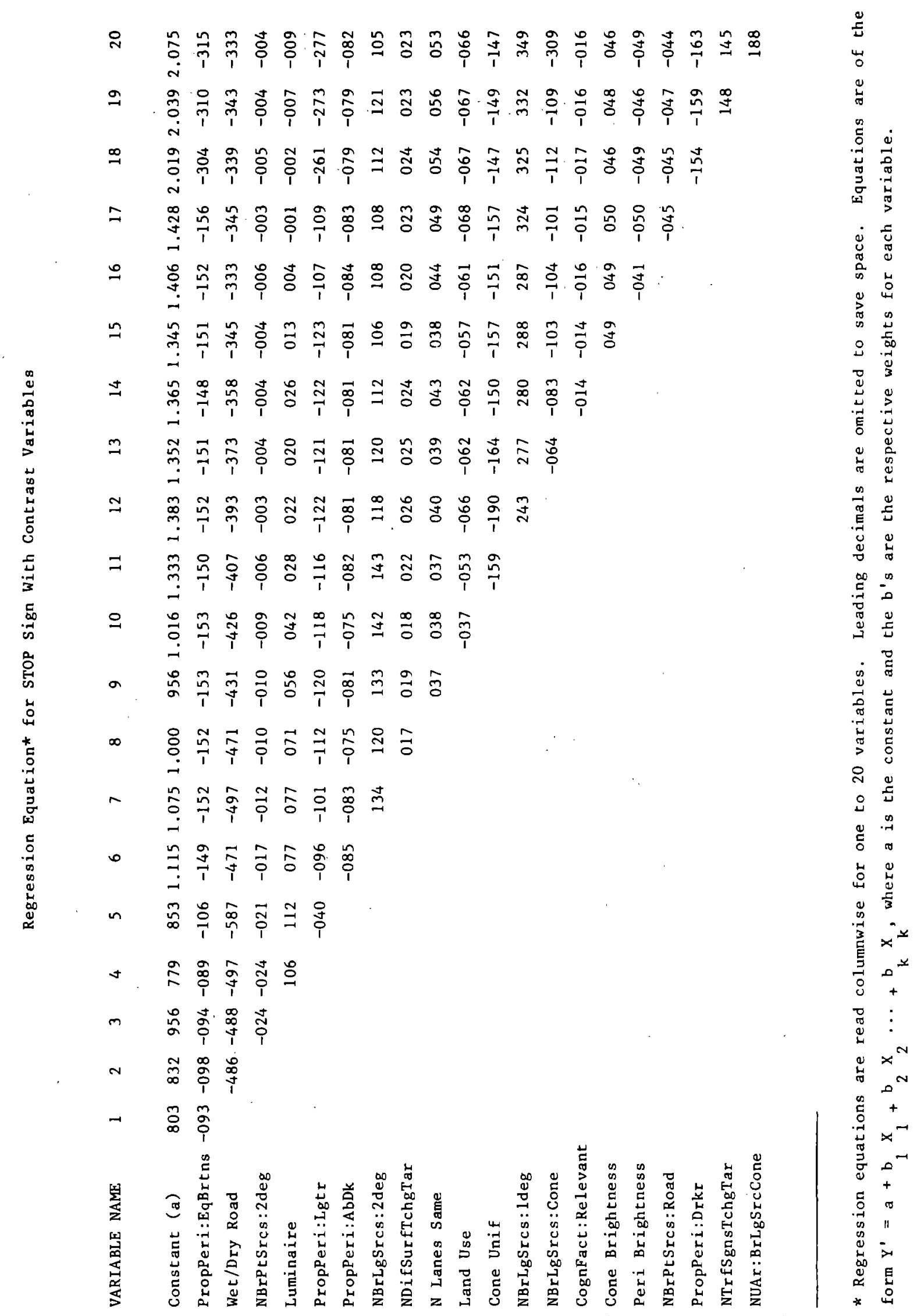




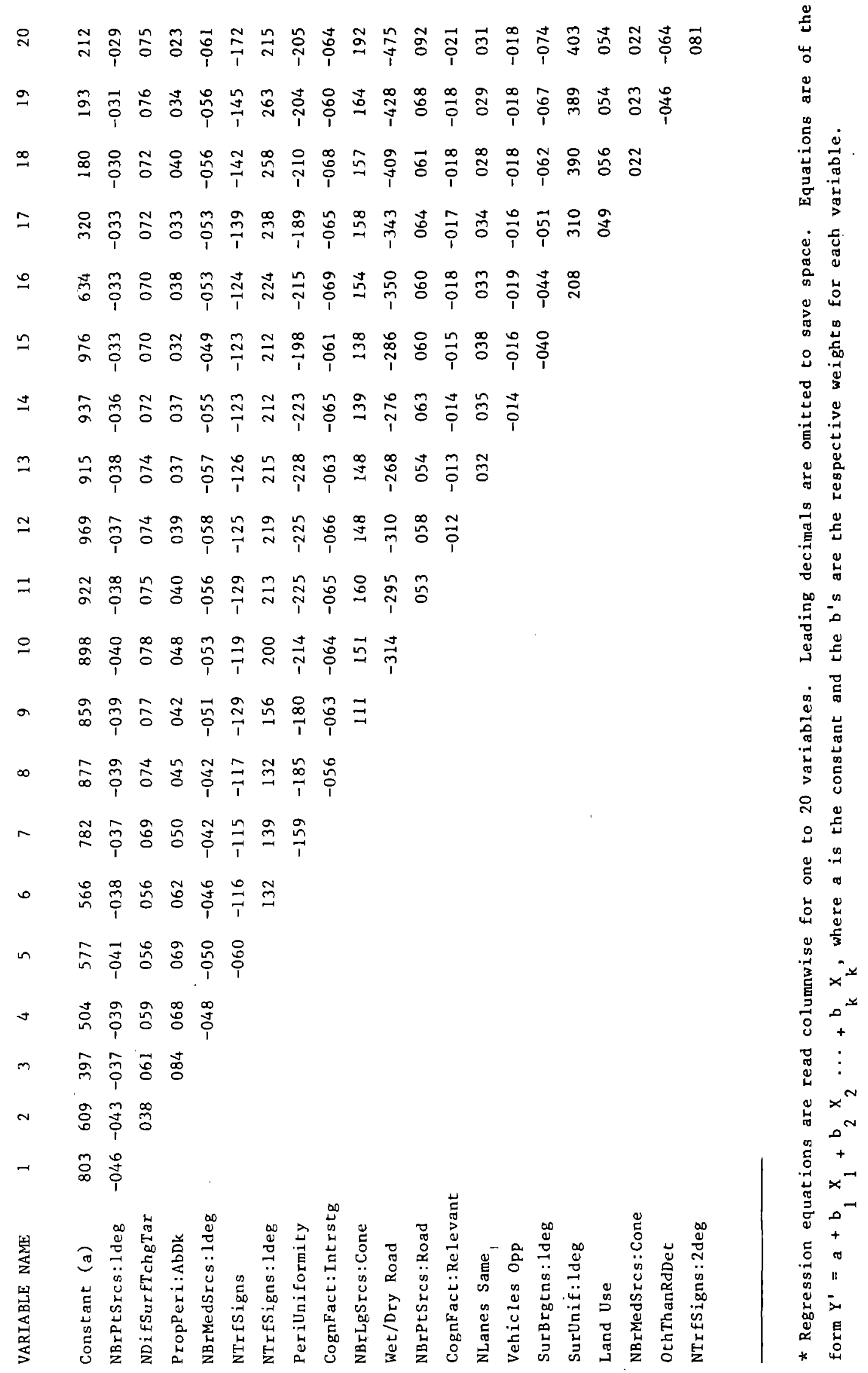




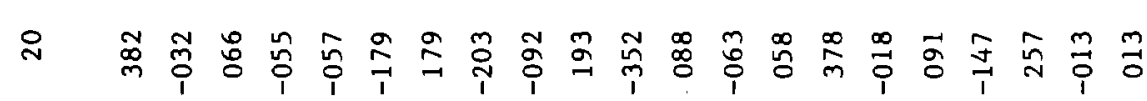

$\stackrel{\Xi}{ \pm}$

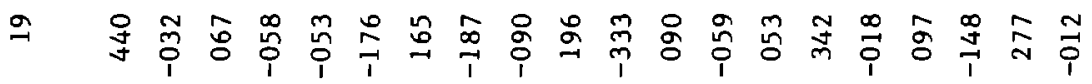

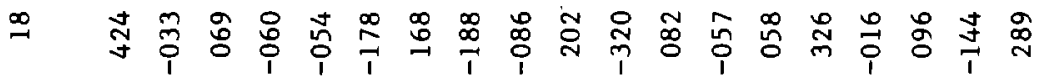

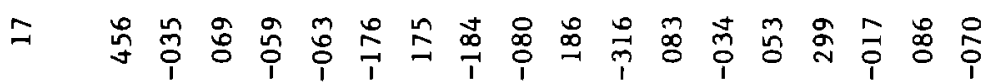

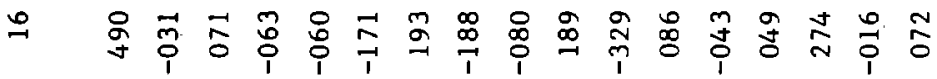

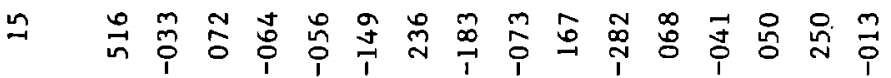

-

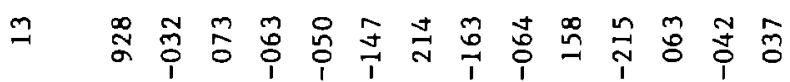

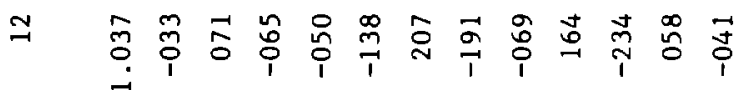

$\Rightarrow \quad$ 웅

융

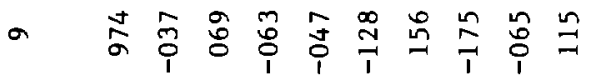

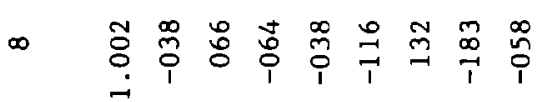

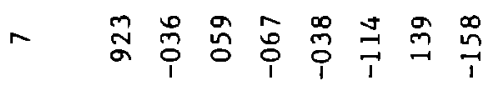

- $\quad \vec{\ddagger} \hat{i}$ 寸

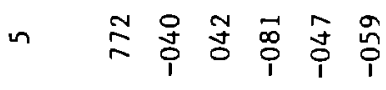

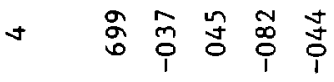

m J 寸

$\sim \quad$ \&

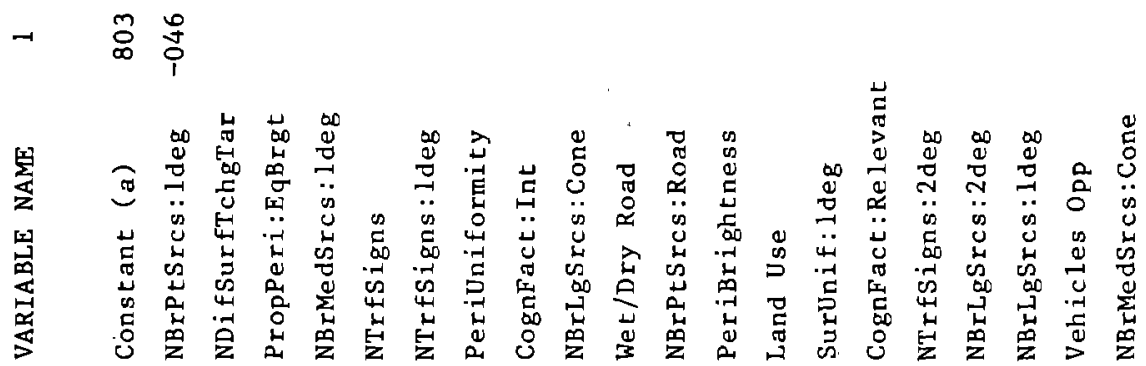




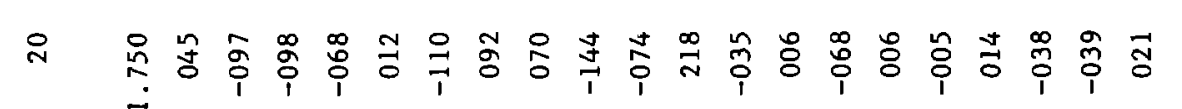

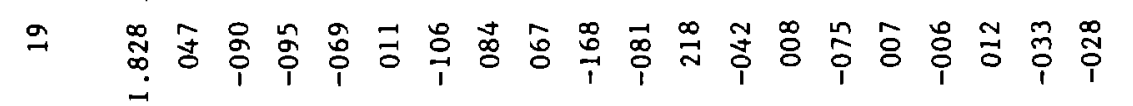

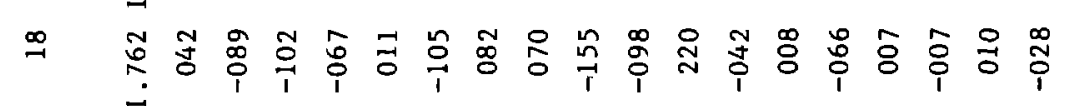

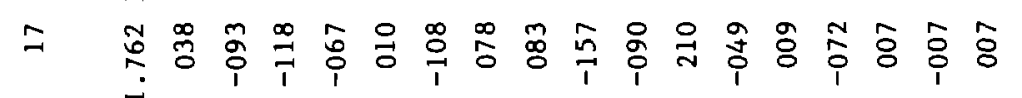

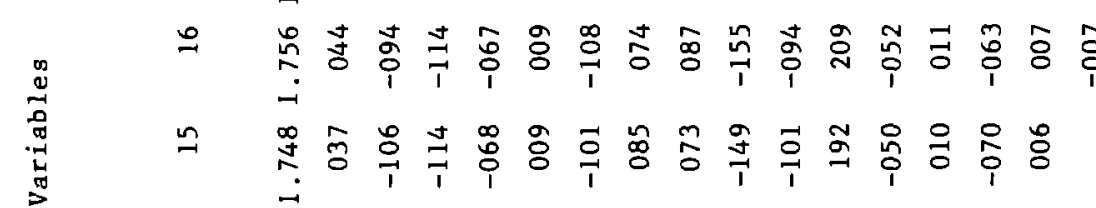

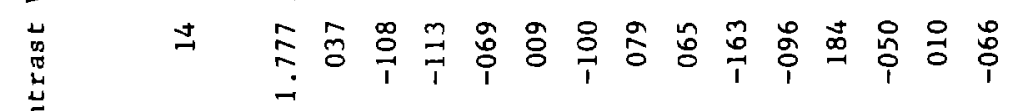

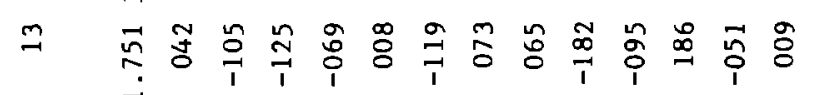

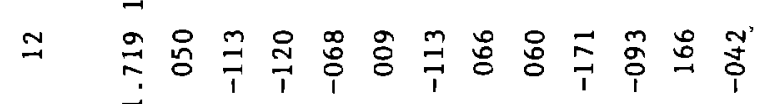

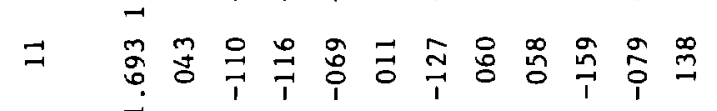

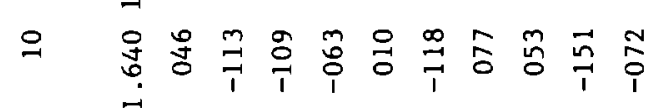

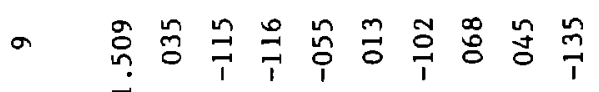

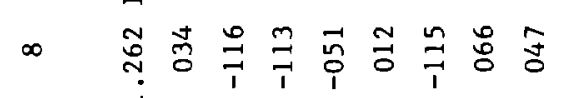

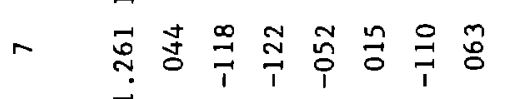

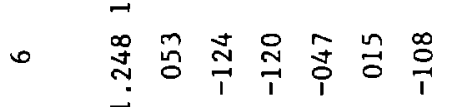

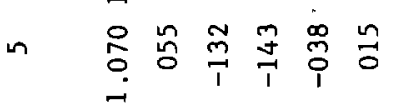

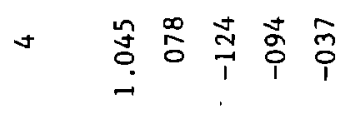

-

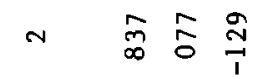

- 苗

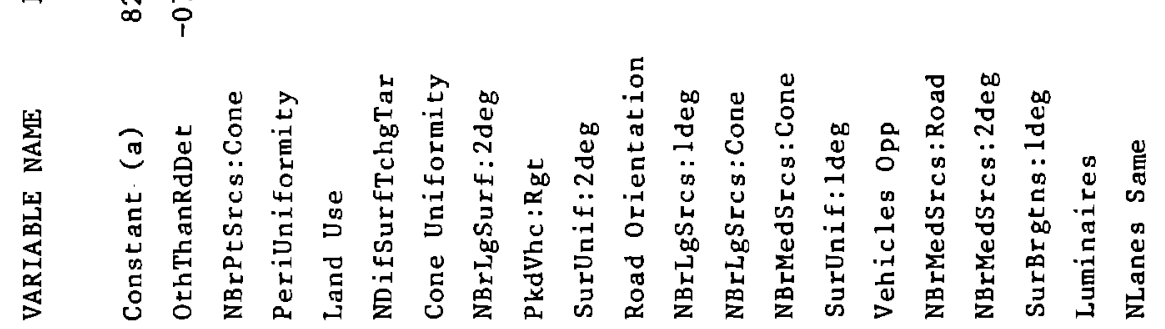

$\stackrel{0}{ \pm}$

ปั

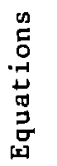




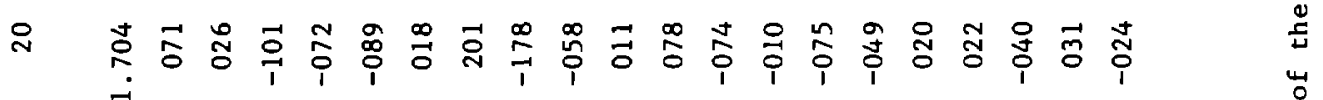

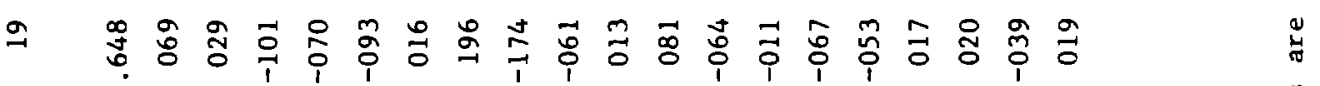

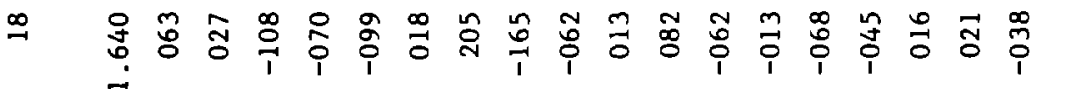

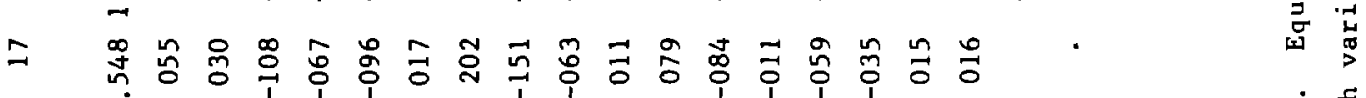

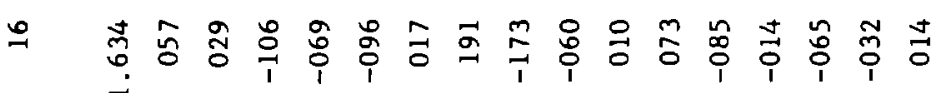

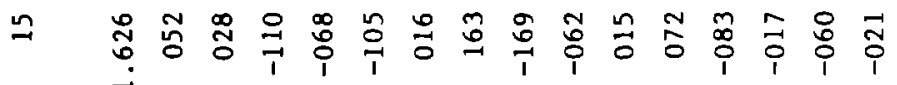

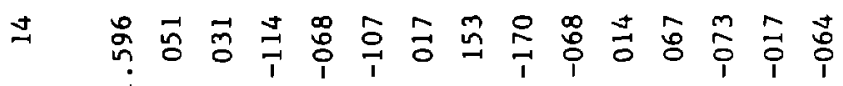

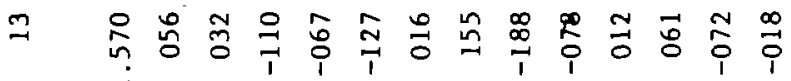

$$
\begin{aligned}
& \approx \quad \stackrel{0}{\circ} \text { す }
\end{aligned}
$$

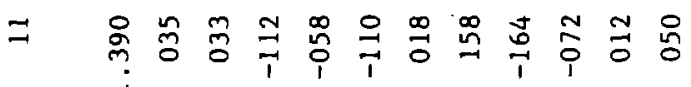

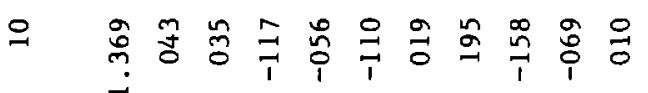

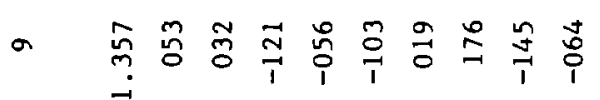

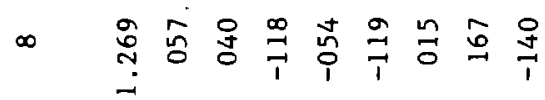

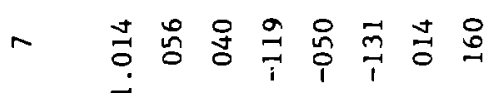

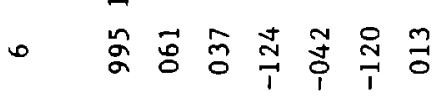

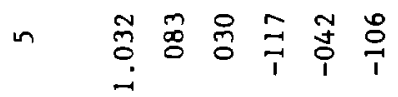

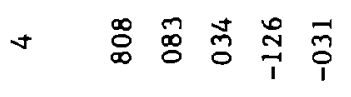

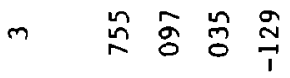

$$
\begin{aligned}
& \text { N 疍骂 } \\
& \text { - } \quad \infty_{0}^{N}
\end{aligned}
$$

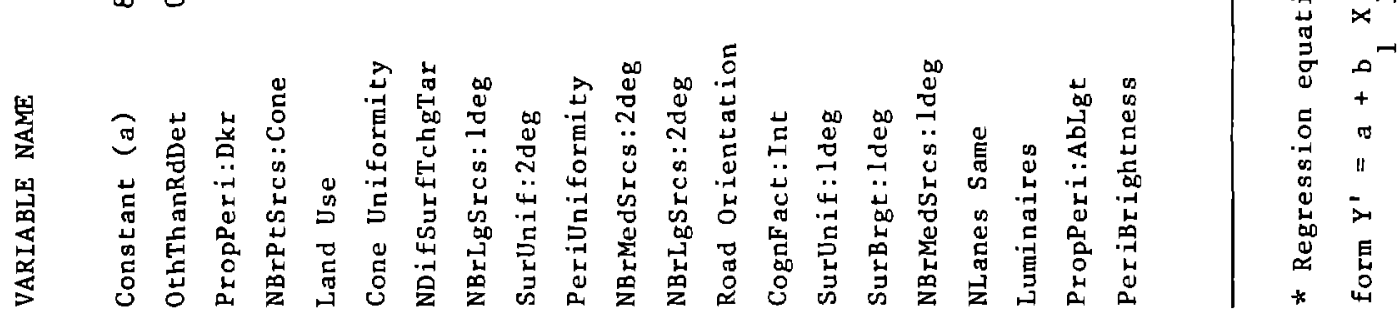




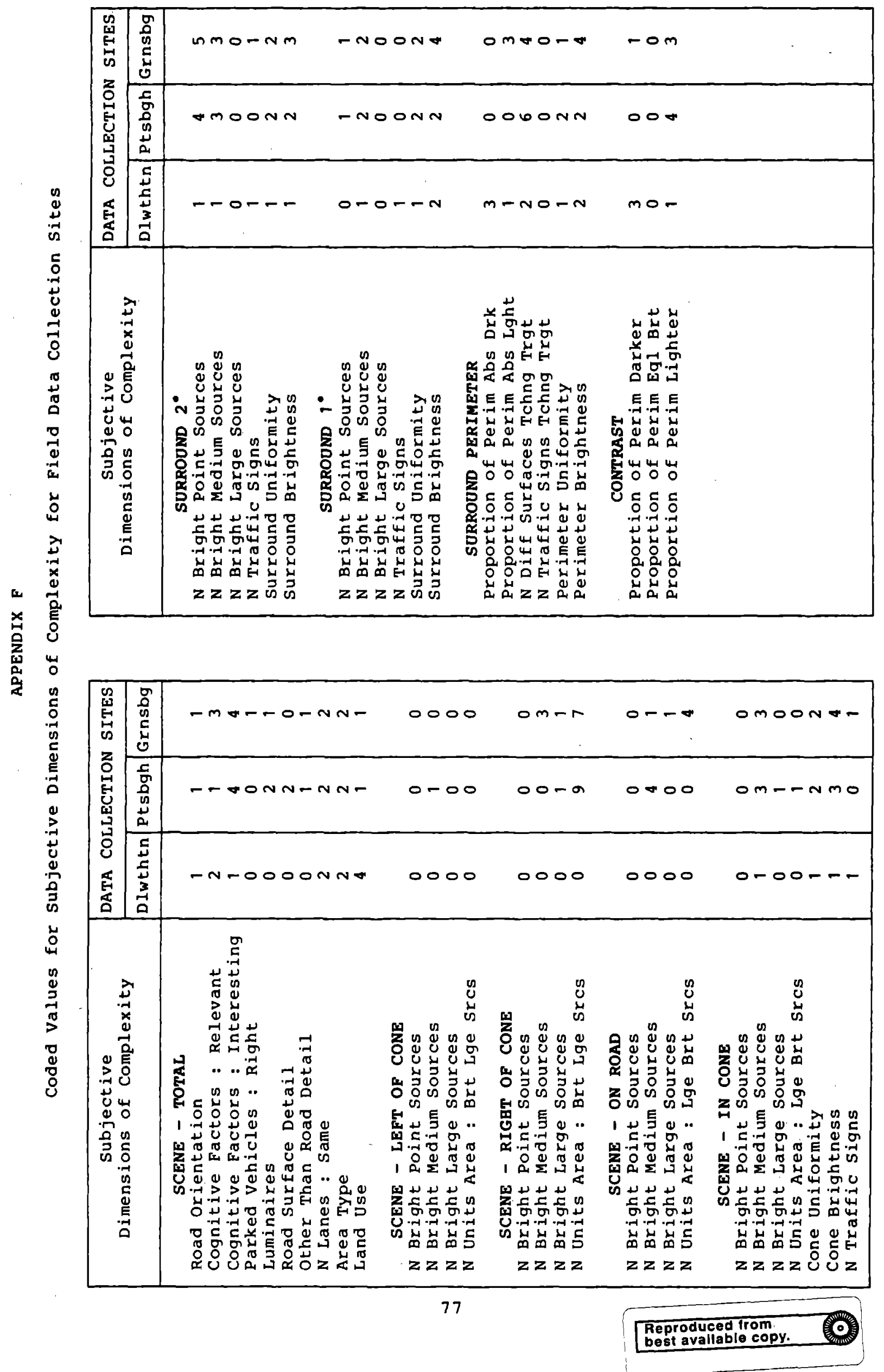


